SERVIÇO DE PÓS-GRADUAÇÃC DO ICMC-USP Data de Depósito: 17.06.2005 Assinatura: lowardeceleri (

\title{
Modelos estocásticos contínuos e discretos aplicados em finanças
}

\section{Juliana Cobre}

Orientador: Prof. Dr. Marinho Gomes de Andrade Filho

Dissertação apresentada ao Instituto de Ciências Matemáticas e de Computação - ICMC-USP, como parte dos requisitos para obtenção do título de Mestre em Ciências - Área: Ciências de Computação e Matemática Computacional.

USP - São Carlos

Junho/2005 
A meus queridos pais. 


\section{Agradecimentos}

Agradego a Dents e ao men anjo da guarda peta saúle o por todos os insightir. Por teren colocaklo m mou caminho pessoas maravilhosas, que diretamente on indirotamente me ajulatram muito.

Agradego especialnuente nos mens pris, João e Edua, que scmpre me deram incentivo en seguir mens estudos e muito conforto para cumpri-los. Sän fontes de forças na minlad huta an sempre dar o molhor de minn. Fxemplos de honestidade e resperito.

Agralego as arnigas que moraram comigo, Carla Bossu, Juliana B. Gabrido, Karina K. de Lima, Priscila Ap. do Momes e Viviane S. Tonali, que ne suportanan nos monentos de: crise, gue nue deram apoio o que me divertivan muto

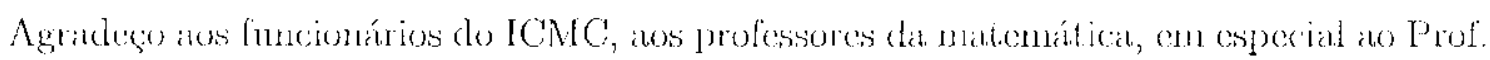

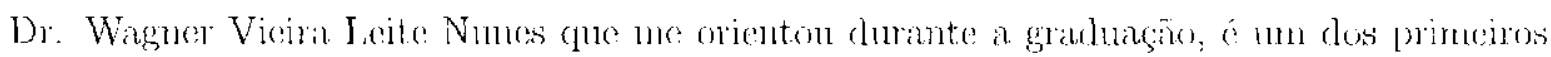

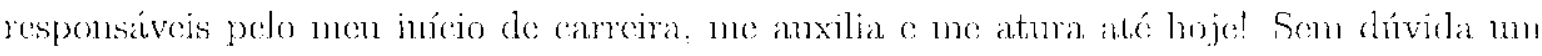
gramle anigo.

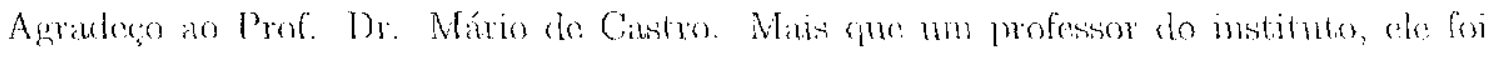
jara mim um colega de laboratório, um solucionador de mutos dos mens problemas com o rompntaralor e com os gráficos, um incentivador da pesquisa. Sempre aprendi rmito com ele, nuesmo nats couversatis matis descontrajdas, fora dos projetos científicos. Uma pessoil uluniurivel

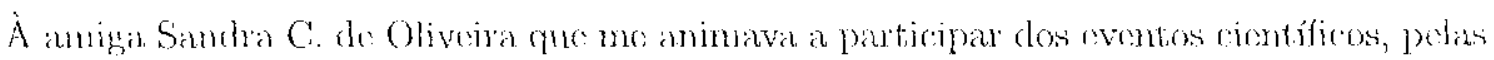
dicas de sites, nateriais e dia abordagem dos resultados. Por tanto ter onvido meus desábafos nos momentos dilíceis. E também pelos bons mumentus de descombaño. Agradego ì Vera L. D. Tomazella pelo matrerial (pue me fornecen e por ser läo prestativa.

'Lambém agradego muito a todo o pessonal do kaboratório de estatistica, que sempre colitburou mas minhas dúvidias.

F: finalumente agradero a men orientador, Prof. Dr. Mininho G. de Audrade Filho, pelo projeto e pelos monentos dedicaklos a ele. 


\section{Resumo}

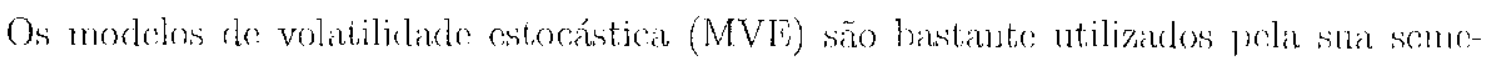
Thançan com os modelos habitualrnente usados na Teoria Financeira. Nos MVE a volatilitilade inulepende dos retornos passados e é modelada como ma variável latente não obscrvada, atrivés de unna conponente predilível e outra alcatónia. A função de verossimibhança desses

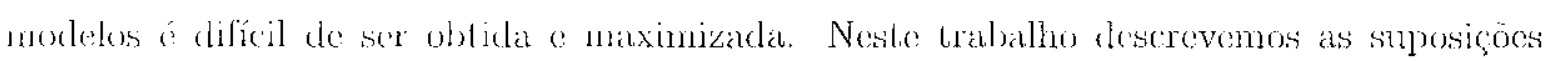

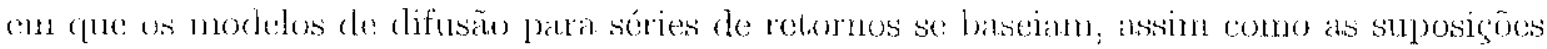
tomnadas pola modeligem discreta. Apresentamos os MVF e alguns de scus métodes de es-

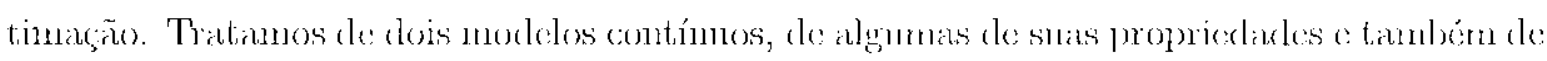

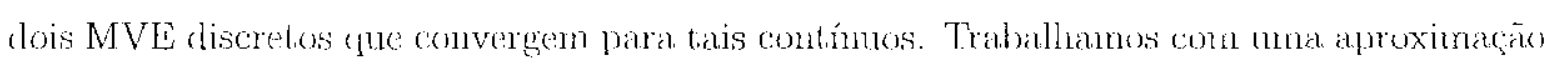
linear de um keles, apresentanulo o filtro de Kalman, e sua verossimilhauça ubtida depois da filtragem. () algorituno de Metropolis-llastings foi cmpregato na aborelagem dir verossimi-

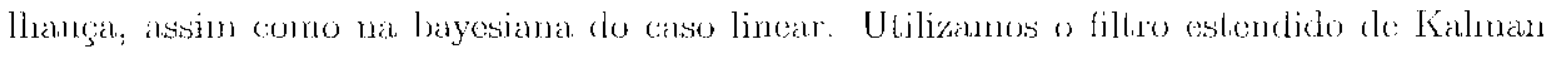

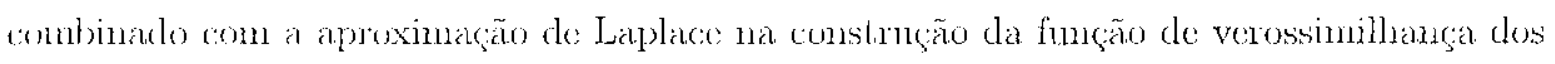
dois MVE abordandos neste trablallo. 


\begin{abstract}
The storhastic volatility models (SVM) are cuite habitulally used by their similarity with the morels used in the Financial Theory. In them the volatility is described through their last values and it does not depend on the last returns. The likelihood function of SV is difficult of being obtained and maximized. In this paper, we have described the lypothesis in which the diffusion modelo for series of retums are based, as well as the suppowitions takien by the discrete norlelling. Wo presented SVM and some of their estimate inetlods. Wo treated of two (ontinnous models, of some of their properties and also of two discrete SVM flate converge for the continnous ones. We worked with a linear approach of one of theme presenting the Kalman filter, and its likelihood obtaned after the fill ration. The Metropolis-Hastings algorithm was used in the approach of the likelihood, as well as in the Bayesian of the linear case. We used the extended Kalnan filter combined with the Laplace approxination in the construction of the likelihood function of the two SVMI approached in this work.
\end{abstract}


1 Introdução 1

1.1 Terminologia m Finangas . . . . . . . . . . . . . . . 3

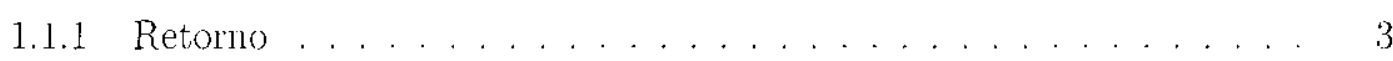

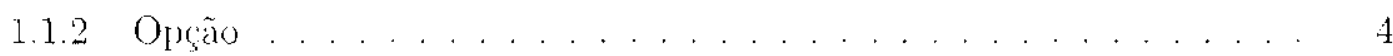

1.1.3 Volatilidade em Mercados Financeiros . . . . . . . . . . . . 5 5

1.2 Dinânica do Pręo do Ativo . . . . . . . . . . . . . . . . 6

1.2.1 Modelos Discretos. . . . . . . . . . . . . . . . . . . . . . . 8

1.3 Vodelos de Volatilidade Estocástica . . . . . . . . . . . . . . . . 9

1.4 Mlétodus de Estintaçălo . . . . . . . . . . . . . . . . . . . 11

2 Modelos I e II 13

2.1 Prelininares . . . . . . . . . . . . . . . . . . . . . . 13

2.2 Modelo discreto por modelo de difusão $\ldots \ldots \ldots \ldots$

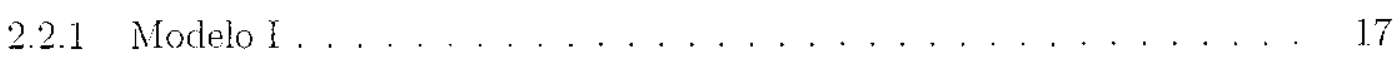

2.2 .2 Modelo II . . . . . . . . . . . . . . . . . . . . . . . . . . . . . 20

2.3 Conlucendo os Modelos . . . . . . . . . . . . . . . . . . . . . 22

2.3 .1 Modclo I . . . . . . . . . . . . . . . . . . . . . . . . 24

2.3 .2 Modelo II . . . . . . . . . . . . . . . . . . . . . . 25

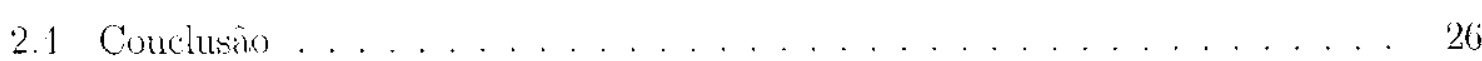


3 Modelo Lincar $\quad 27$

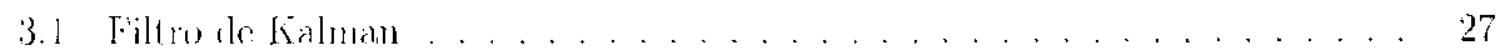

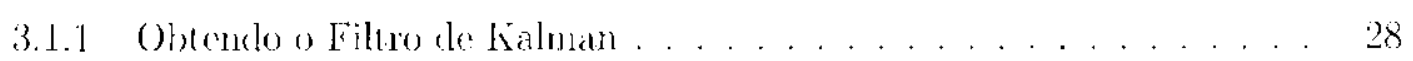

3.2 Nodelo Invariante no $\mathrm{Tempo} \ldots \ldots \ldots \ldots \ldots$

3.2 .1 Verossinilhanca . . . . . . . . . . . . . . . . . . . 33

3.2 .2 Mbordagem Bayesiana . . . . . . . . . . . . . . . 36

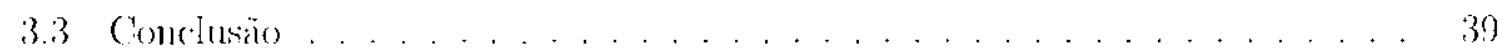

4 Inferência para o Modelo I $\quad 41$

t.1 Filtro Fstondido de Kalnan ..................... 11

1.2 Nodelos Condicionalunente Gaussianos . . . . . . . . . . . . 13

$1.3 \quad \mathrm{MVFH} \ldots \ldots \ldots \ldots \ldots \ldots$

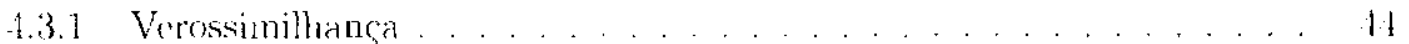

1.1 Abordagen Bayesiana . . . . . . . . . . . . . . . 47

1.j Molelo I . . . . . . . . . . . . . . . . . . . . . 18

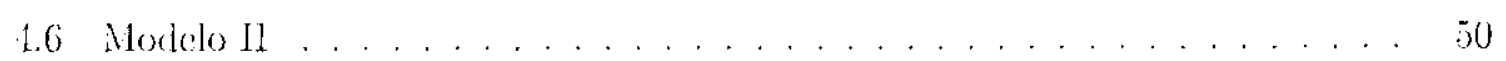

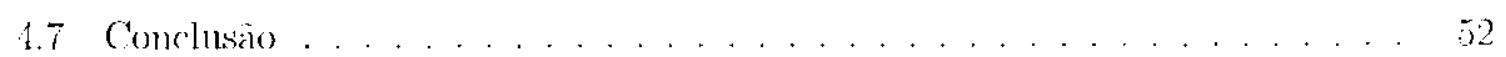

5 Resultados $\quad 53$

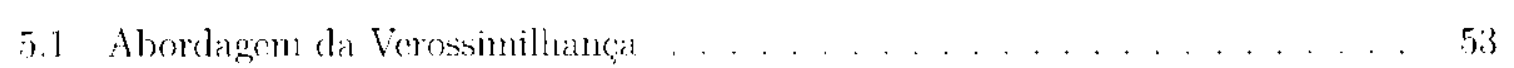

5.2 Abordagem Bayesiana . . . . . . . . . . . . . . . . . . . . . . . . .

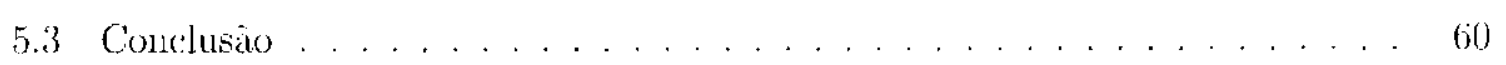

A Métodos Numéricos

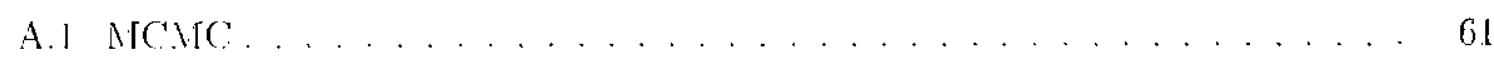

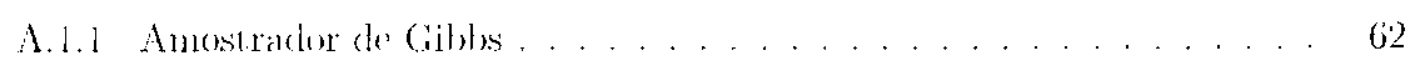

A.1.2 Motropolis-Hastings . . . . . . . . . . . . . . 62

1.2 Aproximagäo de Laplace . . . . . . . . . . . . . . . . . 63

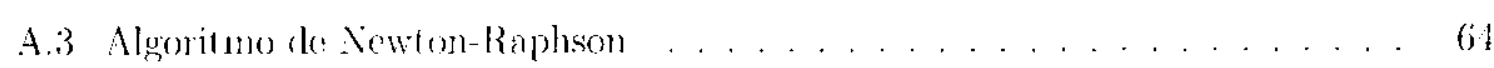

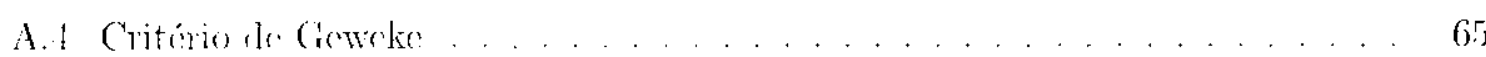

$\begin{array}{lr}\text { B Modelos Discretos } & 67\end{array}$

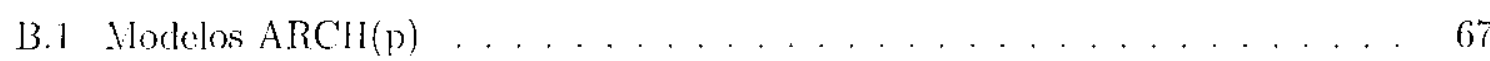




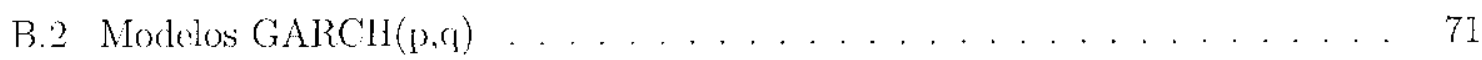

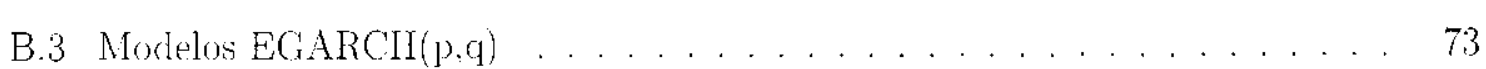

B.4 Modelos ARCH-M . . . . . . . . . . . . . . . . . 73

B.5 Modelos IGARCHI . . . . . . . . . . . . . . . . . 71

$\begin{array}{lr}\text { Referências Bibliográficas } & 79\end{array}$ 


\section{Lista de Figuras}

1.1 Sćrie de retomo e série de log-retomo da Telebras $(02 / 01 / 92$ a $05 / 01 / 96) \ldots$. A

5.1 Histogramiss das mostras solecionadas: MLT A. . . . . . . . . . 55

5.2 Gráficos dass amostrats selecionadas: MIT A. . . . . . . . . . 5i

5.3 Hintogramas das amostras solccinnadas: MIT B. . . . . . . . . 56

5.1 Grálicos das amostras sclecionadas: MIT B . . . . . . . . . . 56

5.5 Histograndis das amostras selecionadas: MIT A bayesiano. . . . . . . . . 58

5.6 Gráficos das annostras selecionadas: MIT A bayesiano. . . . . . . . . . 58

5.7 Histogranis das anostras selecionadas: MIT B baycsiano. . . . . . . . 59

5.8 Grúfices dias annostras selecionadas: MIT B bayewiann. . . . . . . . . 59 


\section{Lista de Tabelas}

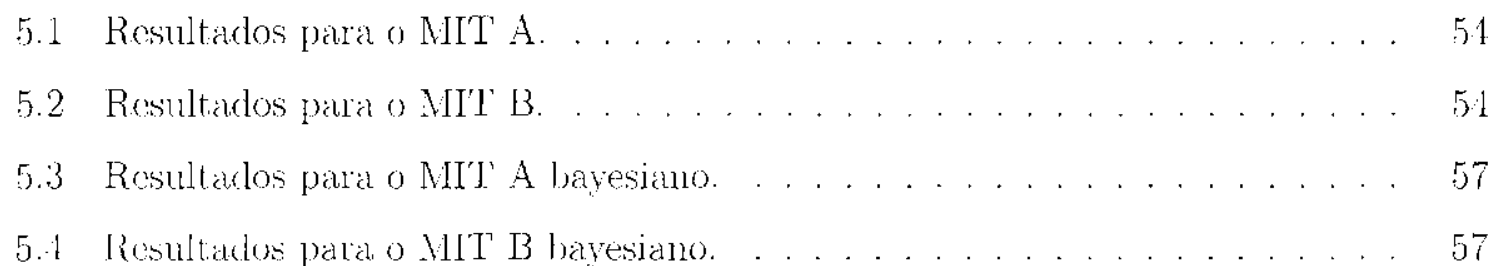




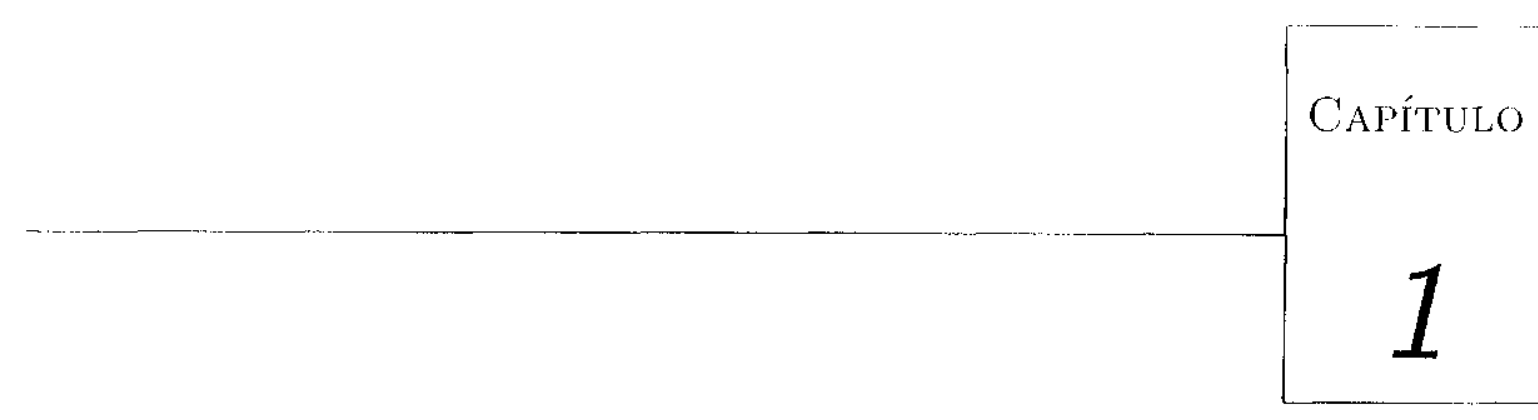

Introdução

Os prineiros estudos dedicados a descrever o conportanento do retomo on log-retorno

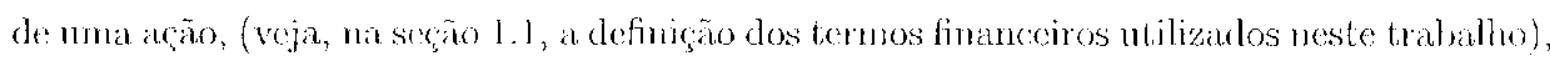
enfocavam apenas o prineiro momento do nodelo. Moneutus de orden mais alta, conno por exemplo a variancia, eram ignorados pois eran considerados constantes. No entanto, na prática, into nào ocorre. Un exemplo simples alesse fato é notar que cun dias com nator

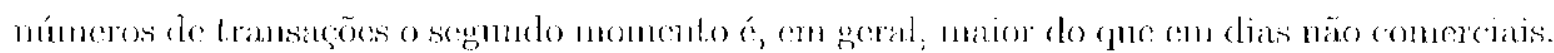

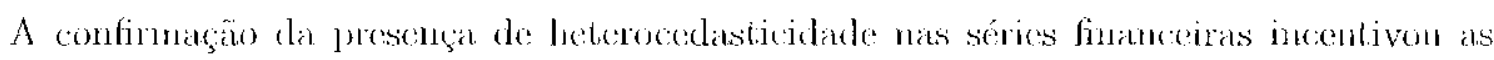

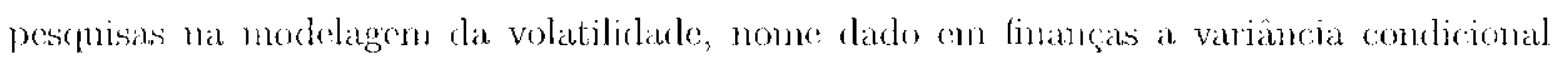

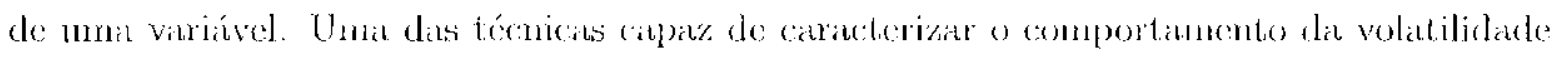
do retorno toi introduzida por Fuggle (1982) e é chamada de processo anto-regressivo com heterocedasticidarle, ou simplesmente ARCII. São modelos a tempo discreto fundancntados

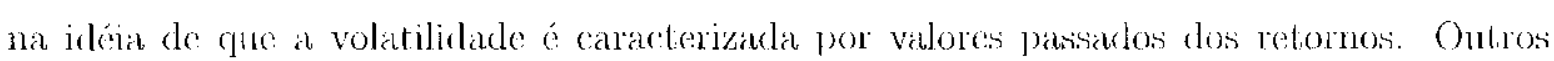
modelos discretos foran desenvolvidos a partir do ARCH, como por exemplo GARCH proposto por Bollerslev (1986), LGARCH apresentado por Velson (1991), IGARCH por Eingle \& Bollesslev (1986), cutre onluos (voja pex. Hanilton, 1994). Una ampla revisato das puo- 
pricrades desses modelos pode ser encontrada em Pollerslev, Chou \& Kronor (1992) e una

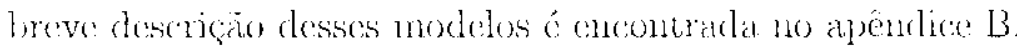

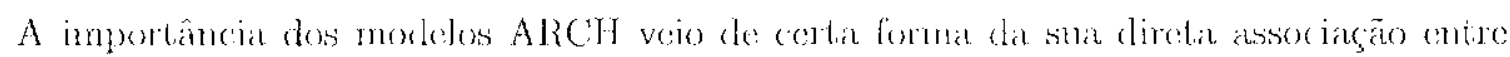
vaniância e risco e da fundannental relagio contre risco e retomo (no comércio de compra).

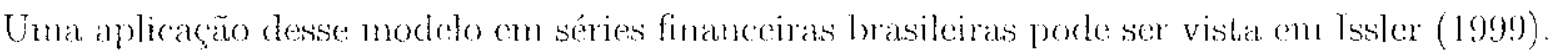

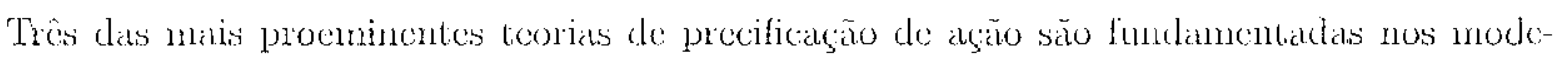
los AliCH: Cayital Asst. Pricung Model (CAPM), Consumption-Based CAPM o Arbitray Pricing Theory (AP'L).

Existem também os mokelos de volatilidade estocástica (MVE) propostos originalmente jor Taylor (1982). A fundamental diferença entre osses modelos o os modelos ro tipo ARCH

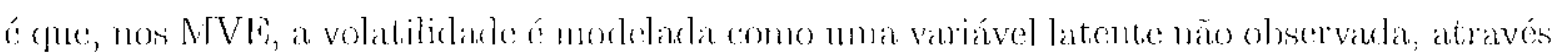

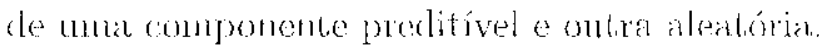

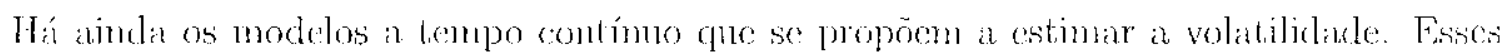

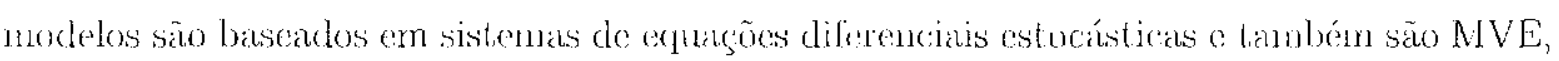

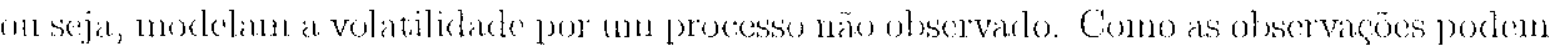
ser feilas apenas a tempo discreto, existo un grande interesse em ajoroxima um modelo do

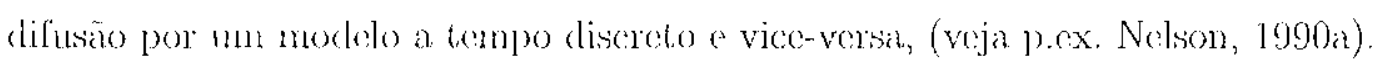

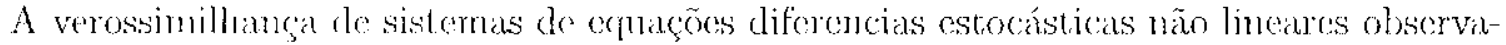

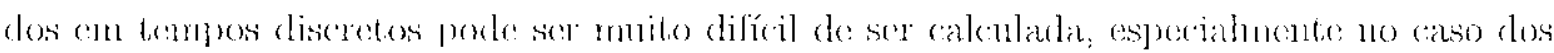

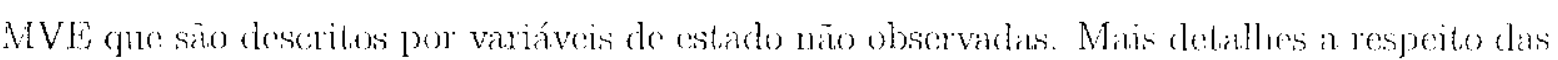
difionldades e das vantagens deste tipo do modelo serão dados labs seroes 1.3 e 1.4 .

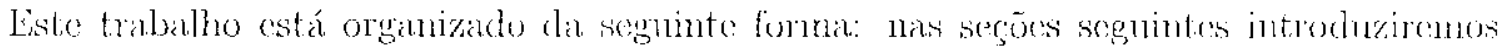
algunis termos financoiros necessários para sen entendimento, apresentaremos as suposiçoes

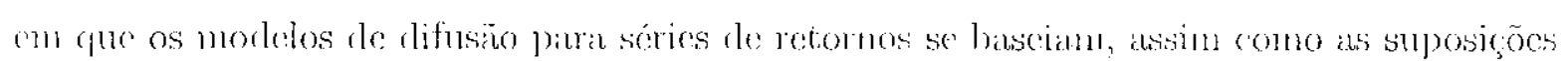

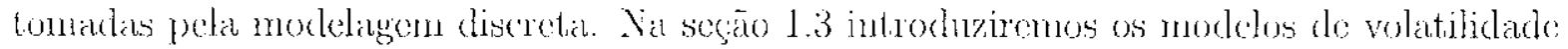

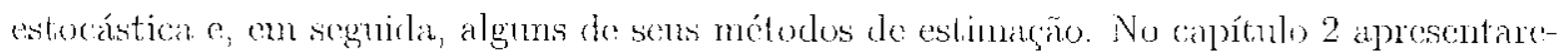
mos os modelos contínuos tratados neste traballo, algmmas de suas proprieflarles e tannbém

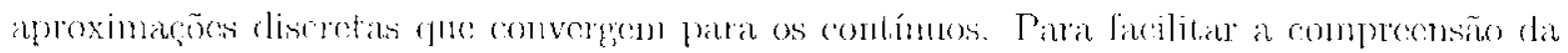
metorkologia aplicada aos molelos abordados neste traballo, traballaremos prineiramente?

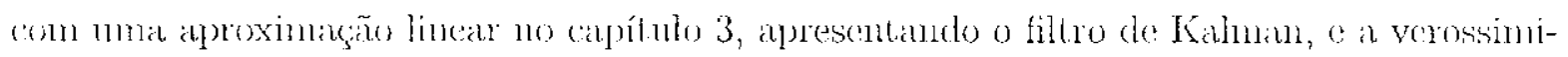

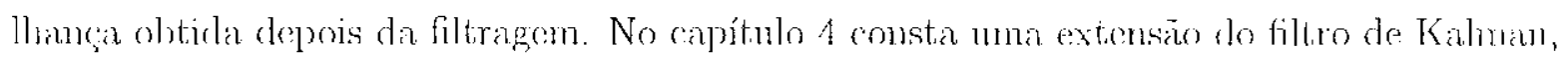




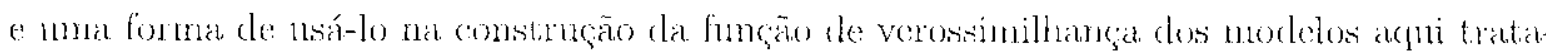
clos. Finalunente 110 (apjétulo 5 apresentaremos os resultados ampíricos relerentes a terceiro capútulo. No apêndice A consta a descriça dos métodos munéricos ntilizanlos neste traballio

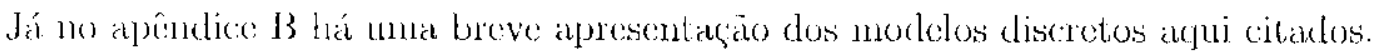

\subsection{Terminologia em Finanças}

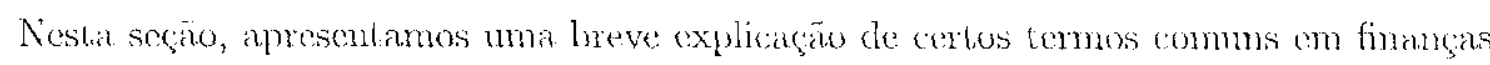
que sẫo usarles neste traballıo. Para mais delalles sugerinos (pex. Hull, 1996).

\subsubsection{Retorno}

Seja $S_{t}$ o preco de uma age no instante $l$, a retorno da ação no intervalo $(t-1,1), \Delta S_{t}$, é

$$
\Delta, S_{i}=S_{i} \cdots S_{i} \quad 1
$$

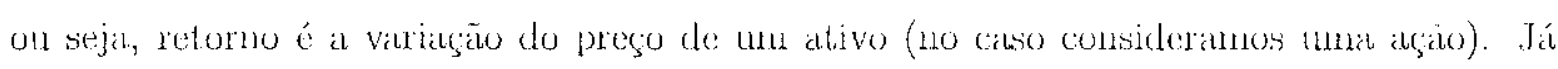

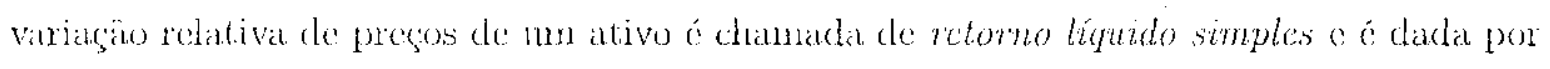

$$
R_{t}=\frac{S_{t} \ldots S_{t \cdots 1}}{S_{t}}=\frac{S_{t}}{S_{t-1}}-1 .
$$

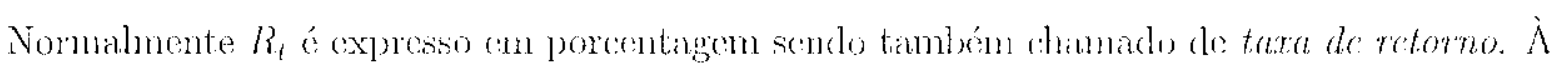
fracão $S_{1} / S_{1}$, diamos o none de retorno bruto simples.

É muito comum enfocarmos o retorno composto continumente on simplesmente logretorno de inna arian definido por

$$
I_{t}=\log \frac{S_{1}}{S_{t}}-\log S_{t}-\log S_{t-1},
$$

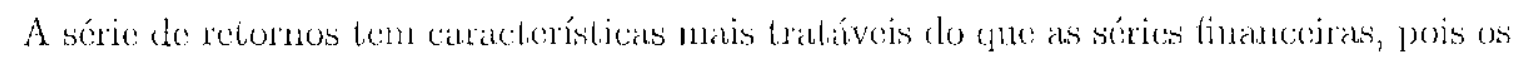

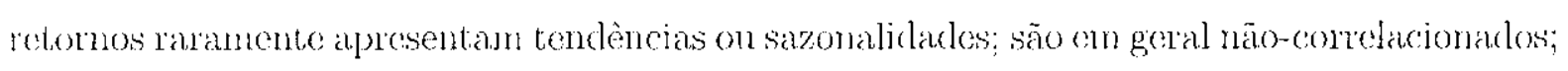

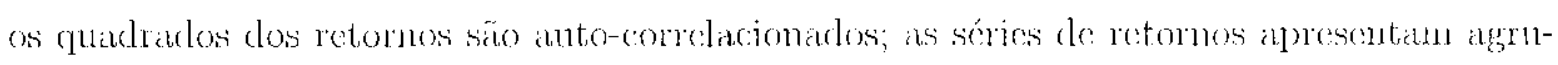

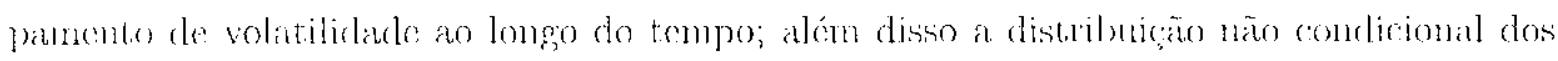

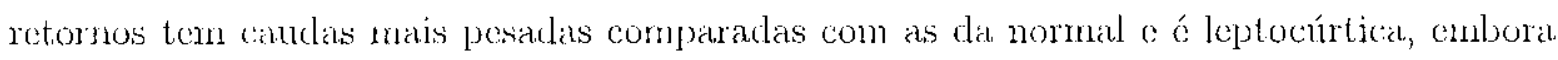
quase sinnétrica. Algumas dessas consideragões polen ser observadas comparando os gráficos

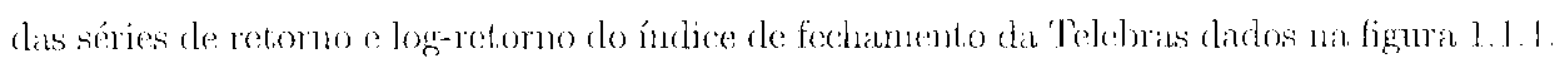




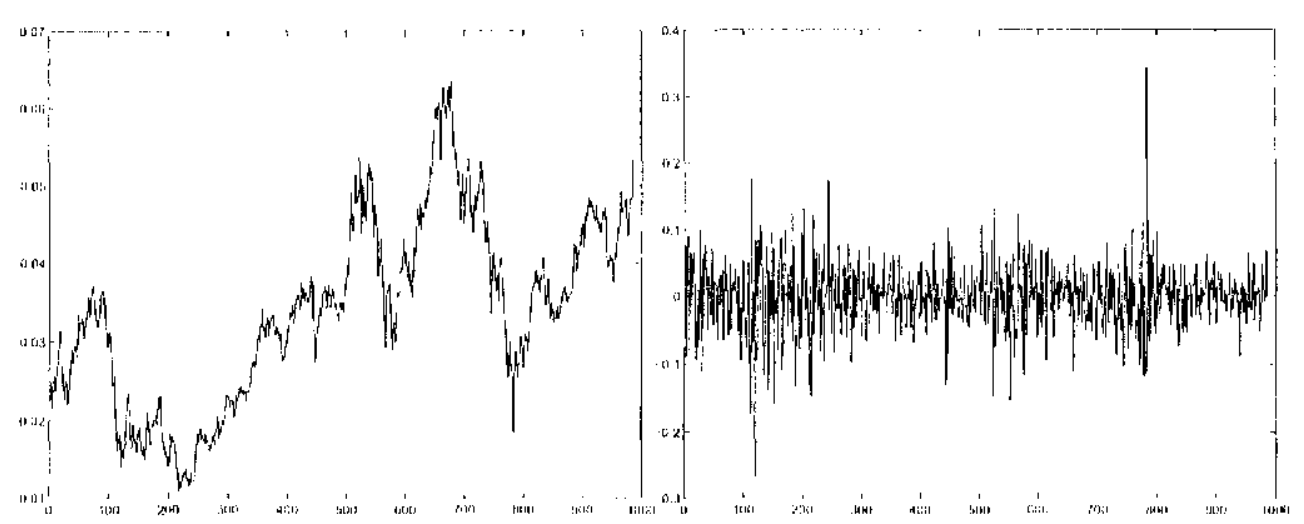

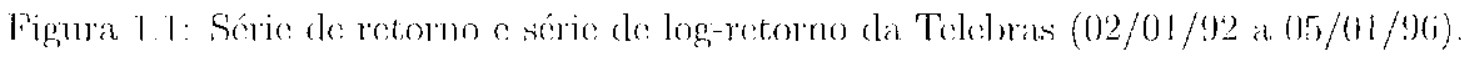

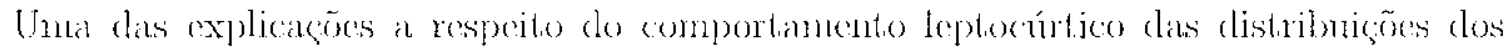

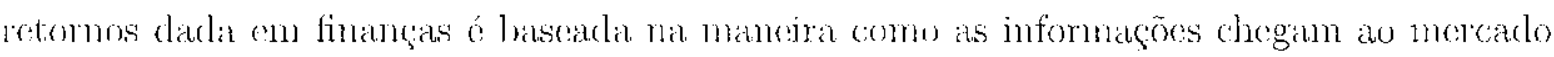

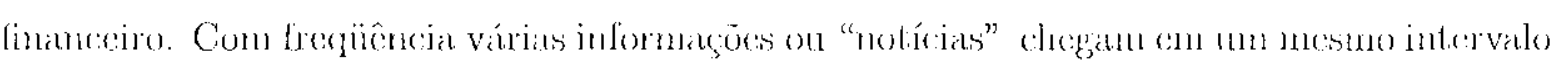

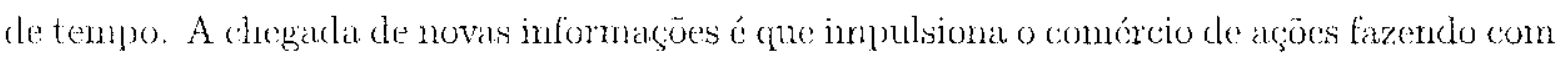

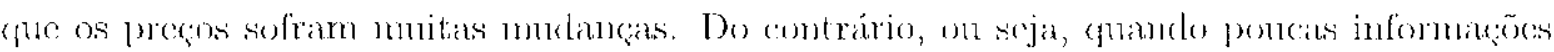

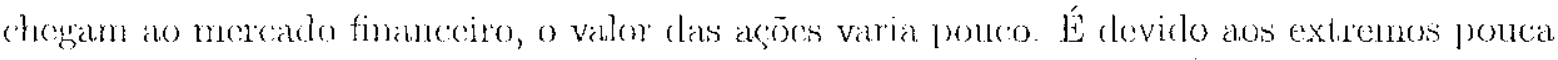

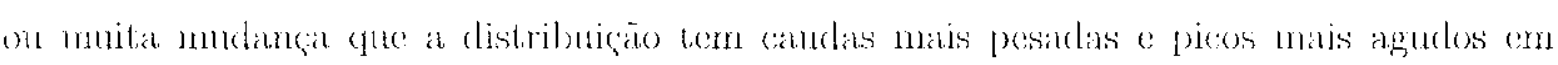
relaça aos da distribniçĩo nomal.

\subsubsection{Opção}

Suponhar que temhamos un título, chamado opgrio, no tempo $1: 20$ que nos dá o direito

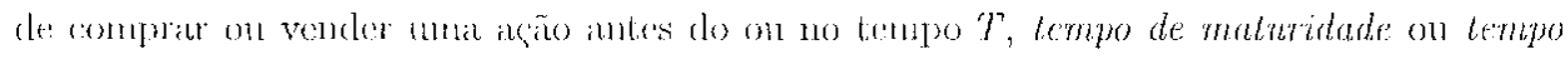

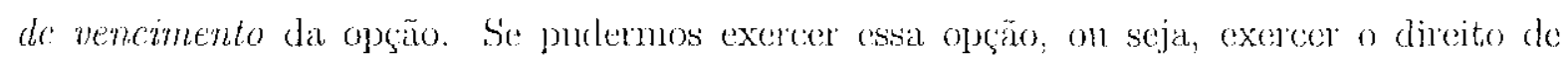

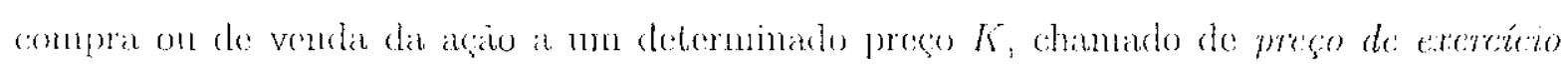

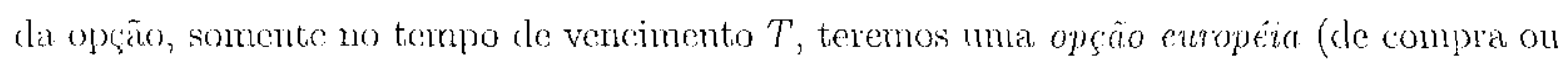

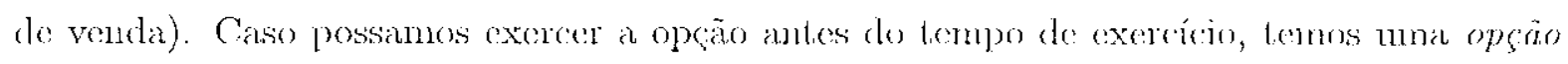

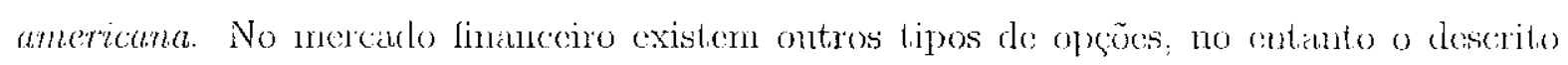

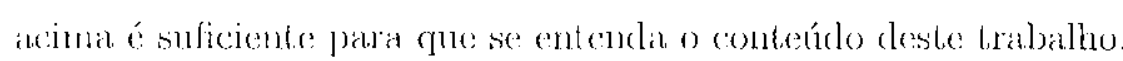

() titular do ma opgà não é obrigado a exercê-la. Pur exemplo, se no tempor T o preco

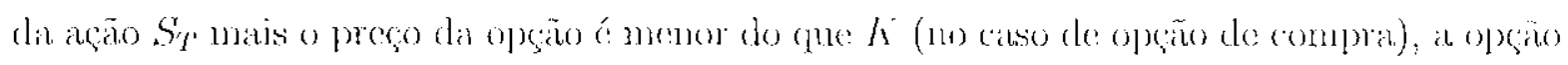

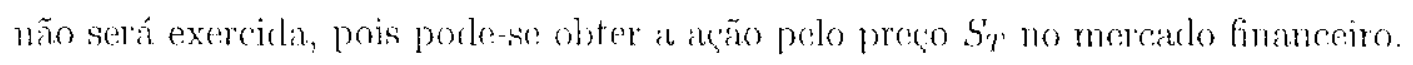




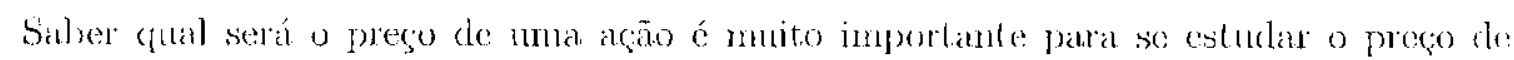

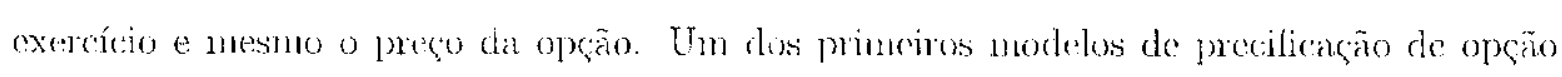
surgiu no traballio de Blark \& Scholes (1973) e se restringe a modelar o valor das opgöon te

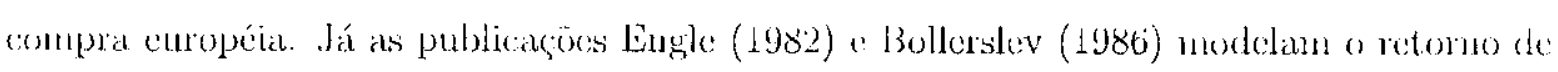

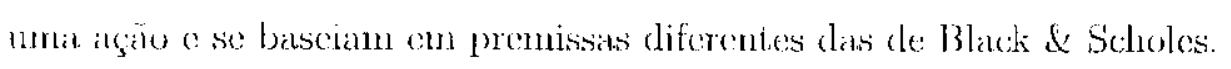

\subsubsection{Volatilidade em Mercados Financeiros}

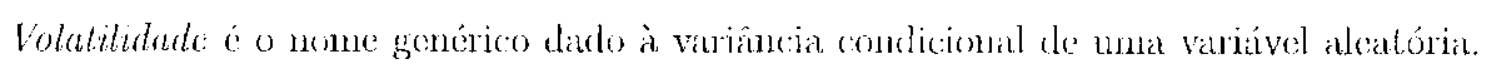

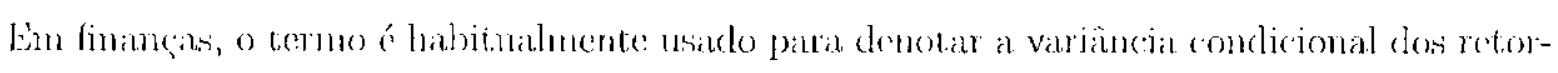

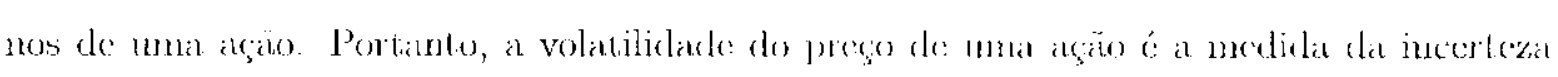

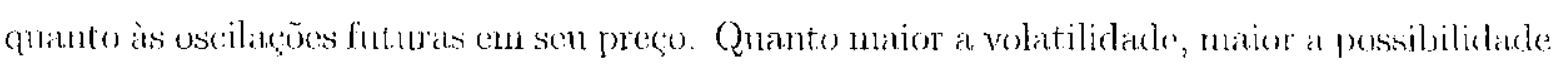

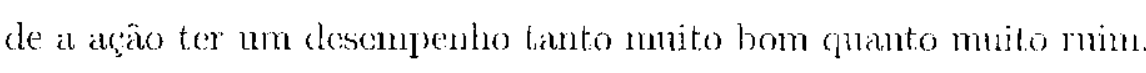

So o comportamento dos retornos fosse lomocedástico a normalunente distribuícto, mma estimativa para a volatilitale seria

$$
\sigma_{1}^{2}=\frac{\sum_{j=1}^{N-1} \Delta S_{j}^{2}}{N}, \quad \ell \because N, N+1, \ldots
$$

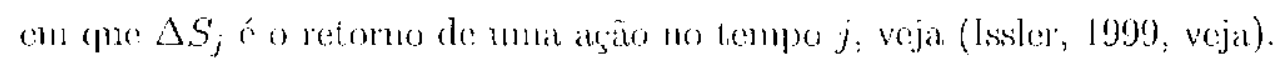

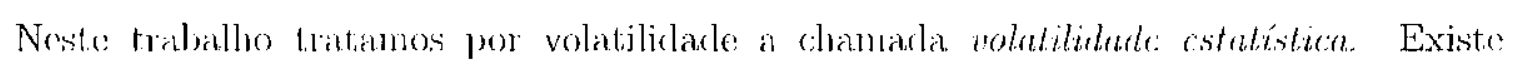

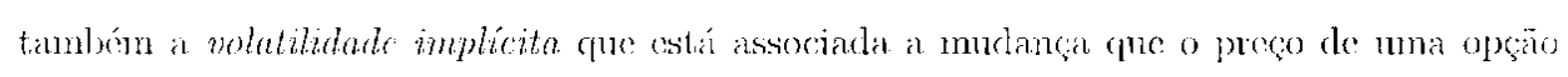

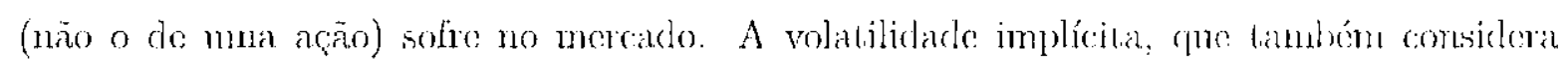

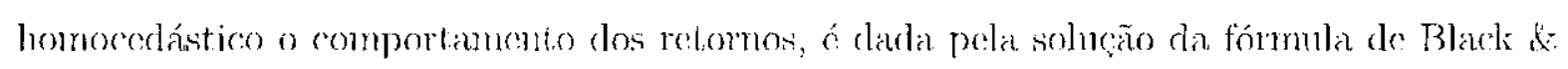

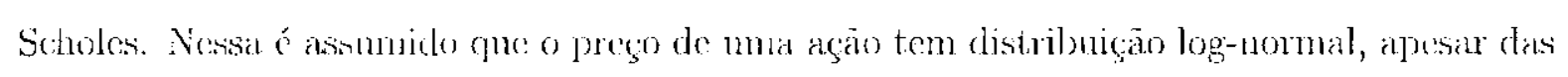

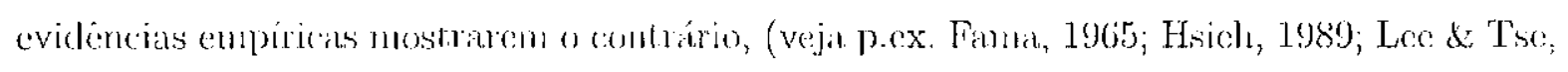

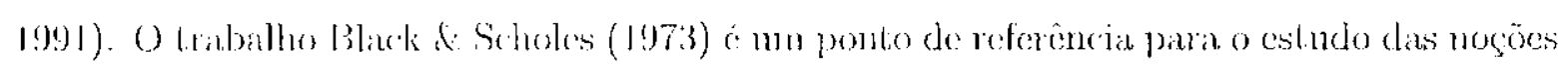

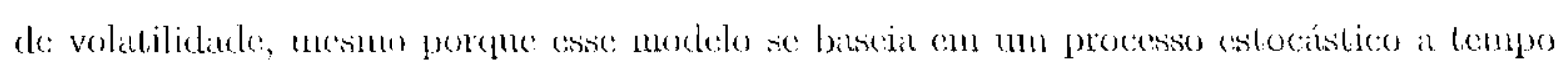

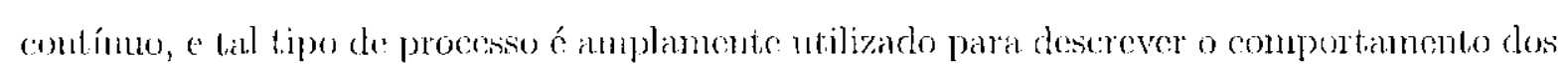
precsos de aroosis.

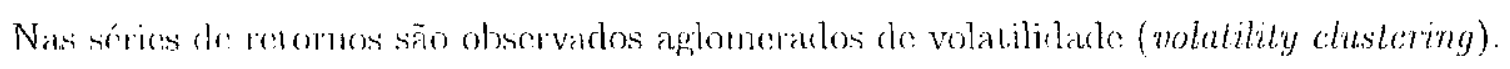

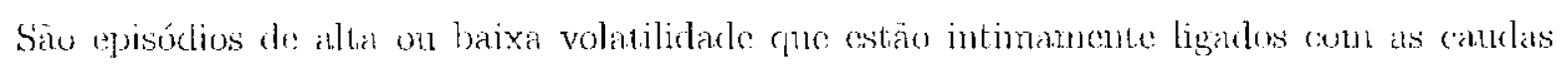
densas da distribuiçäo dos relonuos. A nomblagenn dessas séries é essencialmente construída

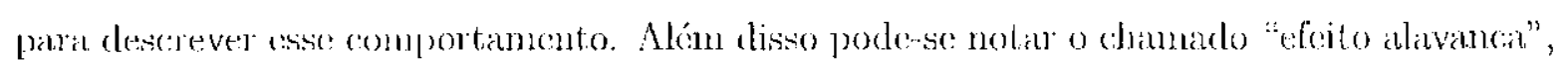


01 seja, a resposta da volitilidate é mais rápicla a retomos negalivos do que a retomon positivos.

\subsection{Dinâmica do Preço do Ativo}

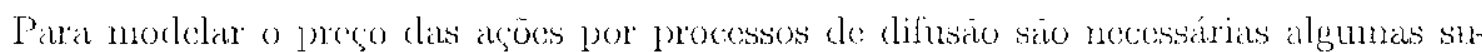
posiçoes iniciais. Vanos onsiderar um ativo financeiro, por exemplo uma aşo, com cotaço diária denotada por $S_{t}$. Seja $\Omega_{t}$ o conjunto de informacoúes obtidas até o instante l e consi-

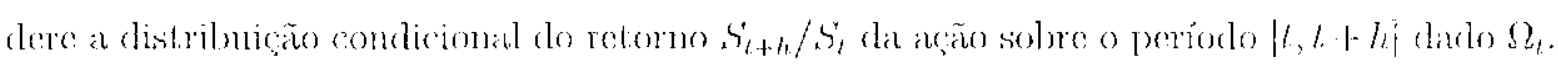
Varnus assumir que os retornos do alivo tem esperanga condicional finila, on seja,

$$
E\left(S_{i+h} / S_{t} \mid \Omega_{t}\right)=S_{t}^{-1} E\left(S_{i+h} \mid \Omega_{t}\right)<+\infty
$$

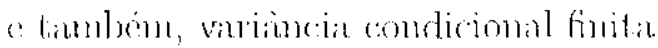

$$
\operatorname{Var}\left(S_{t+h} / S_{l} \mid \Omega_{t}\right)=S_{t}^{-2} \operatorname{Var}\left(S_{t+h} \mid \Omega_{l}\right)<+\infty
$$

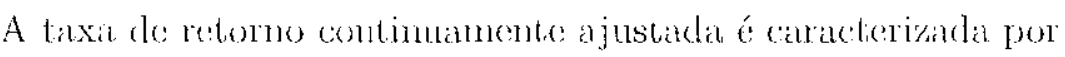

$$
h^{\prime} \log \operatorname{Es}\left(S_{t+1 / t}^{\prime} / S_{t} \mid \Omega_{t}\right)
$$

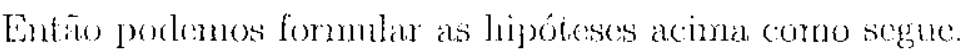

Hipótese 1.1. A taxa de retomo esperada continumente ajustada converge quase certamente para valores finitos, $\mu_{S}\left(\Omega_{i}\right)$, quando h tende a zoro pela direita. Temos então que

$$
E\left(S_{t+h !} \mid \Omega_{t}\right)-S_{t} \sim h_{1} \mu_{s}\left(S_{t}\right) S_{t}
$$

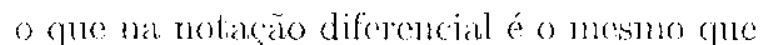

$$
\left.\frac{d}{d \tau} \mathrm{E}\left(S_{\tau} \mid \Omega_{t}\right)\right|_{\tau-t}=\mu_{s}\left(\Omega_{t}\right) S_{t}, \quad \text { quase certumente. }
$$

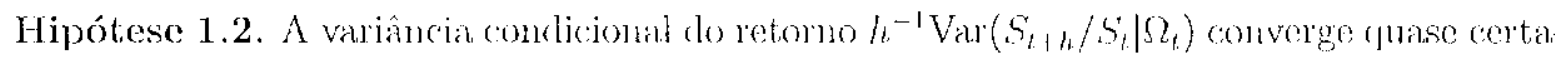

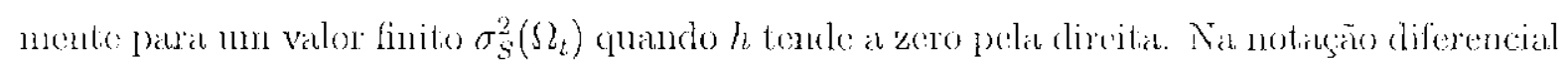
ticmos

$$
\left.\frac{d}{d \tau} \operatorname{Var}\left(S_{\tau} \mid \Omega_{l}\right)\right|_{\tau=l}-\sigma_{S}^{2}\left(\Omega_{i}\right) S_{l,}^{2} \text { quase certannente. }
$$


As hipóteses 1.1 e 1.2 nos levanı a representar a dinâmica do preço do ativo da seguinte forma

$$
d S_{t}=\mu_{S}\left(\Omega_{t}\right) S_{t} d t+\sigma_{S}\left(\Omega_{t}\right) S_{t} d W_{t}
$$

cm que $W_{t}$ é um movimento browniano. Logo, temos definido o chamado processo de volatilidade instantînea $\sigma_{s}\left(\Omega_{i}\right)$ que pode scr escrito, seguindo a notação arima, na forma

$$
\sigma_{S}\left(\Omega_{t}\right)=\left(\lim _{h \downarrow 0} h^{-1} \operatorname{Var}\left(S_{t+h} / S_{t} \mid \Omega_{t}\right)\right)^{1 / 2}
$$

O uso do movimento browniamo na precificaçã̃o de ação se justifica devido aos retonnos dos ativos não serem totalmente previsíveis. Isto se dá pela eficiente e quase que instantânea cleggada de iniornaçöes ao mercado financeiro provenicutes, al ualnente, do mundo inteiro. A demanda de informações que chegam ao mercado justifica também a suposição de que os retornos em períodos regulares de tempo, $[t+k, t+k+1], k=0,2, \ldots h-1$, são independentes c. identicamente distribuídos.

Vale notar que a fórmula de Black \& Scholes foi baseada num processo com $\mu_{S}\left(\Omega_{t}\right)=\mu_{S}$ e $\sigma_{S}\left(\Omega_{t}\right)=\sigma_{S}$ constantes para todo $t$, ou seja, bascada na idéía de que o preço da ação segue um movimerito browniano geométrico.

Agora consideremos que a dinâmica do preço do alivo é governada por varriávcis de csistado não ubservadas, como feito, de forma simplificada, no modelo de Full \& White (1987). Assim, assuninnos que unn processo de difusão $U_{t}$ descreve a variável de estado como

$$
\left\{\begin{array}{l}
d S_{t}=\mu_{\iota} S_{l} d t+\sigma_{t} S_{t} d W_{t} \\
d U_{t}=\gamma_{t} d t+\delta_{t} d W_{\iota}^{U} \\
\operatorname{Cov}\left(d W_{t}, d W_{t}^{U}\right)=\rho_{t} d l
\end{array}\right.
$$

em que $\mu_{t}, \sigma_{t}, \gamma_{t}$ e $\rho_{t}$ são como na hipótese a seguir.

Hipótese 1.3. $\mu_{l}, \sigma_{l}, \gamma_{t}$ e $\rho_{t}$ são $I_{t}^{U}=\left[U_{\tau}, \tau \leq t\right]$ adaptados.

A hipótese 1.3 nos clá que o processo $U$ idcntifica a dinâmica do preço do aljvo ś. E implica que, dada uma trajetória das variáveis de estado $\left(U_{\tau}\right)_{0 \leq \tau} \leq T$, os retomos consecutivos $S_{l_{k+1}} / S_{t_{k}:} 0 \leq t_{1}<t_{2}<\ldots<t_{k} \leq T$ são estocasticamente independentes e com distribuição log-normal. 


\subsubsection{Modelos Discretos}

Nesta segũo trabaremos de modelar a dinânica de um ativo por mu prosesso discreto no tempo análogo no processo descrito em (1.13). Isto também justifica a utilização da aproximax̧ão de Euler neste trabalho. Para maiores detalhes veja capítulo 2.

Unu unodelo discreto baseado no modelo (1.13) ó dado por

$$
\log \left(S_{i+1}^{i} / S_{i}\right)=\mu\left(U_{l}\right)+\sigma\left(U_{l}\right) \varepsilon_{i+1}
$$

Cono feito na modelagem contímua, devemos impor algumas hipótesces plantriveis capazes de assegurar a descrição das características das séries financeiras.

Hipótese 1.4. O processo $\varepsilon_{t}$ é independente e identicamente distribuído (i.i.d.) e também inckpendente da variável de estando do processo.

Considerando esta lipótese, podemos interpretar estatisticannente as funçōes $\mu\left(U_{t}\right)$ e $\sigma\left(U_{t}\right)$ como coeficiente de tendência e coeficiente de volatilirlade, respectivanente. Do fato,

$$
\begin{aligned}
\mathrm{E}\left[\log \left(S_{t+1} / S_{t}\right) \mid \Omega_{i}\right] & =\mathrm{E}\left[\mathrm{E}\left[\log \left(S_{t+1} / S_{t}\right) \mid\left(U_{\tau}, \varepsilon_{r}, \tau \leq t\right)\right] \mid \Omega_{t}\right] \\
& =\mathrm{E}\left[\mu\left(U_{t}\right) \mid \Omega_{\imath}\right],
\end{aligned}
$$

já que $\left.\mathrm{E}\left[\varepsilon_{l-1}\right\}\left(U_{\tau}, \varepsilon_{\tau}, \tau \leq l\right)\right]-E\left[\varepsilon_{l+1} \mid \varepsilon_{l}, \tau \leq l\right]=0$ devido a não influência de $U_{l}$ chn $\varepsilon_{1}$ de acordo com a hipótese acima. Da mesma forma mostramos que

$$
\begin{aligned}
\operatorname{Var}\left[\log \left(S_{t+1} / S_{t}\right)-\mu\left(U_{t}\right) \mid \Omega_{t}\right] & =\mathrm{E}\left[\left[\log \left(S_{t+1} / S_{t}\right)-\mu\left(U_{t}\right) \mid \Omega_{t}\right]^{2}\right]-[\underbrace{\mathrm{E}\left[\log \left(S_{t+1} / S_{t}\right)-\mu\left(U_{t}\right) \mid \Omega_{t}\right]}_{-0}]^{2} \\
& =\mathrm{E}\left[\left[\log \left(S_{t+1} / S_{t}\right)-\mu\left(U_{t}\right) \mid \Omega_{t}\right]^{2}\right]=\mathrm{E}\left[\left[\sigma\left(U_{t}\right) \varepsilon_{t+1}\right]^{2}\right] \\
& =\mathrm{E}\left[\sigma^{2}\left(U_{t}\right) \mid \Omega_{t}\right] \mathrm{E}\left[\varepsilon_{t+1}^{2} \mid \Omega_{t}\right]=\mathrm{E}\left[\sigma^{2}\left(U_{t}\right) \mid \Omega_{t}\right]
\end{aligned}
$$

enn que a penúltinza pabssalgem se dá pola hipótese acinal.

Hipótese 1.5. Seja $\Omega_{t}^{R} \equiv \sigma\left[S_{\tau} / S_{\tau-1}: \tau=0,1, \ldots, t-1, t\right]$ a $\sigma$-álgcbra gerada pelo conjunto de retomos passados. Suponhamos, então, $\mu\left(U_{t}\right)$ seja uma função $\Omega_{\ell}^{R}$ - mensumável. 
Inchindo essa última hipótese nas equações (1.15) e (1.16) concluímos que

$$
E\left[\log \left(S_{t+1} / S_{t}\right) \mid \Omega_{t}^{R}\right]=\mu\left(U_{l}\right)
$$

$$
\operatorname{Var}\left[\log \left(S_{i+1} / S_{t}\right)-\mu\left(U_{t}\right) \mid \Omega_{i}^{R}\right]=\mathrm{E}\left[\sigma^{2}\left(U_{t}\right) \mid \Omega_{l}^{R}\right]
$$

O trabalho Glıysels, Harvey \& Renault (1995) aborda a questão dia dinârnica dos preços de ativos, destacando o caso de o ativo ser ma opção.

\subsection{Modelos de Volatilidade Estocástica}

Os modelos de volatilidade estocástica (MVE) são bastante usados pela sua semelhança com os modelos habitualmente usados na teoria financeira. Comparados com os modelos do tipo ARCH, os MVE são capazes de descrever de forma mais adequada as propriedades observadas nas séries diárias de ativos financeiros, (veja p.ex. Carnero, Peña \& Ruiz, 2004). Além disso, na classe ARCTI é suposto que a variancia condicional depende dos retornos passakdos. Já no nodelo proposto inicialnnente por Taylor (1982), a volatilidade é descrita através de sens valores passados e independe dos retornos pasisados.

Consideremos agora um processo descrito por

$$
H_{t+1}=\mu_{\mathrm{t}}+\sigma_{t} \varepsilon_{t+1}
$$

em que $\mu_{t}$ é uma furıção mensurável pelo conjunto de observaçoes $H_{\tau} \in \Omega_{\tau}^{R}, \tau \leq t$. Pela equação (1.18) temos que

$$
\operatorname{Var}\left[H_{l+1} \mid \Omega_{l}^{R}\right]=\mathrm{E}\left[\sigma_{l}^{2} \mid \Omega_{l}^{l !}\right]
$$

sugerindo que

1. os aglomerados de volatilidade podem ser capturados por um processo com esperança condicional descrita por urn modelo auto-regressivo; e

2. as caudas clensas podem ser obtidas através cle erros $\varepsilon_{t}$ com distribuição de caudas pesadas ou por características estocásticas da $\mathrm{F}\left[\sigma_{t}^{2} \mid \Omega_{t}^{R^{2}}\right]$. 
Gcralmente um processo que detém as características dadas em (1) o (2) c um processo auto-regressivo de ordem $1, \operatorname{AR}(1)$, para alguma finnção não-linear de $\sigma_{l}$. Em suma, assumese que a volatilidade segue um processo de Markov de ordem um, não necessariannente linear en $\sigma_{t}$. Foi isso que motivou o trabalho de Andersen (1994) a introduzir a classe de modelos anto-regressivo de volitilidacle estocástica (MARVE).

Essa classe engloba vários modelos já conhecidos na literatura, como é o caso do proposto por Taylor (1982), un modelo anto-regressivo de variância alcatória descrito por

$$
\begin{aligned}
y_{l} & =\sigma_{l} \varepsilon_{l} \\
\log \sigma_{l} & =\alpha+\phi \log \sigma_{l-1}+\eta_{l},
\end{aligned}
$$

sendo $\eta_{t}$ um rúdo branco

A forma canônica de Kinn, Kim, Shcphard \& Chib (1998), também faz parte da classe MARVE e é descrila por

$$
\begin{gathered}
y_{l}=\sigma^{*} e^{\nu_{t} / 2} E_{l} \\
\nu_{t+1}-\alpha=\beta\left(\nu_{l}-\alpha\right)+\sigma_{\eta} \eta_{t},
\end{gathered}
$$

en que $\varepsilon_{t} \mathrm{e} \eta_{t}$ săo normalmente distribuídos com módia zero e variância unitária

O MVE mais popular na litcratura dentro desse contexto é também um MARVE. Eé clado por

$$
\begin{aligned}
& y_{t}=\mu_{t}+\sigma^{*} e^{\nu_{t} / 2} \varepsilon_{t} \\
& \nu_{t}=\phi \nu_{t-1}+\sigma_{\eta \eta} \eta_{l},
\end{aligned}
$$

enn que $\nu_{t}=\ln \left(\sigma_{t}^{2} / \sigma^{* 2}\right)$. Mais detalhes a respeito deste tipo de modelo serão dados na seção 2.3.1.

As propriedaules dos MARVE tôm sido cstudadas em vários trabalhos (veja p.cx. Taylor, 1994; Barndorff-Nielsen \& Shephard, 2001). Há também traballos que, ao invés de tomarem crros $\varepsilon_{t}$ com distribuição Normal, assumem erros com distribuiçōes de caudas pesadas, (veja p.ex. Harvey, Ruiz \& Shcphard, 1994; Sandmann \& Koopman, 1998; Watanabe \& Asai, 2001). No entanto, vale ressaltar que os trabalhos Andersen, Bollerslev, Dicbold \& Labys (2001) e Andersen, Bollerslev, Diebold \& Lábys (2003) mostran que a log-volatilidade pode ser aproximarla por uma distribuição normal, ou scja, considerar erros ganssianos é adecurado. 
A inuportância dos MARVE barmbém se dá por poder incorporar o efeito alavanca (conentade na segäo 1.1.3). Para isso, o trabahlo Ilarvey \&. Shephard (1996) propóc que os erros $\varepsilon_{1}$ e nl.., do MARVE sejan negativanente conelacionalos. Para o mesmo tim, o mabalho de Jacquier, Polson \& Rossi (2002) sugere que os elros $\varepsilon_{t}$ e th sejan correlacionados.

\subsection{Métodos de Estimação}

Ao contrário do que ocorre con os modelos ARCH, a lingrio de verussinnilhanga dos MVE

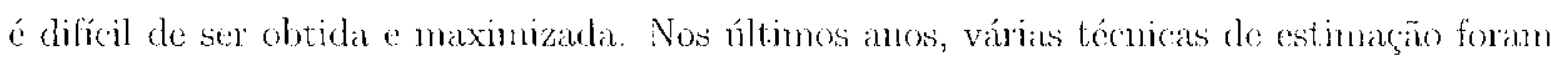
entudatas a fim de suprir estas dificuldacles e permitir as aplicacões empíricas dos MVE.

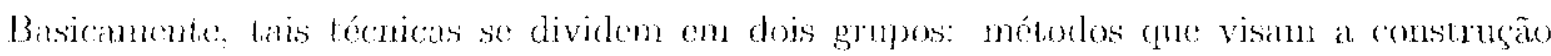
da verosimilhanga exata e unétodos que buscan aproxinita on simplesmente a evitam. Thabalhos cono Kim et al. (1998) e Sandmann \& lioopman (1998) se encaixam no primeiro grupo. O método da quase-verossimilhanģa nsado por Harvey et at. (1991), assim como o método dos monentos originahnente proposto por Taylor (1986) săto cxtemplos de iécnicals do segmudo grupo.

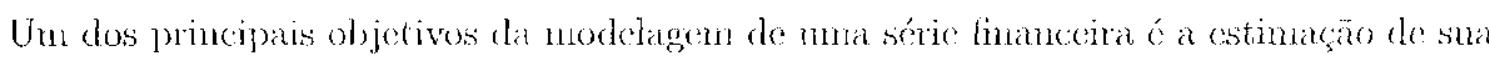

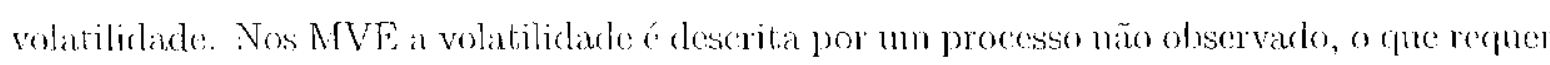

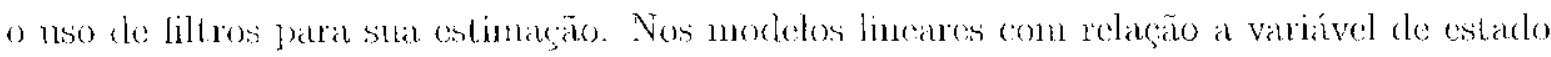

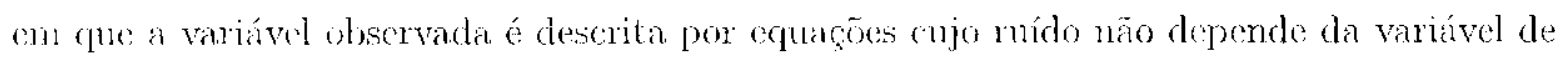
cstarlo, o nso do liltro de Káman (FK) cumpre o objetivo em questão. Os MVE não possuen

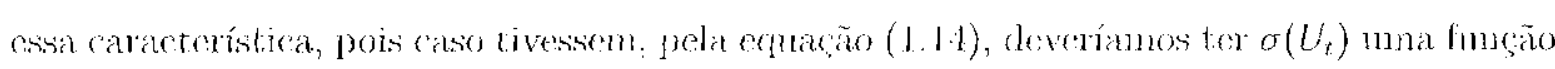

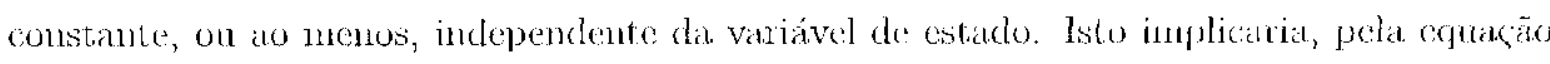

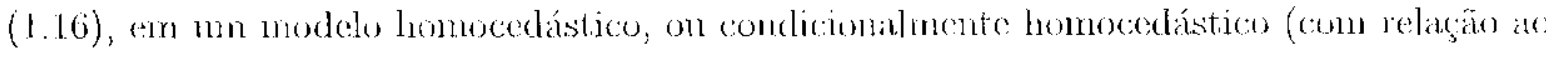

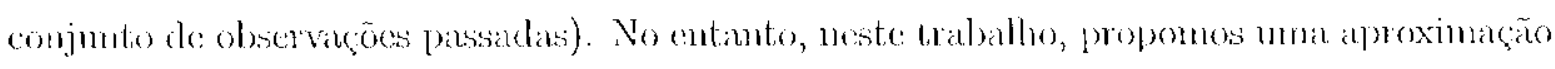

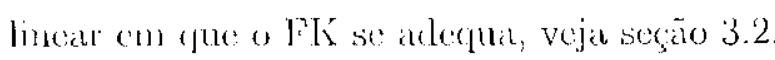

Quando näo temos modelos lincares nas ainda que teuhan rujdo indejendente da variável

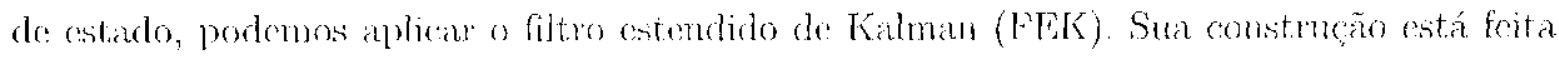
no capítulo 4. No entanto, nos modelos tratados neste traballos, alén da linearidarle näo

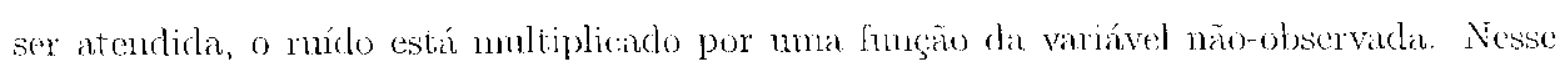

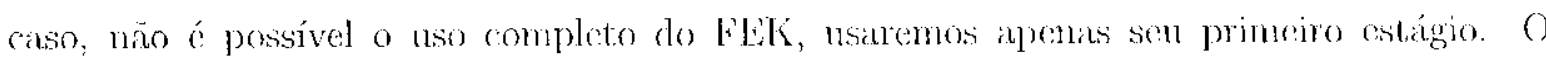




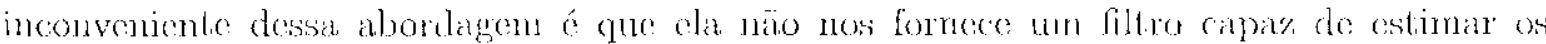

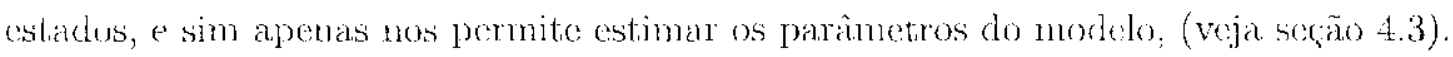

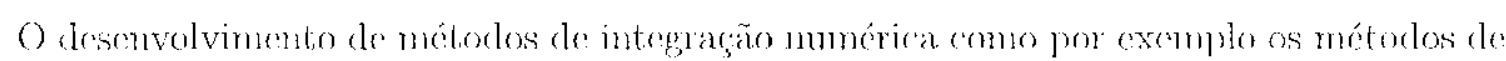

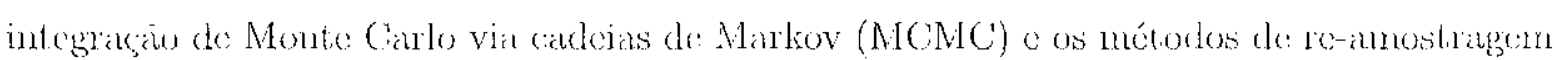

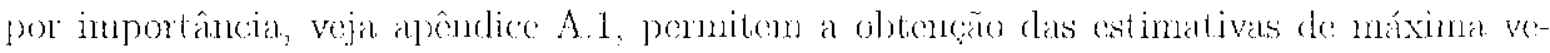

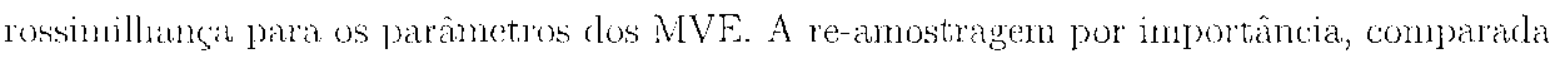

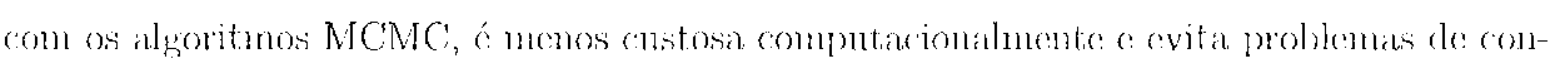
vergência. Além disso, suas estimativas podem ficar mais exatias anmentando o numero

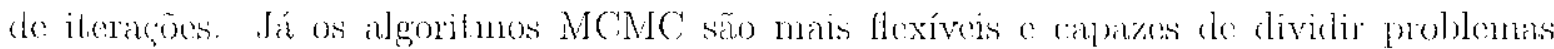
de dimenoos altas em casos de monor dimensão. O trabalho de Sandmann \& Koopman

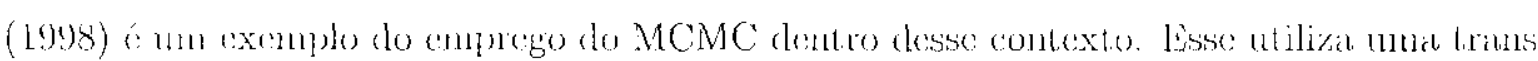

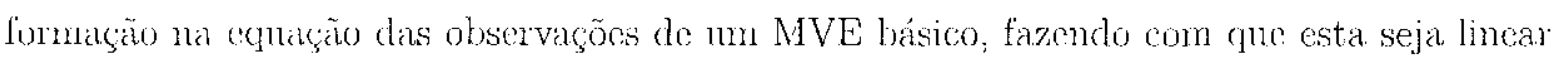

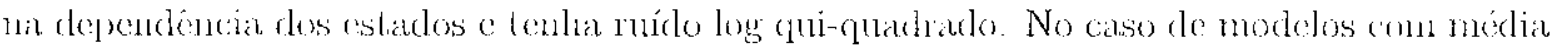

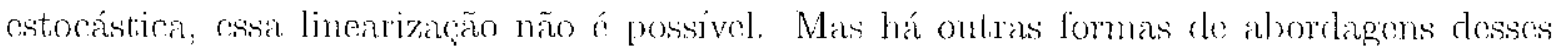

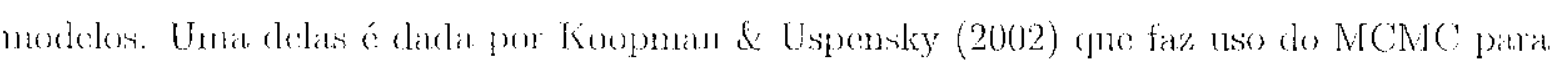
se obteren as estimativas de máxina verossimilhanga.

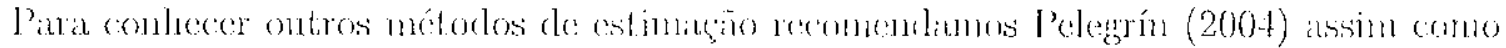
os tirabahos nele citados e Ghysels el al. (1995). 


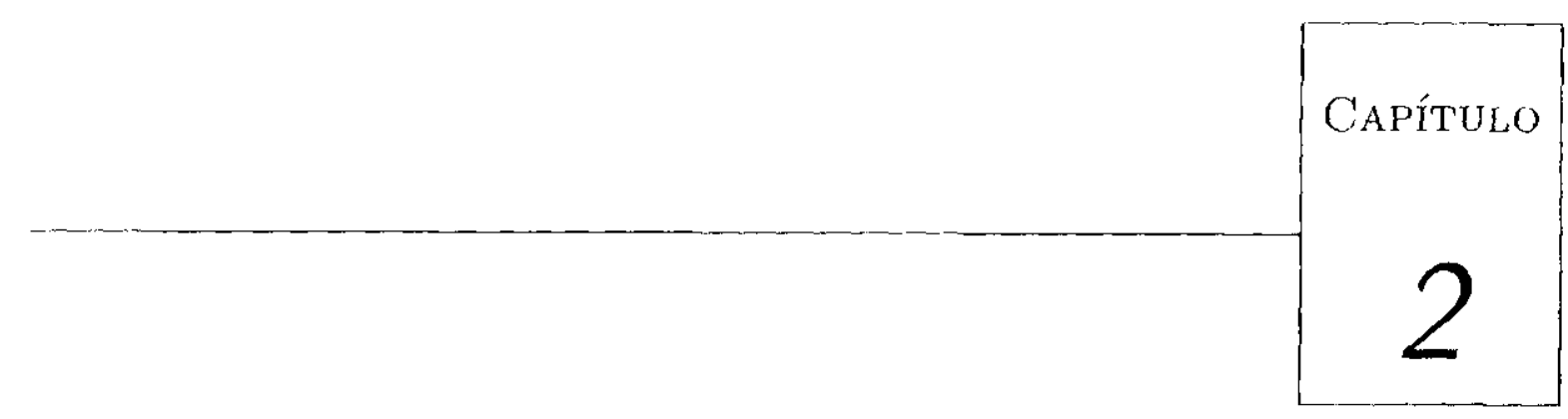

Modelos I e II

Apresentaremos aqui os dois modelos enfocados neste traballio, juntanente com suas aproximaçöss discretats que seguem primeiranente, pois algunnas de snas propricdades dependem delas. Antes disso, introduzimos alguns conceitos básicos para o entendimento do capítulo, baseados em Morettin \& Toloi (2004), Cinlar (1975) c Økscndal (1998)

\subsection{Preliminares}

Definição 2.1 (Processo Estocástico). Seja $T$ um conjunto arbitrário. Um processo estocástico ó unra fanńlia $Z=\{Z(t), t \in T\}$, tal que, para cada $t \in T, Z(t)$ é uma variável aleatória.

Informalmente podemos dizer que um proccsso estocástico é o conjunto de todas as possíveis trajetórias de um certo processo. Da definição formal conchúmos que processo estocástico é uma família de variávois aleatórias. 
Definição 2.2 ( $\sigma$-álgebra). Scja $\Omega$ um conjunto qualç̣ter. Então a $\sigma$-álgebra $\mathcal{F}$ com relação a $\Omega$ é una farnília $F$ de subconjumtos de $\Omega$ com as scguintes propricelades

(i) $\emptyset \in \mathcal{F}$

(ii) $F \in \mathcal{F} \Rightarrow F^{C} \in \mathcal{F}$, en que $F^{C}$ denota o conjunto complementar de $F$ em $\Omega$;

(iii) $A_{1}, A_{2}, \ldots \in \mathcal{F} \Rightarrow A \doteq \bigcup_{i=1}^{\infty} A_{i} \in \mathcal{F}$.

Definição 2.3 (Processo Mensurável). Considere o espaç̧o de probabilidades $(\Omega, \mathcal{F}, P)$. Dizenos cue $X: \Omega \rightarrow \mathbb{R}$ é um processo mensurável com relação a $\mathcal{F}$, ou simplesmente $\mathcal{F}$-monsurável, se e somente se $\{\omega: X(\omega)<a\} \in \mathcal{F}$, para todo $a \in \mathbb{R}$.

Definiçāo 2.4 (Processo Adaptado). Seja $\mathcal{M}_{t}$ uma família crescente de $\sigma$-álgebras de: subconjuntos de $\Omega$. Um processo $B_{t}(\omega) \in \mathbb{R}$, para $t \geq 0$ e $\omega \in \Omega$, é un processo adaptado

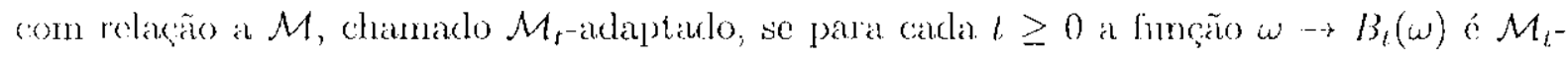
mensurável.

De maneira simples, podemos dizer que urn processo $B_{t}(\omega)$ e $\mathcal{M}_{t}$-adlaptado quando as informações "contidlas" na $\sigma$-álgebra referente são suficientes para "descrevê-lo".

Definição 2.5 (Processo de Markov). Um processo cstocástico $\left\{X_{n}, n \in \mathbb{N}\right\}$ ć 1 um processo de Markov com espaço de estado E enumerável e finito se

$$
\Gamma\left(X_{n+1}=j \mid X_{0}=i_{0}, X_{1}=i_{1}, \ldots, X_{n}=i_{n}\right)=P\left(X_{n+1}=j \mid X_{n}=i_{n}\right)
$$

para todo $n \in \mathbb{N}$ e $i_{0}, i_{1}, \ldots, i_{n} \in E$

De mancira informal, podemos dizer que ưn processo estocéstico é um processo de Markov se a probabiliclade de movimento para $j$ depende apenas do estágio imediatamente anterior, $i_{n}$, ou sejal, não importa a trajctória, e sim o "tamanho" do passo.

Definição 2.6 (Processo de Wiener). O processo de Wiener ou movimento brow-

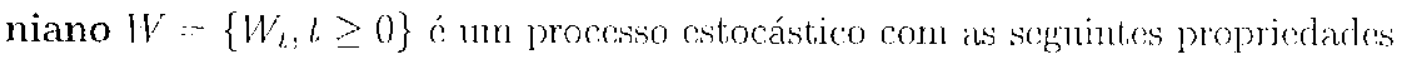

(i) $W_{0}=0$; 
(ii) os increnentos $W_{l}-W_{s}$ são estacionádios e independentes;

(iii) para todo $t>0, W_{t} \sim \mathcal{N}(0, t)$;

(iv) as trajetórias são contímuas quase certamente (q.c.).

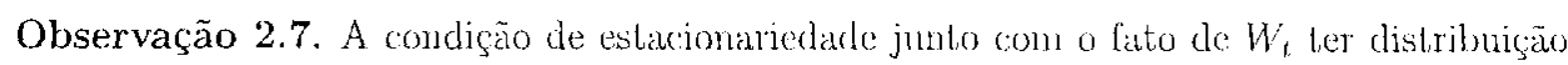
normal implicam que $W_{l}-W_{s}$, para $t>s$ tern distribuiçäo normal com módia zcro e variânncia $t-s$.

Definição 2.8 (Equação Diferencial Estocástica). Umà equação diferencial estocástica (EDE) dada por

$$
d X_{l}=a\left(X_{\iota}, t\right) d t+b\left(X_{t}, l\right) d W_{l} .
$$

cm que $a\left(X_{t}, l\right)$ e $b\left(X_{t}, l\right)$ são funções conhecidas, é apenas umba forma aloreviada de representar a equação integral estocástica

$$
X_{l}=X_{0}+\int_{0}^{t} a\left(X_{s}, s\right) d s+\int_{0}^{t} b\left(X_{s}, s\right) d W_{s} .
$$

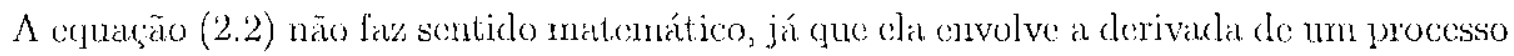
de Wiener que não tem derivada en quase todo ponto (exceto cono processo generalizado). Representando a EDE na forma (2.3) damos sentitido a cla, pois sua solução pode sor dada explicitanente. Sobre certas condiçoes impostas às equaçoos $a\left(X_{i}, l\right)$ e $b\left(X_{i}, l\right)$ é possível mostrar que a EDE tern uma única solução $X_{t}$, veja $\varnothing$ ksendal (1998). Tal solnção é unu processo estocástico o não uma soluçĩo determinústica.

Definição 2.9 (Aproximação de Euler). Seja $X_{t}, t_{0} \leq t \leq T$ um processo de Itố tal cono em (2.42) e com condição inicial $X_{t_{0}}=X_{0}$. Tomemos uma discretização $t_{0}<\ell_{1}<$ $\ldots<l_{n} \ldots<l_{N}=T$ do intervalo de tempo $[0, T]$. Seja $Y=Y(l)$ para $l_{0} \leq t \leq T$ ' un processo estocástico contínuo no tempo con condição inicial $Y_{0}=X_{0}$, e tal que

$$
Y_{n+1}=Y_{n}+a\left(t_{n}, Y_{n}\right)\left(t_{n+1}-t_{n}\right)+b\left(t_{n}, Y_{n}\right)\left(W_{t_{n-1}}-W_{n}\right),
$$

para $n=0,1, \ldots, N-1$, cur que $Y_{n}=Y\left(b_{n}\right)$. O processo $Y$ ó o que chamamos de aproximação de Euler, on scja, an aproximaçāo de Enler ć uma técricu básica de discretização de unı processo de Itô. 


\subsection{Modelo discreto por modelo de difusão}

A molelagenn contínua ó capaz de representar as premissas teúricas de precificaryou de ativos. No cntanto, a coleta dos dados somente pole ser feita em intervalos cliscretos de tempo. Temos então de $1 \mathrm{~m}$ lido um modelo teórico contíno e de ontro dados reais discretos. Uma possibilidarle de unir a teoria à prática é procurar um processo linite para um processo discreto, ou seja, mostrar gue a prática pode se aproximar da teoria. Nesta seção apresentaremos condiçöes gerais sob as quais uma seqüûncia de processos de Markov discretos no tempo converge fracamente para um processo de Itô.

Primeiramente vamos construir um processo contínno no tempo a partir de um processo de: Markov discreto no tempo. Dado $h>0$ anditrário, comsidere o processo de Markov discreto no tempo $X_{0}, X_{h}, X_{2 h}, \ldots, X_{h h}$ denotado por $\left\{X_{k h}\right\}$, on que $X_{k h}$ toma valores no $\mathbb{R}^{n}$ para todo $k$. Assmma que sejam conhecidas as probabilidades de transição de $\left\{X_{k h h}\right\}$ o a distribuçãa da variável inicial $X_{b}$, O) processo contímo no tempo $\left\{X_{t}^{(h)}\right\}$ é consimúrlo através do processo discreto $\left\{X_{h h}\right\}$ fazendo $X_{l}^{(h)}$ man funçäo step com saltos nos tempos $h, 2 h, \ldots$, o tal que $X_{i}^{(h)}=X_{k h}$ quase certamente para $k h \leq t<(k+1) h$. Sejanı

$$
\mu_{h}(x) \equiv h^{-1} \mathrm{E}\left[\left(X_{h+3}^{(h)}-X_{h}^{(h)}\right) \mid X_{k}^{(h)}=x\right]
$$

$$
\Sigma_{h l}(x) \equiv h^{-1} \operatorname{Cov}\left[\left(X_{h+1}^{(h)}-X_{k}^{(h)}\right) \mid X_{k}^{(h)}=x\right]
$$

Considexe também gue $F_{h}\left(X_{0}^{(h)}\right)$ denota a fumção distribuiçäo acmundada de $X_{0}^{(h)}$.

Teorema 2.10 (Stroock e Varadhan (1979)). Seja $\left\{X_{t}\right\}$ un processo governado pela seguinte equaçio integral cstocástica

$$
X_{t}=X_{0}+\int_{0}^{t} \mu\left(X_{s}\right) d s+\int_{0}^{t} \Sigma^{l / 2}\left(X_{s}\right) d W_{s}
$$

en que $W_{t}^{\prime}$ é um movinento browniano pactrão, $\mu(\cdot)$ ć uma função contínua do espaço daśs matrizes rodis $N \times N$. Supouha quwe a integral em (2.7) lenha solucão fraca ínica. So

1. $F h(\cdot) \cdots F(\cdot)$ quando $h \perp 0$ para todo ponto de contimuidade de $F(\cdot)$

2. $\mu_{h}(\cdot) \rightarrow \mu(\cdot)$ unilormencute para todo conjunto limitado kle $x$ quando $h \perp 0$ 


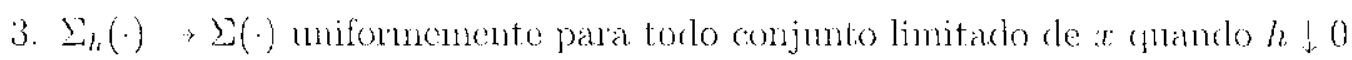

1. $\exists \delta>0$ tal ane $h^{-1} E\left[\left\|X_{h+1}^{(h)}-X_{k}^{(h)}\right\|^{2-\gamma)} \mid X_{k}^{(h)}=x\right] \quad, 0$ miformemente para lodo

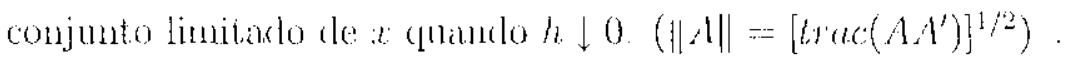

Então $\left\{\lambda_{t}^{(h)}\right\}$ converge fracanente para $\left\{X_{l}\right\}$ quando $h \downarrow 0$.

As hinóteses arina equivilem a dizer yue

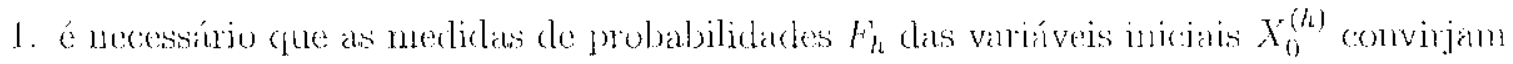
para una mexlicla limite F quando $h\rfloor 0$;

2. a tendencia do processo $\left\{X_{l}\right\}$, $\mu_{h}$, e a matriz de difusão, $\Sigma_{h}$, deven convergir mifor-

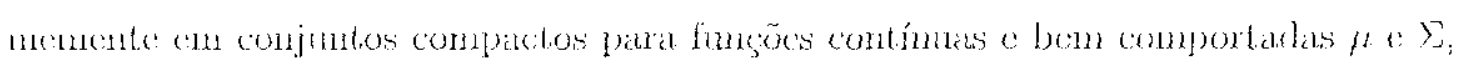
resiecelivanente:

3. As diferencicts do processo $X_{k}^{(h)}$ deven ter pelo menos un dos monentos absolutos de orden matur que dois, por mondade de tempo, convergincio para zero a mua tinxa njopriala yuamio h. 10 .

\subsubsection{Modelo I}

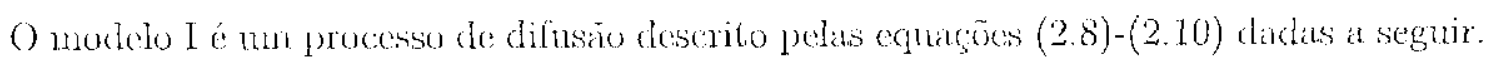

$$
\begin{aligned}
d H_{t} & =\left(\mu-\frac{\mathrm{e}^{\nu_{t}}}{2}\right) d t+\mathrm{e}^{\nu_{t} / 2} d \mathrm{~W} \\
d \nu_{t+h} & =\beta\left(\alpha-\nu_{t}\right) d l+\gamma d \mathrm{~W}_{t}, \text { con } \nu_{t}=\ln \sigma_{t}^{2},
\end{aligned}
$$

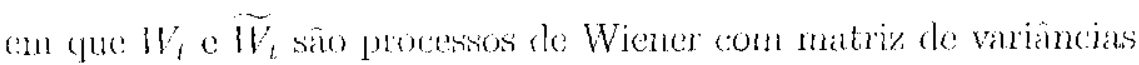

$$
\operatorname{Var}\left(\begin{array}{l}
W_{t} \\
W_{t}
\end{array}\right)=\left(\begin{array}{ll}
1 & \beta \\
\beta & 1
\end{array}\right) \text {. }
$$

Agora comidere o processo a tompo discreto dado por

$$
\begin{aligned}
& H_{t+h}=H_{t}+h\left(h-\frac{e^{\nu_{t}}}{2}\right)+h^{1 / 2} e^{\nu_{t} / 2} Z_{t+h} \\
& \nu_{t+h}=\nu_{t}+h \beta\left(\alpha-\nu_{t}\right)+h^{1 / 2} \gamma \ddot{Z}_{t+h}
\end{aligned}
$$




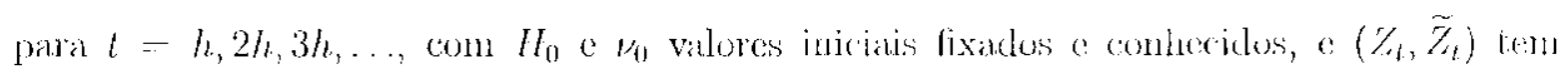
(listribuiçäo normal bivariada independente e identicanente distribúda (i.i.d.) com vetor de méclias $(0,0)$ o matrì de variuncia

$$
\operatorname{Var}\left(\begin{array}{c}
Z_{i} \\
\tilde{Z}_{i}
\end{array}\right) \cdots\left(\begin{array}{ll}
1 & \rho \\
\rho & 1
\end{array}\right) .
$$

Definanos os procesisos contímos no temupo $/ l_{\tau}^{(h)}$ e $\mu_{\tau}^{(t)}$ por

$$
H_{\tau}^{(h)}=H_{l} \quad \text { o } \quad \nu_{\tau}^{(h)}=-H_{t}, \quad \text { paral } \quad l \leq \tau<t-1 h .
$$

Seja $\mathcal{M}_{t}$ a $\sigma$-álgcha geralda jor $\left\{H_{\tau}, \nu_{\tau}, 0 \leq \tau \leq \ell\right\}$. Desta forma

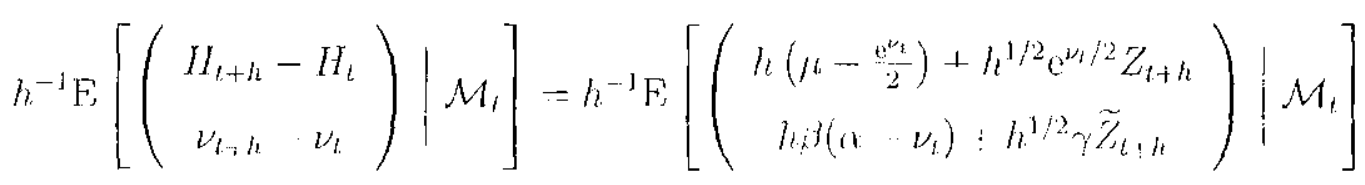

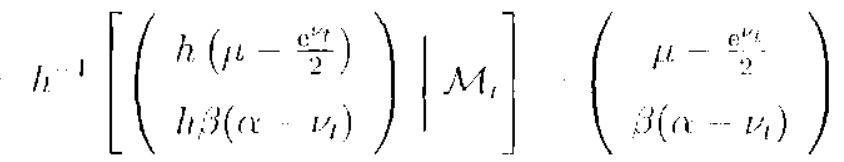

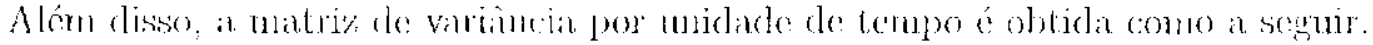

$$
\begin{aligned}
& h^{1} \operatorname{Var}\left[\left(\begin{array}{c}
H_{t+h}-H_{t} \\
\nu_{t+h}-\nu_{t}
\end{array}\right) \mid \mathcal{M}_{t}\right]=h^{-1} \operatorname{Var}\left[\left(\begin{array}{c}
h^{1 / 2} \mathrm{c}^{t^{2} / 2} Z_{l+h} \\
h^{1 / 2} \gamma \bar{Z}_{t+h}
\end{array}\right) \mid \mathcal{M}_{t}\right] \\
& \operatorname{Var}\left[\left(\begin{array}{cc}
\mathrm{c}^{\nu_{t} / 2} & 0 \\
0 & \gamma
\end{array}\right)\left(\begin{array}{c}
Z_{l} \\
\widetilde{Z}_{l}
\end{array}\right) \mid \mathcal{M}_{l}\right]=\left(\begin{array}{cc}
\mathrm{c}^{\nu_{t} / 2} & 0 \\
0 & \gamma
\end{array}\right) \operatorname{Var}\left(\begin{array}{c}
Z_{l} \\
\ddot{Z}_{t}
\end{array}\right)\left(\begin{array}{cc}
\mathrm{e}^{\nu^{\prime \prime} / 2} & 0 \\
0 & \gamma
\end{array}\right) \\
& =\left(\begin{array}{cc}
e^{\prime \prime t} & \gamma \rho \mathrm{c}^{\prime^{\prime \prime}} \\
\gamma \rho \mathrm{e}^{\nu_{t}} & \gamma^{2}
\end{array}\right) \text {. }
\end{aligned}
$$

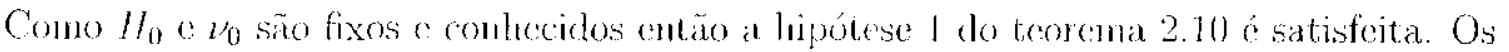

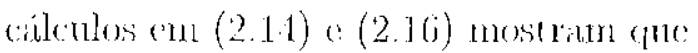

$$
\mu_{h}\left(\left(\mu_{l}, \nu_{l}\right)\right)=\left(\begin{array}{c}
\mu--\frac{e^{\prime \prime} t}{2} \\
\beta\left(\alpha-\nu_{l}\right)
\end{array}\right)
$$

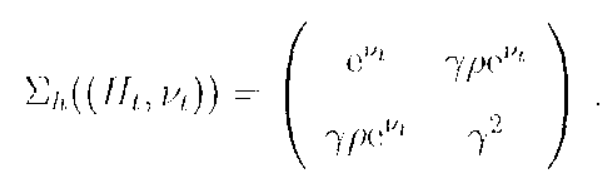


E isto nos dá que $\mu_{h}\left(\left(H_{t}, v_{t}\right)\right)$ e $\Sigma_{h}\left(\left(H_{t}, \nu_{l}\right)\right)$ não dependem de $h$, portanto convergem uniformemcnte en qualquer conjunto limitado de $\left(H_{l}, \nu_{t}\right)$ quando $h \downarrow 0$. Logo são satisfeitas as condiçōos 2 o 3 do tcorema 2.10. É fácil, embora trabalhoso mostrar que

$$
\begin{aligned}
\mathrm{E}\left[h^{-1}\left(H_{t+h}-I I_{l}\right)^{4} \mid \mathcal{M}_{t}\right]=h^{3}\left(\mu-\frac{\mathrm{e}^{\nu_{t}}}{2}\right)^{4} & +4 h^{2}\left(\mu-\frac{\mathrm{e}^{\nu_{t}}}{2}\right) \mathrm{e}^{\nu_{t}}+3 h \mathrm{e}^{2 \nu_{t}} \\
& +2 h^{2}\left(\mu-\frac{\mathrm{c}^{\nu_{t}}}{2}\right)^{2} \mathrm{e}^{\nu_{t}} \underset{h \downarrow 0}{\longrightarrow} 0
\end{aligned}
$$

$$
\begin{aligned}
\mathrm{E}\left[h^{-1}\left(\nu_{t+h}-\nu_{l}\right)^{4} \mid \mathcal{M}_{t}\right]=h^{3} \beta^{4}\left(\alpha-\nu_{t}\right)+ & h^{2} \beta^{2} \gamma^{2}\left(\alpha-\nu_{t}\right)^{2}+4 h^{2} \beta^{2}\left(\alpha-\nu_{l}\right)^{2} \\
& +h^{2} \beta^{2} \gamma^{2}\left(\alpha-\nu_{l}\right)^{2}+3 h \gamma^{4} \underset{h 10}{\longrightarrow} 0 .
\end{aligned}
$$

A hipótese 4 é então satisfeita com $\delta=2$

Ainda resta provar que o processo $\left\{X_{l}\right\}$ tem solução fraca única. O traballıo Nelson (1991) rosume algumas condiçôs suficientes para a existência e unicidade de um processo de difusão limite. Tomenos como exemplo o caso cnunciado a seguir.

Proposição 2.11. Consideremos à notaçä́o aprescutada no teorema 2.10. Assegurando que $\mu_{h}(x)$ e $\Sigma_{h}(x)$ sejam funções contínuas tanto em $x$ quanto en $h$ e lambém garantindo que as derivadas parciais de primeira e segunda ordens com relação a $x$ sejam contínuas, teremos definido unicamente um processo de difusão limite.

P'ela equação (2.14) temos que $\mu_{h}$ é contínua tanto em $H_{t}$ como $\mathrm{en}_{\nu} \nu_{t}$, as derivadas de primeira e segunda ordens são nulas e, portanto, contínuas. As derivadas parciais com rclação a $\nu_{t}$ também são contínuas como podemos notar

$$
\frac{\partial \mu_{t}}{\partial \nu_{t}}\left(\left(H_{t}, \nu_{t}\right)\right)=\left(\begin{array}{c}
-\mathrm{e}^{\nu_{t}} / 2 \\
-\beta
\end{array}\right)
$$

$$
\frac{\partial^{2} \mu \mu_{l}}{\partial \nu_{l}^{2}}\left(\left(H_{t}, \nu_{t}\right)\right)=\left(\begin{array}{c}
-\mathrm{e}^{\nu_{l}} / 2 \\
0
\end{array}\right) .
$$

A contimuidade da função $\Sigma_{h}$ nats duas varrávcis ć facihnente notada pela cquação (2.18). $\Lambda s$ derivarlas parciais con relação a $H_{l}$ e a $\nu_{\iota}$ são iguais a zero. Já as derivadas parciais com relação a $\nu_{t}$ são datalas por

$$
\frac{\partial \Sigma_{L_{l}}}{\partial \nu_{t}}\left(\left(I_{t}, \nu_{t}\right)\right)=\left(\begin{array}{cc}
\mathrm{e}^{\nu_{t}} & \frac{\gamma \rho}{2} \mathrm{e}^{\nu_{t} / 2} \\
\frac{\gamma \rho}{2} \mathrm{e}^{\nu_{t} / 2} & 0
\end{array}\right)
$$




$$
\frac{\partial^{2} \Sigma_{h}}{\partial \nu_{t}^{2}}\left(\left(H_{t}, \nu_{t}\right)\right)=\left(\begin{array}{cc}
\mathrm{e}^{\nu_{t}} & \frac{\gamma \rho}{4} \mathrm{e}^{\nu_{t} / 2} \\
\frac{\gamma \rho}{4} \mathrm{e}^{\nu_{t} / 2} & 0
\end{array}\right),
$$

e portanto são contímuas. Isto implica a micidarle da solução.

Finalmente podemos conchuir que (2.11)-(2.13), conhecida como aproximasão de Fuler do modelo I, converge fracamente para o processo de difusão definido por (2.8)-(2.10), quc nada mais é do que o modelo I na sua forma matricial.

No entanto essa aproximação de Euler não é un processo ARCH. Para termos uma aproximação ARCII para o processo de difusão em cuestão, substituímos a ecquação (2.12) do sistcma discreto por

$$
\nu_{i+h}=\nu_{t}+h \beta\left(\alpha-\nu_{t}\right)+h^{1 / 2} g\left(Z_{\ell+h}\right)
$$

em que $g(\cdot)$ é uma funçĩo mensurável com $\mathrm{E}\left[\left|g\left(Z_{t}\right)\right|^{2+\delta}\right]<\infty$ para algun $\delta>0 \mathrm{e}$

$$
\operatorname{Var}\left(\begin{array}{c}
Z_{i} \\
g\left(Z_{t}\right)
\end{array}\right)=\left(\begin{array}{ll}
1 & \rho \\
\rho & 1
\end{array}\right)
$$

Para que o sistema definido pelias equacuos (2.11), (2.25) e (2.26) correspondia a um processo EGARCH, como feito cm Bollerslev, Fngle \& Nelson (1994) e em Nelson (1990a), consideramos a fumçăo $g(\cdot)$ como

$$
g\left(Z_{i}\right)=\rho \gamma Z_{t}+\gamma\left(\frac{1-\rho^{2}}{1-2 / \pi}\right)^{1 / 2}\left[\left|Z_{t}\right|-\left(\frac{2}{\pi}\right)^{1 / 2}\right],
$$

já que $\mathrm{E}\left(\left|Z_{i}\right|\right)=(2 / \pi)^{1 / 2}, \mathrm{E}\left(Z_{i}\left|Z_{i}\right|\right)=0$ e $\operatorname{Var}\left(\left|Z_{i}\right|\right)=1-2 / \pi$. Este morlelo rliscreto á valorizado pois incorpora as propricdades dos modelos $\Lambda \mathrm{RCH}$, como a lácil obtenção dáa verossimilhança e sua iıferência. Neste trabalho cufocaremos no capítulo 4 uma outra forma de inferência, que não utiliza a aproximação ARCH, e sim a aproximação de Euler.

\subsubsection{Modelo II}

Chamaremos de modelo II o processo de difusão descrito a segnir

$$
\begin{aligned}
& d H_{l}=\left(\mu-\frac{\sigma_{l}^{2}}{2}\right) d l+\sigma_{t} d W_{l} \\
& d \sigma_{l}^{2}=\delta\left(\lambda-\sigma_{l}^{2}\right) d t+\xi \sigma_{t} d \widetilde{W}_{l},
\end{aligned}
$$




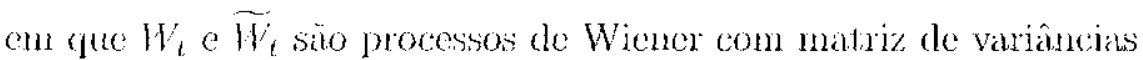

$$
\operatorname{Var}\left(\begin{array}{l}
W_{t} \\
\widetilde{W}_{t}
\end{array}\right)=\left(\begin{array}{ll}
1 & \rho \\
\rho & 1
\end{array}\right) .
$$

Scja o processo a tempo discreto dado por

$$
\begin{aligned}
& H_{t+h}=H_{l}+h\left(\mu-\frac{\sigma_{l}^{2}}{2}\right)+h^{1 / 2} \sigma_{l} Z_{t, h} \\
& \sigma_{l+h}^{2}=\sigma_{t}^{2}+h \delta\left(\lambda-\sigma_{l}^{2}\right)+h^{1 / 2} \xi \sigma_{t} \widetilde{Z}_{t \mid h},
\end{aligned}
$$

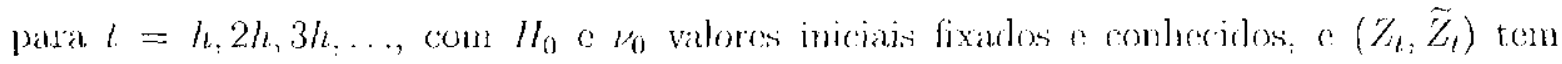

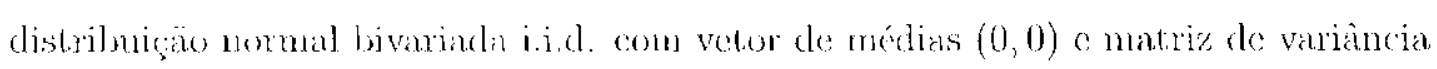

$$
\operatorname{Var}\left(\begin{array}{l}
z_{i} \\
\tilde{Z}_{l}
\end{array}\right)=\left(\begin{array}{ll}
1 & \rho \\
\rho & 1
\end{array}\right)
$$

Sejanu us proceshos contumus no tompo $H_{\tau}^{(h)} \mathrm{e} \sigma_{\tau}^{2(h)}$ definidos por

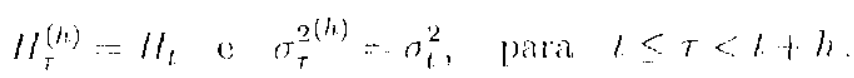

Análogo à segäo anterior, $\mathcal{M}_{t}$ denota a $\sigma$-álgchra gerala por $\left\{H_{\tau}, \sigma_{\tau}^{2}, 0 \leq \tau \leq l\right\}$. Desta forma

$$
\begin{aligned}
h^{-1} \mathrm{E}\left[\left(\begin{array}{c}
H_{t-1}-H_{t} \\
\sigma_{t+h}^{2}-\sigma_{t}^{2}
\end{array}\right) \mid \mathcal{M}_{i}\right] & =h^{-1} \mathrm{E}\left[\left(\begin{array}{c}
h\left(\mu-\frac{\sigma_{t}^{2}}{2}\right)+h^{1 / 2} \sigma_{t}^{2} Z_{t+h} \\
h \delta\left(\lambda-\sigma_{t}^{2}\right)+h^{1 / 2} \xi \sigma_{t} \widetilde{Z}_{t+h}
\end{array}\right) \mid \mathcal{M}_{t}\right] \\
& =h^{-1}\left[\left(\begin{array}{c}
h\left(\mu-\frac{\sigma_{t}^{2}}{2}\right) \\
h \delta\left(\lambda-\sigma_{l}^{2}\right)
\end{array}\right) \mid \mathcal{M}_{t}\right]=\left(\begin{array}{c}
\mu-\frac{\sigma_{t}^{2}}{2} \\
\delta\left(\lambda-\sigma_{t}^{2}\right)
\end{array}\right) .
\end{aligned}
$$

Vanos agora calcular a matriz de varinucia por midade de lompo,

$$
\begin{aligned}
h^{-1} \operatorname{Var}\left[\left(\begin{array}{c}
H_{t+h} \cdots \\
\sigma_{t+h}^{2}-\sigma_{t}^{2}
\end{array}\right) \mid \mathcal{M}_{t}\right] & =h^{-1} \operatorname{Var}\left[\left(\begin{array}{c}
h^{1 / 2} \sigma_{t} Z_{t+h} \\
h^{1 / 2} \xi \sigma_{t} \tilde{Z}_{t+h}
\end{array}\right) \mid \mathcal{M}_{t}\right] \\
\operatorname{Var}\left[\left(\begin{array}{cc}
\sigma_{t} & 0 \\
0 & \xi \sigma_{t}
\end{array}\right)\left(\begin{array}{c}
Z_{t} \\
\widetilde{Z}_{t}
\end{array}\right) \mid \mathcal{M}_{l}\right] & =\left(\begin{array}{cc}
\sigma_{t} & 0 \\
0 & \xi \sigma_{t}
\end{array}\right) \operatorname{Var}\left(\begin{array}{c}
Z_{t} \\
\widetilde{Z}_{t}
\end{array}\right)\left(\begin{array}{cc}
\sigma_{t} & 0 \\
0 & \xi \sigma_{t}
\end{array}\right)^{\prime} \\
& =\left(\begin{array}{cc}
\sigma_{t}^{2} & \xi \rho \sigma_{t}^{2} \\
\xi \rho \sigma_{t}^{2} & \xi^{2} \sigma_{t}^{2}
\end{array}\right) .
\end{aligned}
$$




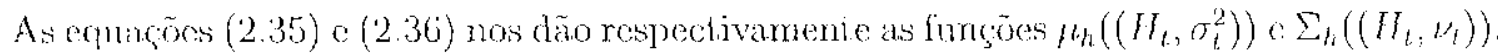
Novanente temos que a tendencia o a matriz de difusão năo depondem de h o então temos

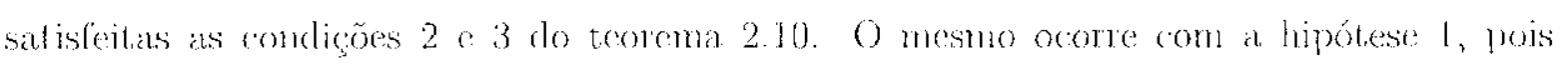

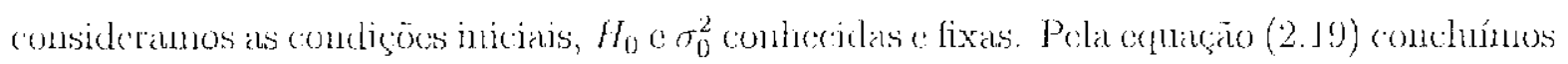
cliretamente que

$$
\begin{aligned}
E\left[h^{\cdots}\left(h_{h+h}-h_{l}\right)^{4} \mid \mathcal{M}_{l}\right]=h^{3}\left(\mu-\frac{\sigma_{i}^{2}}{2}\right)^{4} & +4 h^{2}\left(\mu \cdots \frac{\sigma_{l}^{2}}{2}\right) \sigma_{l}^{2}+3 h \sigma_{l}^{4} \\
& +2 h^{2}\left(\mu-\frac{\sigma_{l}^{2}}{2}\right)^{2} \sigma_{l}^{2} \rightarrow 0
\end{aligned}
$$

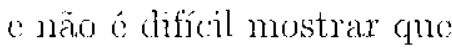

$$
\begin{aligned}
& \left.E_{i}^{[} h^{-1}\left(\sigma_{i-1}^{2}-\sigma_{l}^{2}\right)^{4} \mid \mathcal{M}_{t}\right]=h^{3} \delta^{1}\left(\lambda-\sigma_{i}^{2}\right)+1 h^{2} t^{2} \xi^{2}\left(\lambda-\sigma_{i}^{2}\right)^{2}+3 h \xi^{4} \sigma_{i}^{4} \\
& +2 h^{2} \partial^{2} \xi^{2}\left(\lambda-\sigma_{l}^{2}\right)^{2} \underset{h 10}{\longrightarrow} 0
\end{aligned}
$$

Logo, para $\delta--2$, a hipótose 4 é süt isfeilat

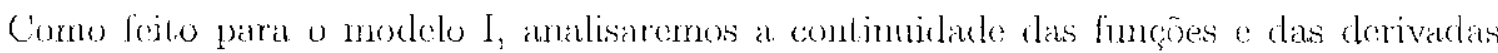

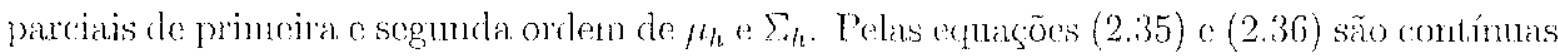

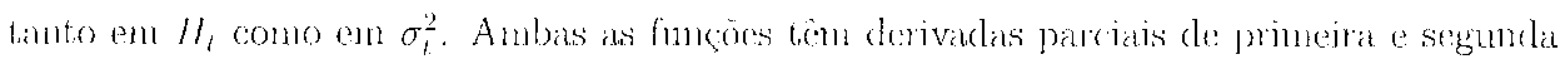

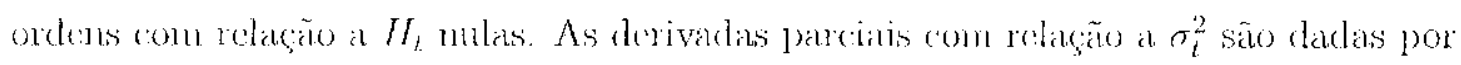

$$
\begin{array}{ll}
\frac{\partial \mu_{h}}{\partial \sigma_{l}^{2}}\left(\left(H_{l}, \sigma_{l}^{2}\right)\right)=\left(\begin{array}{c}
-1 / 2 \\
-\delta
\end{array}\right), & \frac{\partial^{2} \mu_{h}}{\partial\left(\sigma_{l}^{2}\right)^{2}}\left(\left(H_{l}, \sigma_{l}^{2}\right)\right)=\left(\begin{array}{l}
0 \\
0
\end{array}\right) \\
\frac{\partial \Sigma_{h}}{\partial \sigma_{l}^{2}}\left(\left(H_{l,}, \sigma_{l}^{2}\right)\right)=\left(\begin{array}{cc}
1 & \xi \rho \\
\xi \rho & \xi^{2}
\end{array}\right) \text { e } & \frac{\partial^{2} \Sigma_{h}}{\partial\left(\sigma_{l}^{2}\right)^{2}}\left(\left(I_{l}, \sigma_{l}^{2}\right)\right)=\left(\begin{array}{ll}
0 & 0 \\
0 & 0
\end{array}\right)
\end{array}
$$

De acordo com a proposicüo 2.11 fica provadia a muicidade da solução.

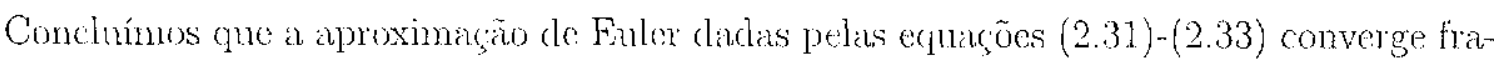

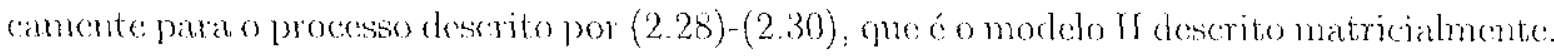

\subsection{Conhecendo os Modelos}

() sistema descrito en (1.13) atende dis hipóteses em que so basciann mu modelo de

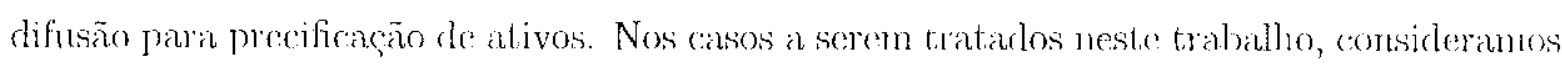


a. funçu $\mu_{S}$ constidnte e ignal a $\mu$, a a função $\sigma_{S}$ igual a volatilirlade no tempo $t$, $\sigma_{t}$ obtendo a segnimte coplagano

$$
d S_{l}=\mu S_{l} d t+\sigma_{1} S_{l} d l V_{l}
$$

en que Wr é un movimento browninuo. Isso significa que a taxa de retono esperarla contimamente ajustada é comsiderada proporcional ao valor passado do ativo, e que a variancia condicional do retomo é dada pela volatilidade $n 0$ tempo a que o retomo a conelicionaco. veja lijuóteses (1.1) e (1.2).

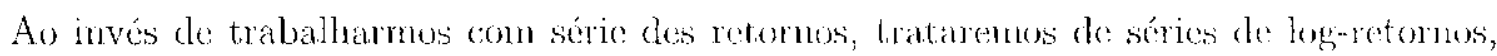

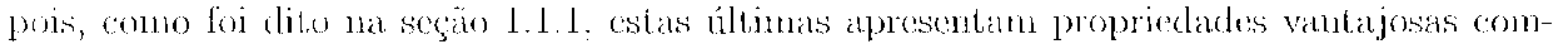

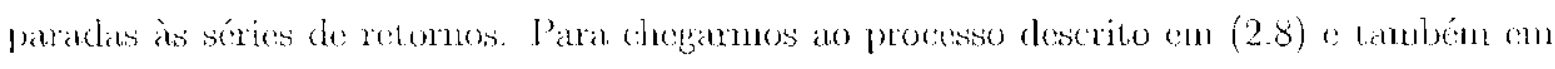

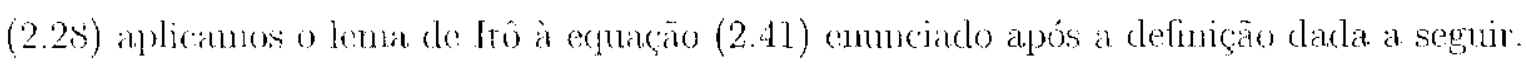

Dofinição 2.12 (Processo de ltô). Chammunos de processo de Itô mu processio $X_{t}$ defindo no espago de probabiliclike $(\Omega, \mathcal{B}, P)$ que satisfaça a equaço diferencial

$$
d X_{l}=a\left(l, X_{l}\right) d l+b\left(l, X_{l}\right) d l V_{t}, \quad \forall \omega \in \Omega
$$

em que $\sqrt{|a|} \mid$ eb pertencem aro espano $l^{2}$

Lema 2.13 (Lema de Itô). Sejan $X_{t}$ um processo de Itô e $f:[0,7] \times \mathbb{R} \rightarrow \mathbb{R}$ mma funcrio

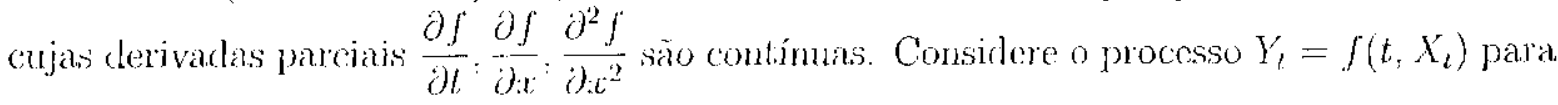
$0 \leq 1 \leq T$. Entio

$$
\begin{aligned}
& d f\left(l, X_{l}\right)=\left[\frac{\partial j}{\partial l}\left(l, X_{l}\right)+a\left(l, X_{t}\right) \frac{\partial f}{\partial t}\left(l, X_{t}\right): \frac{l}{2} b^{2}\left(l, X_{t}\right) \frac{\partial^{2} J}{\partial x^{2}}\left(l, X_{l}\right)\right] d t \\
& +b\left(t, X_{l}\right) \frac{\partial f}{\partial t}\left(l, X_{l}\right) d W_{t}
\end{aligned}
$$

com probalsiliclacke 1 para $0 \leq t \leq T$. (veja p.ex. Oksendal, 1998, 1). 14).

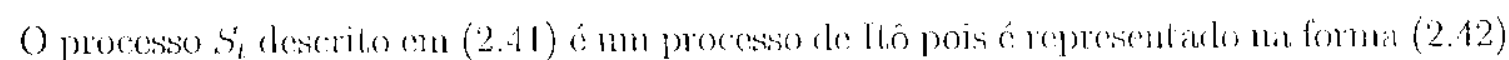
com

$$
a\left(t, S_{t}\right)=\mu S_{i} \quad \text { e } b\left(L, S_{t}\right)=\sigma_{l} S_{i}
$$

Seja, $/ / \equiv$ hn S. Tomanulo $f(1, x)=$ In $x$ temos

$$
\frac{\partial f}{\partial x}(l, x)=0, \quad \frac{\partial f}{\partial x}(l, x)=\frac{1}{x^{2}}, \quad \frac{\partial^{2} f}{\partial x^{2}}(l, x)=-\frac{1}{x^{2}}
$$




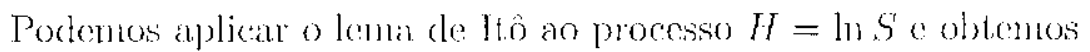

$$
d H_{l} \cdots\left[\mu S_{t} \frac{1}{S_{t}}+\frac{1}{2}\left(\sigma_{i} S_{i}\right)^{2}\left(-\frac{1}{S_{i}}\right)\right] d t+\sigma_{i} S_{t} \frac{1}{S_{i}} d W_{t}
$$

Finalmente

$$
d H_{l} \cdot\left(\mu \frac{\sigma_{i}^{2}}{2}\right) d \omega \cdot+\sigma_{l} d W_{t}
$$

Usanos dois tipos de modelos, un que desereve a log-volatilitade e ontro que dencrevo

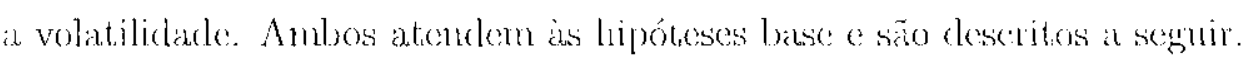

\subsubsection{Modelo I}

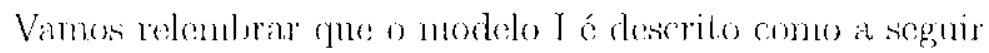

$$
\begin{aligned}
& d H_{t}=\left(\mu-\frac{e^{p_{t}}}{2}\right) d l \cdot+c^{\nu_{t} / 2} d H_{t} \\
& d w_{1+h}-\beta\left(\alpha-\nu_{1}\right) d u+\gamma d \hat{W}_{1}
\end{aligned}
$$

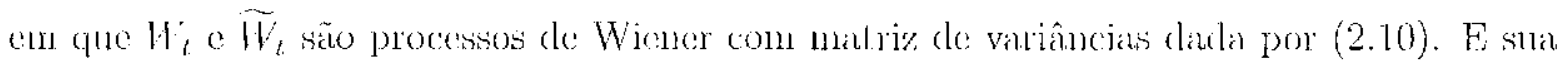
aproximatano de Fuler ó dada por

$$
\begin{aligned}
& H_{t+h}=H_{t}+h\left(\mu-\frac{\mathrm{e}^{L_{t}}}{2}\right)+1 \cdot h^{1 / 2} \mathrm{e}^{h_{+} / 2} Z_{t+h} \\
& \nu_{t+h}=\nu_{t}+h \beta\left(\omega \cdot \mu_{h}\right)+h^{1 / 2} \gamma \tilde{Z}_{t+h} \text {, }
\end{aligned}
$$

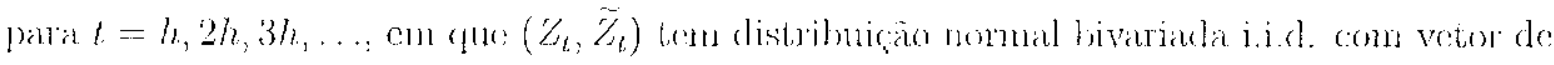

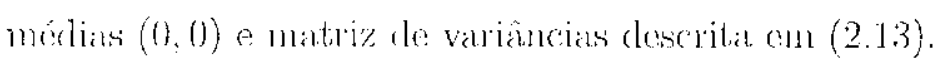

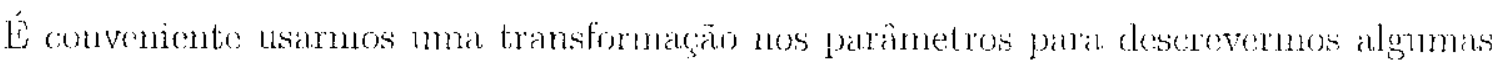

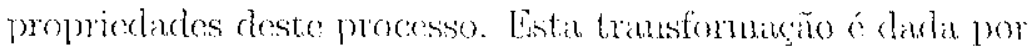

$$
\sigma^{*}=\exp \{\alpha / 2\} \quad \text { e } \quad \phi:=1-\beta
$$

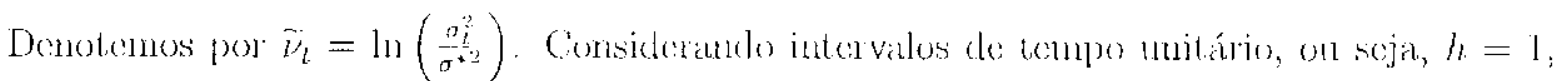
temos que o morlelo descrito jor (2.11) a (2.12) equivale ano modelo a segur

$$
\begin{aligned}
& H_{t+1}=H_{t}+\left(11 \cdots \sigma^{* 2} \frac{\mathrm{e}^{\tilde{j}_{t}}}{2}\right)+\sigma^{*} \mathrm{e}^{\tilde{i}_{t} / 2} Z_{t+1} \\
& \ddot{\nu}_{l 11}=\omega \ddot{l}_{t}+\gamma \dddot{Z}_{l-1}
\end{aligned}
$$




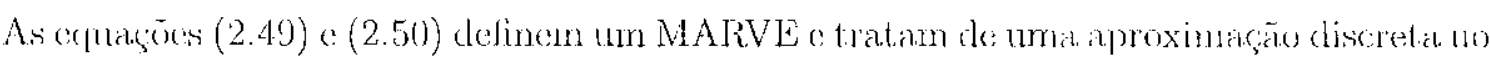
bempo do jrocesso de Ornstein-Uhlenbeck, um processo de difusáto combínno no tempo usado

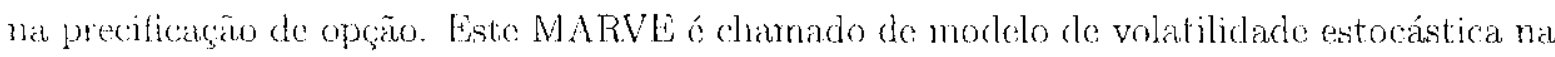

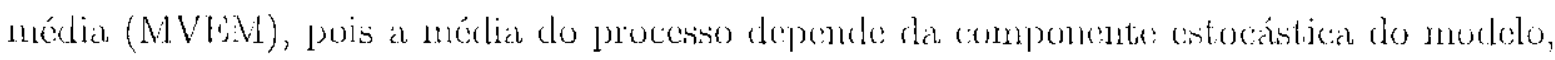

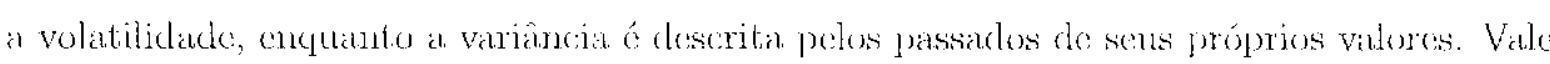

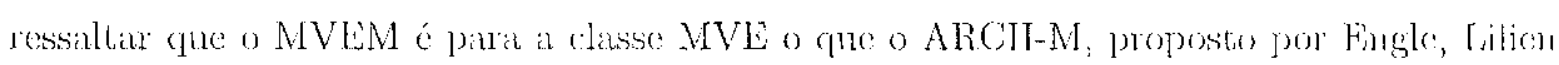
\& Robins (1987), o paral a clatise ARCH

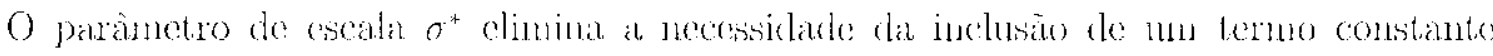

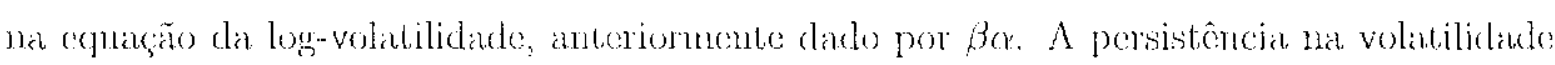
passada ó deserita pelo parâmetro $\phi$. Para tomos um processo estacionário $\phi$ dove sel

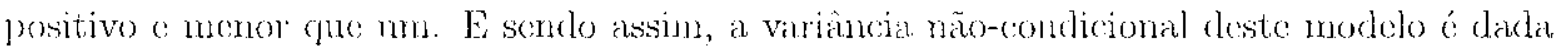
1)OI:

$$
\sigma^{22} \operatorname{cxp}\left(0.5 \frac{\gamma^{2}}{1-\phi^{2}}\right) .
$$

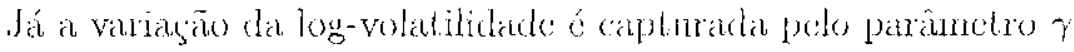

O traballo lioopman \& Uspensty (2002) compara mn MARVE mais geral do yue o

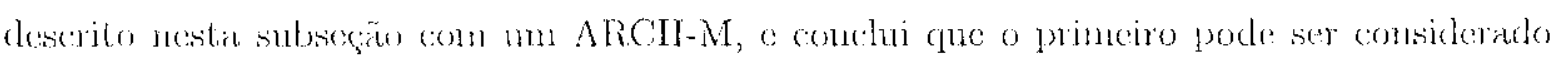

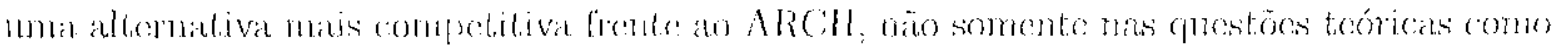

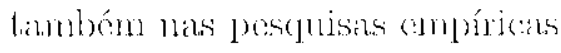

\subsubsection{Modelo II}

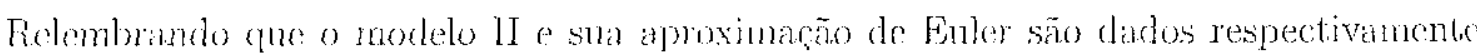
pol

$$
\begin{aligned}
& d I_{t}=\left(\mu-\frac{\sigma_{l}^{2}}{2}\right) d l+\sigma_{t} d W_{i} \\
& d \sigma_{l}^{2}-\delta\left(\lambda-\sigma_{t}^{2}\right) d l-i \xi \sigma_{i} d \widetilde{W}
\end{aligned}
$$

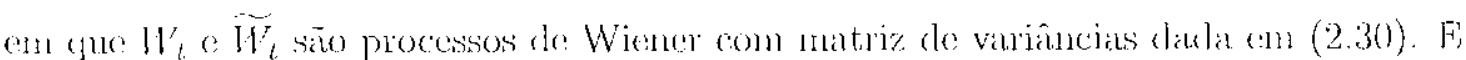

$$
\begin{aligned}
& H_{i+1}=H_{t}+\left(\mu-\frac{\sigma_{l}^{2}}{2}\right)+\sigma_{i} Z_{i+1} \\
& \sigma_{t+1}^{2}=\sigma_{i}^{2}+\delta\left(\lambda-\sigma_{i}^{2}\right)+\xi \sigma_{i} \ddot{Z}_{t+1},
\end{aligned}
$$

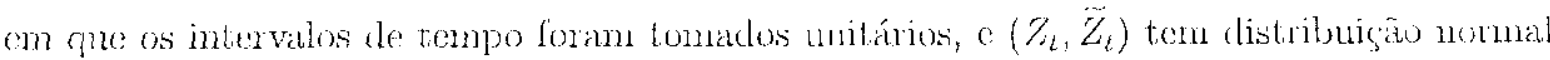

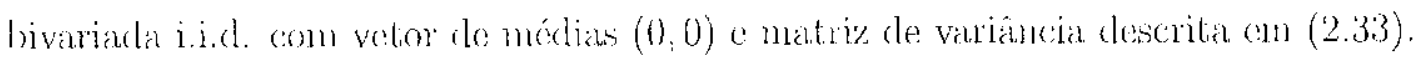




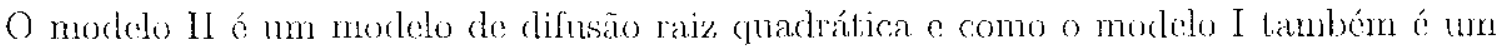
MVEM. Nole a persistêneia na volatiliclade passada ó descrita pelo parânetro $\varphi \doteq 1-\delta$. Para termos um processo estarionário $\varphi$ reve ser positivo e menor que $111 n$.

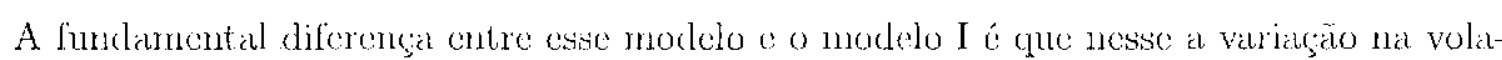
tilicale é heterocedática, ignal a $\xi^{2} \sigma_{t}^{2}$.

\subsection{Conclusão}

Os teoremas que provan a convergentia da aproxinaçäo de Fuler têm como hipótese as condiçös globais de Jipschitz, (veja Nelson, 1990a). Fssas condicões não tão satisfejtà pelos

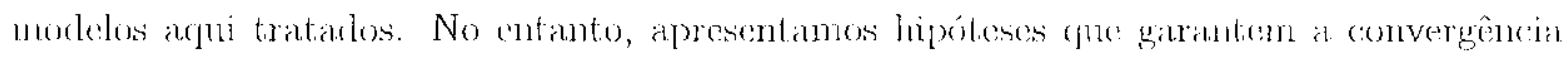
fraca de cortos processos; o mostranos que estas săo satisfeitas pelas aproximasoes de Euler dos modelos 1 e II. Portadn ao tratamos dos modelos discretizados, não estanos, pon completo, abandonando as pronissas estabelecidas pela modelagen contínula. Alén disso,

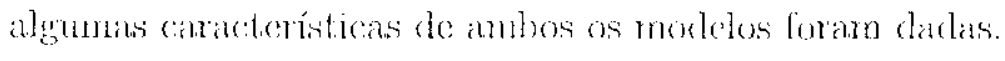




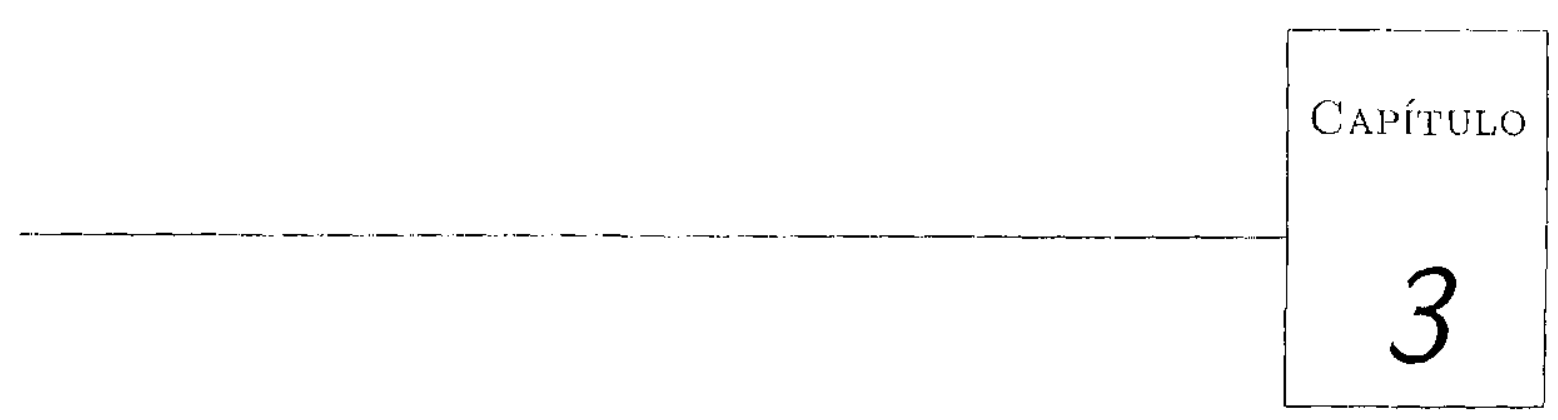

Modelo Linear

A segnir apresentaromos considerações preliminares e depois darennos à icléia básica do

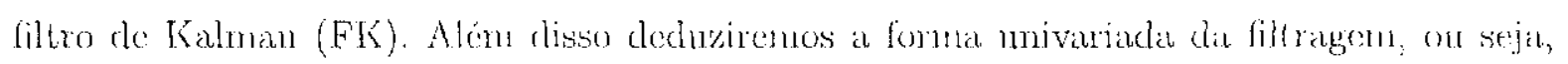

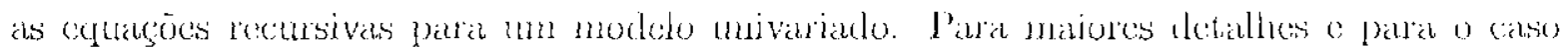
multivariado reconemelanuos Harvey (1989) on Davis \&. Vinter (1985).

\section{$3.1 \quad$ Filtro de Kalman}

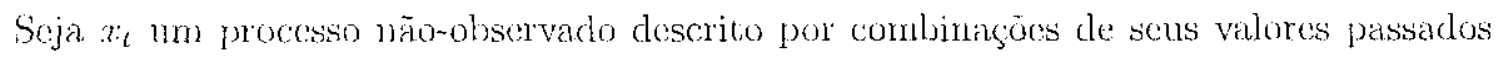
acrescido de mma componente aleatória. E seja yt um processo observado descrito por w

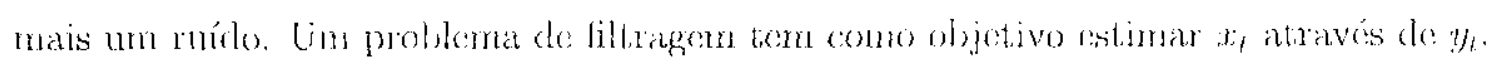

O FK é um algoritno recursivo que objetiva calcular o estimalor ótimo do estado tre no

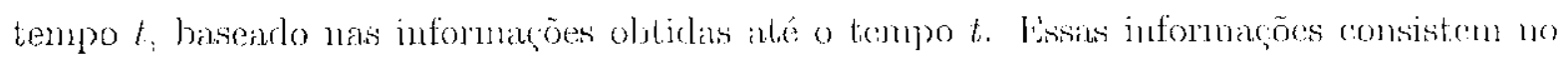


conjumto de observações $y_{t}-\left(y_{0}, y_{1}, \ldots, y_{t}\right)$. () modelo é descrito por

$$
\begin{array}{ll}
y_{t}=a_{t} x_{t}+b_{t}+\varepsilon_{t} & \text { (observaciano) } \\
a_{t} \cdot a_{t}+1+d_{t}+\eta_{t} \eta_{t} & \text { (estarlo), }
\end{array}
$$

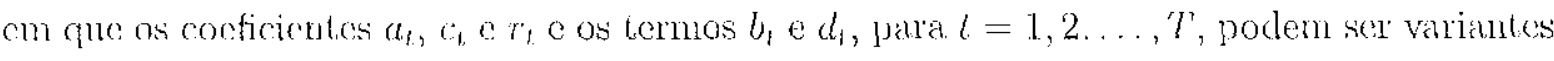

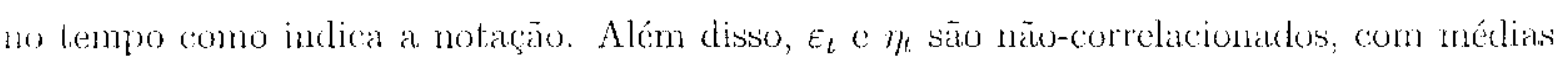
zoro e variatucias igunis a $\sigma_{c}^{2}$ o $\sigma_{\eta}^{\prime \prime}$, respectivamente, on seja,

$$
E\left(\varepsilon_{1} \eta_{s}\right)=0 \quad \forall s, l-0,1 \ldots, T
$$

$$
\operatorname{Var}\left(\varepsilon_{l}\right)-\sigma_{z}^{2} \quad \text { c } \operatorname{Var}\left(\eta_{t}\right)=\sigma_{\eta}^{2}
$$

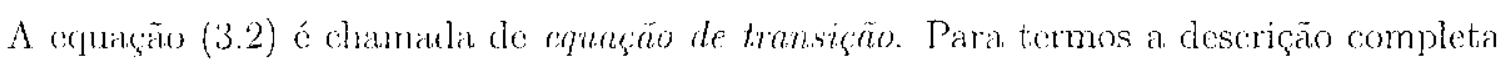
do modelo, revernos combece as condicón iniciais do sistema e assumir que as perturbaçoes

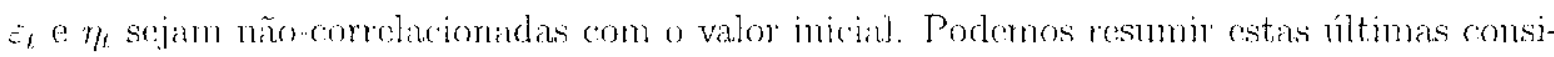
delingoes da sogninto forma.

$$
E\left(x_{0}\right) \cdots \bar{x}_{0} \text { o } \operatorname{Var}\left(x_{0}\right)=P_{0}, \quad \vec{x}_{0} \text { e } P_{0} \text { conhocirios, }
$$

$$
E\left(\eta_{1} x_{0}\right)=0 \quad \& \quad E\left(\varepsilon_{1}, x_{0}\right)-0 \quad \forall l-0,1, \ldots, T .
$$

\subsubsection{Obtendo o Filtro de Kalman}

() FK provem de ma propriedade da distribucão nomal, tal propriedade perrute

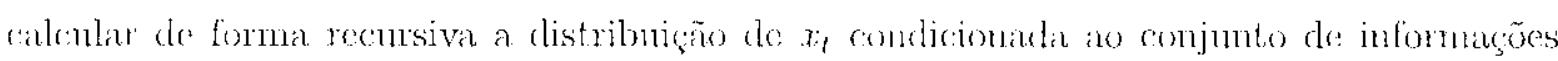
obtidas até o tempo $l, V 1 \leq l \leq T$. Essas distributéos condicionajs são gaussianas e

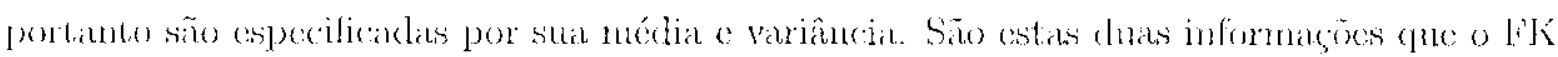
obtém como veremos a seguir

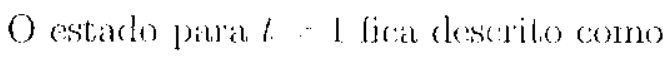

$$
x_{1}=c_{1} x_{0}+d_{1}+r_{1} r_{1} .
$$

Logo $x_{1}$ é una conbinagão lincal de duas variáveis normais independentes. Então ó taumbúm mora viuível normal com média condicional

$$
\bar{x}_{1 \mid 0}-a_{1} \bar{x}_{0}+d_{1}
$$


e vatumcin conticional

$$
\Gamma_{j \mid 0}=c_{1}^{2} P_{0}+r_{1}^{2} \sigma_{1}^{2}
$$

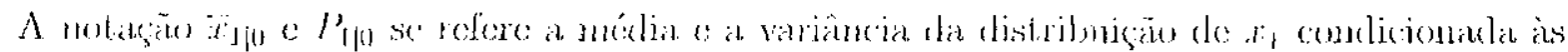
i)formatoses emol- 0 .

Querenos a clist ribuiçäo de $x_{1} \mid y_{1}$. Para isso escrevenos

$$
\begin{gathered}
x_{1}=\bar{x}_{100}+\left(x_{1}-\bar{x}_{100}\right) \\
y_{1}=a_{1} \bar{x}_{1,0}+a_{1}\left(x_{1}-\bar{x}_{10}\right)+b_{1}+z_{1} .
\end{gathered}
$$

F. enlizo temos que o vetor $\left(\begin{array}{ll}x_{1} & y_{1}\end{array}\right)$ tem distribuiçäo conjunta normal bivariada com vetor

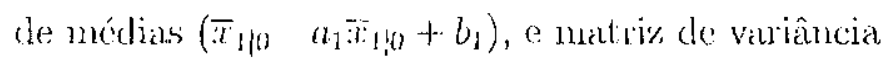

$$
\left(\begin{array}{cc}
P_{1 \mid 0} & a_{1} P_{1 \mid 0} \\
a_{1} P_{t \mid a 1} & a_{1}^{2} P_{1 \mid 0+}+\sigma_{c}^{2}
\end{array}\right)
$$

Lema 3.1. Se $(x \quad y)$ hem distribriçăo normed conjumb bivariada conn vetor de médias

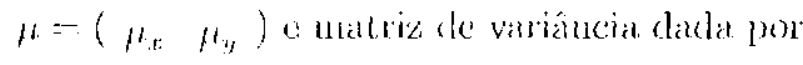

$$
\Sigma=\left(\begin{array}{cc}
\sigma_{x}^{2} & \sigma_{x y}^{2} \\
\sigma_{x y}^{2} & \sigma_{y}^{2}
\end{array}\right)
$$

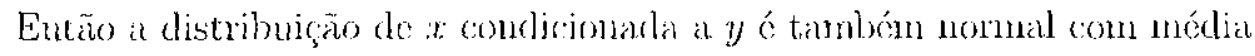

$$
\mu_{1: j] y}=\mu_{x}+\sigma_{x y}^{2}\left(\sigma_{y}^{2}\right)^{-1}\left(y-\mu_{y}\right)
$$

e variaincia

$$
\sigma_{x \mid y}^{2}=\sigma_{x}^{2}-\left(\sigma_{x y}^{2}\right)^{2}\left(\sigma_{y y}^{2}\right)^{-1} .
$$

Prova. Por hipótese temos que

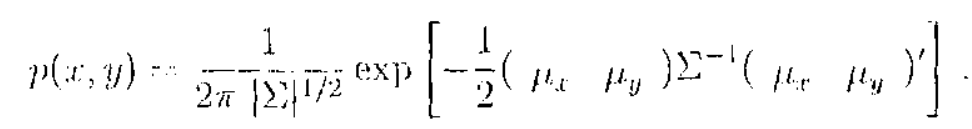

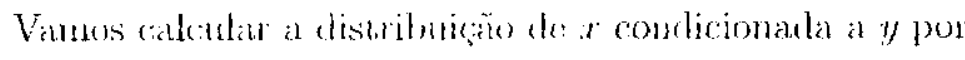

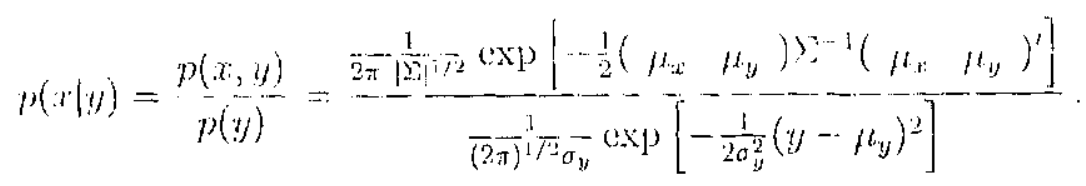


É conveniente utilizar a seguinte fómula

$$
\left(\begin{array}{cc}
1 & -\sigma_{x y}^{2}\left(\sigma_{y}^{2}\right)^{-1} \\
0 & 1
\end{array}\right) \Sigma\left(\begin{array}{cc}
1 & 0 \\
-\sigma_{x y}^{2}\left(\sigma_{y}^{2}\right)^{-1} & 1
\end{array}\right)=\left(\begin{array}{cc}
\sigma_{x}^{2}-\left(\sigma_{x y y}^{2}\right)^{2}\left(\sigma_{y}^{2}\right)^{-1} & 0 \\
0 & \sigma_{y}^{2}
\end{array}\right)
$$

pois facilita conchuir glue

$$
\Sigma^{-1}=\left(\begin{array}{cc}
1 & 0 \\
-\sigma_{x y y}^{2}\left(\sigma_{y y}^{2}\right)^{-1} & 1
\end{array}\right)\left(\begin{array}{cc}
\sigma_{x:}^{2}-\left(\sigma_{x y y}^{2}\right)^{2}\left(\sigma_{y}^{2}\right)^{-1} & 0 \\
0 & \sigma_{y}^{2}
\end{array}\right)\left(\begin{array}{cc}
1 & -\sigma_{x y y}^{2}\left(\sigma_{y y}^{2}\right)^{-1} \\
0 & 1
\end{array}\right)
$$

Substituindo esta última informação $\mathrm{cm}$

$$
\left(\mu_{x:} \mu_{y}\right) \Sigma^{-1}\left(\begin{array}{ll}
\mu_{x} & \mu_{y}
\end{array}\right)^{\prime}
$$

e demotauklo por $A=\sigma_{x-3}^{2}-\left(\sigma_{x y y}^{2}\right)^{2}\left(\sigma_{y}^{2}\right)^{-1}$ (llegamos a

$$
\begin{array}{r}
\left(x-\mu_{x}\right)^{2} A-2\left(x-\mu_{x}\right)\left(y-\mu_{y y}\right) \sigma_{x y}^{2}\left(\sigma_{i y}{ }^{1}\right) A+\left(y-\mu_{y}\right) \sigma_{x y y}^{2}\left(\sigma_{y}^{-1}\right)^{2} A+\left(y-\mu_{y}\right)^{2}\left(\sigma_{y}^{-1}\right) \\
=A\left[x-\left(\mu_{x}-\left(y-\mu_{y}\right) \sigma_{x y}^{2}\left(\sigma_{y y}^{-1}\right)\right)\right]^{2}+\left(y-\mu_{y}\right)^{2}\left(\sigma_{y}{ }^{1}\right) .
\end{array}
$$

Finaluentic

$$
p(x \mid y)=\frac{1}{\sqrt{2 \pi \sigma_{x \mid y}^{2}}} \exp \left[\frac{1}{2 \sigma_{x ; y}^{2}}\left(x-\mu_{x: y}\right)^{2}\right]
$$

em que $\mu_{x \mid y}$ e $\sigma_{x \mid y}^{2}$ são como nas equacǒss (3.14) e (3.15), respectivamente.

Pelo loma 3.1 comeluúnus entiano gule?

$$
x_{1} \mid y_{1} \sim \mathcal{N}\left(\bar{x}_{1}, P_{1}\right)
$$

all glue

$$
\begin{gathered}
\bar{x}_{1}-\bar{x}_{100} \mid a_{1} P_{1 \mid 0} f_{1}^{-1}\left(y_{1}-a_{1} \bar{x}_{10}-b_{1}\right) . \\
P_{1}=P_{1 \mid 0}-a_{1}^{2} P_{1 \mid 0}^{2} f_{1}^{-1}
\end{gathered}
$$

e

$$
\int_{1}: a_{1}^{2} P_{1+0}+\sigma_{c}^{2}
$$

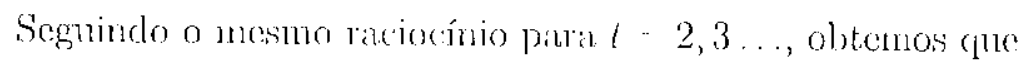

$$
\bar{x}_{i \mid t-1}=c_{l} \bar{x}_{l \ldots 1}+d_{l}
$$




$$
P_{i, k+1}=\cdots c_{i}^{2} P_{i-1}+r_{i}^{2} \sigma_{n}^{2}
$$

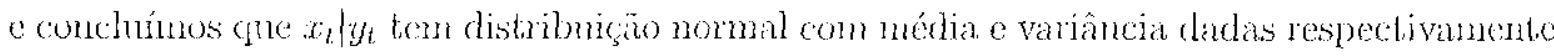
jor

$$
\bar{x}_{l}=\bar{x}_{t \mid l-1}+u_{l}^{2} P_{t \mid 1-1} f_{l}^{-1}\left(y_{t}-a_{t} \bar{x}_{t \mid l-1}-b_{l}\right)
$$

$$
P_{t}-P_{t \mid l-1}-a_{t}^{2} P_{t \mid t}^{2} I_{i}^{-1}
$$

anc clie:

$$
f_{t}=a_{l}^{2} I_{i \mid t}+1 \cdot \sigma_{\varepsilon}^{2}
$$

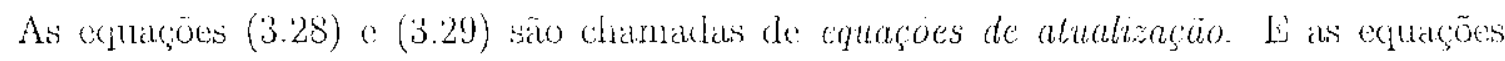
(3.26) e (3.27) são as equasous de prediça do FK.

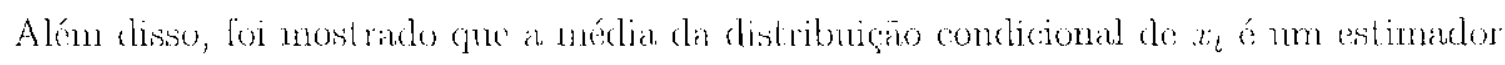

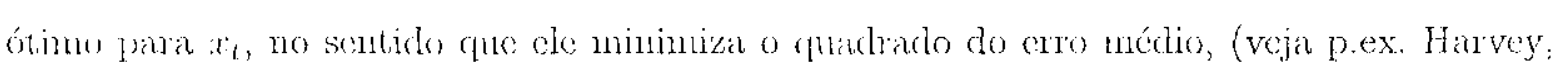
$3989)$.

\subsection{Modelo Invariante no Tempo}

Considerenos o modelo discreto dato por (2.11)-(2.13) com $h=1$ (2p=0. Aproximemos

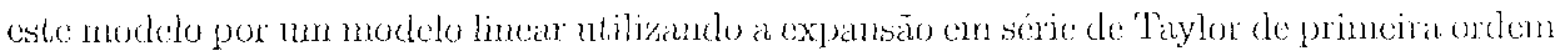

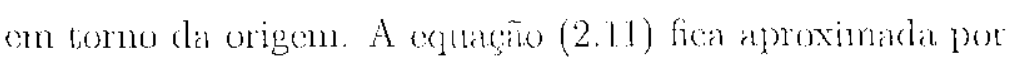

$$
H_{t+1}=H_{t}+\mu-\frac{1}{2}+\left(\frac{1}{2}+\frac{1}{2} Z_{t-1}\right) \nu_{t}+\frac{1}{2} Z_{t+1} .
$$

Este processe é comblicionalunente ganssiano. Para simplificá-lo, considerames clue o termo $1 / 2-Z+-1 / 2$ é constante igual a a, obtendo cue

$$
H_{t+1}=H_{t}+\mu-\frac{1}{2}+\left(n \mu_{t}+z_{t}\right.
$$

an que $\varepsilon_{1} \sim \mathcal{N}(0,1 / 4)$.

Sejam

$$
b-\mu-\frac{1}{2}, \quad c=1-\beta, \quad d=\beta \alpha \quad \text { o } \quad \gamma=\gamma^{2} .
$$


Além clisso, tomando

$$
y_{t-1}-H_{t+1}-H_{t}, \quad \sigma_{\eta}^{2}-1 \text { e } \quad \sigma_{z}^{2}=1 / 4
$$

para $l=1,2, \ldots$, ficanos com um modelo linear invariante no tempo da forma

$$
\begin{aligned}
& y_{t}=a \nu_{l}+b+\varepsilon_{l} \quad \text { (observacano) } \\
& \nu_{l}=\left(\eta_{t}+1+r \eta \eta \quad\right. \text { (cstaklo). }
\end{aligned}
$$

E tambenu, E, e $\eta_{t}$ são independentes para todo $b$, e independentes do valou inicial $\nu_{0}$. Assumimos que?

$$
\nu_{0} \sim \mathcal{N}\left(\bar{\nu}_{0}, \Gamma_{0}\right), \quad \bar{\nu}_{0} \text { e } P_{0} \text { conlecirlos. }
$$

Como feito na obtençäo rlo FK, o estado para $t=1$ fica descrito como

$$
\nu_{1}=c \nu_{0}+d \mid r \eta_{1}
$$

Dirctamento conchún nos gue:

$$
\nu_{1} \mid \nu_{0} \sim \mathcal{N}\left(\bar{\nu}_{1 \mid 0,}, \Gamma_{10}^{\prime}\right)
$$

oll give

$$
\bar{\nu}_{1 j 0}=c \bar{\nu}_{0}+d
$$

$$
l_{1,10}^{\prime}=r^{2} l_{0}^{\prime}+r^{2} \sigma_{\eta}^{2}
$$

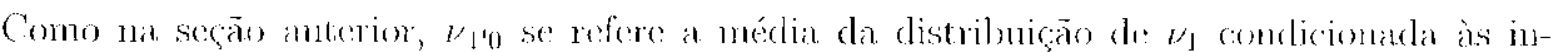
Comações até $1=0$. Escrevendo

$$
\begin{gathered}
\nu_{1}=\bar{\nu}_{1,0}+\left(\nu_{1}-\bar{\nu}_{1 ! 0}\right) \\
y_{1}=a \bar{\nu}_{1,0} \mid a\left(\nu_{1}-\bar{\nu}_{1 \mid 0}\right)+b+\varepsilon_{1}
\end{gathered}
$$

conchumos que o vetor $\left(\nu_{1} \quad y_{1}\right)$ tem distribugăo conjunta nomal bivariada, com vetor de medias $\left(\bar{\nu}_{3,0} \quad a \bar{\nu}_{10}+b\right)$ e matriz de variancias

$$
\left(\begin{array}{cc}
I_{1 ! 0} & a P_{1 ! 0} \\
a P_{1 \mid 0} & a^{2} P_{1 ! n}+\sigma_{s}^{2}
\end{array}\right)
$$


Do lema 3.1 segue que

$$
\nu_{1} \|_{1} \sim \mathcal{N}\left(\bar{l}_{1}, \Gamma_{1}\right)
$$

?His (100)

$$
\begin{gathered}
\ddot{\nu}_{1}=\bar{\nu}_{1 ; 0}+a P_{1 ! 0} f_{1}\left(y_{1}-a \bar{\nu}_{1 \mid 0}-b\right) \\
P_{1}-P_{1 \mid 0}-a^{2} P_{1 \mid 0}^{2} f_{1}^{\cdots-1}
\end{gathered}
$$

$$
f_{i}=a^{2} P_{i 0}+\sigma_{i}^{2}
$$

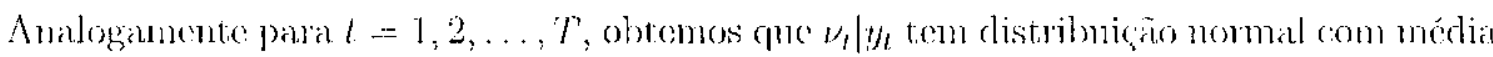

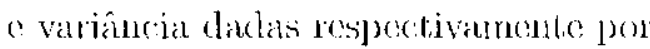

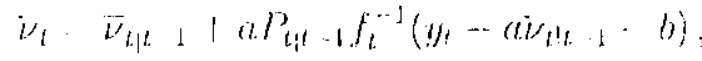

$$
\begin{aligned}
& P_{i} \because P_{i \mid t-1-1}-a^{2} P_{i l i-1}^{3} I_{i}{ }^{\prime}
\end{aligned}
$$

$$
J_{l}=a^{2} r_{t \mid l \cdot 1}+o_{c}^{2}
$$

on que $\bar{\nu}_{1+1 \mid t}$ e $P_{t-1 \mid t}$ säo obtidos como nas cquacous (3.8) e (3.9), ou seja.

$$
\bar{\nu}_{t, l-1}=\left(\bar{p}_{t-1}+d\right.
$$

$$
P_{i j \cdot 1}=r_{1}^{2} H_{1}+r^{2} \sigma_{1}^{2}
$$

\subsubsection{Verossimilhança}

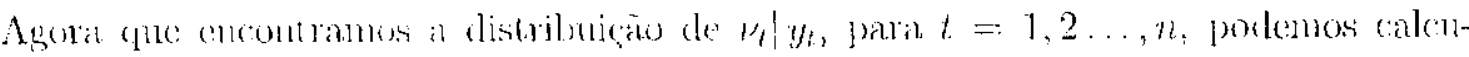

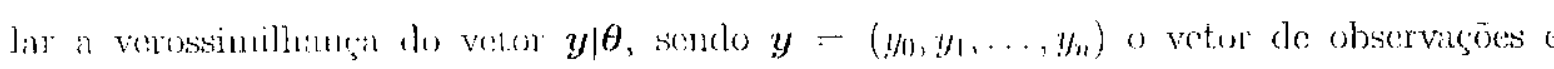

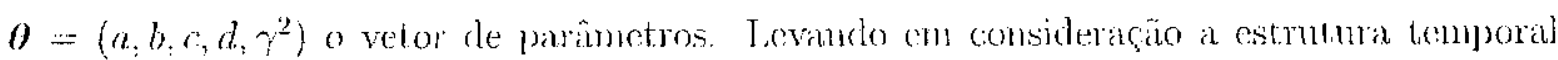

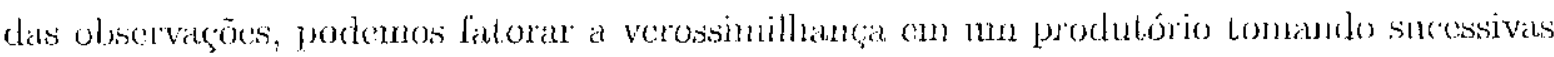
condicionais

$$
L(\theta)=p(\boldsymbol{y} \mid \boldsymbol{\theta})=\mu\left(y_{0} \mid \boldsymbol{\theta}\right) \prod_{i=1}^{n} \mu\left(y_{i} \mid \boldsymbol{y}_{-1}, 0\right)
$$

an que $y_{t-1}-\left(y_{0}, y_{1}, \ldots, y_{t-1}\right)$, on seja, denota o comjunto de informatçöes obticlas até o instinte : - 1 . 
Pela cquaras (3.35) hemos que

$$
y_{0}-\left(1 l_{0}+b+c\right)
$$

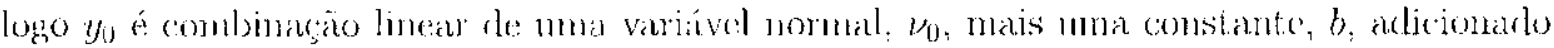

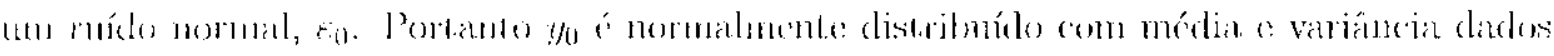
res]ectivamente por

$$
\bar{y}_{0}=a \bar{y}_{0}+b
$$

$$
\sigma_{0}^{2}=a^{2} P_{0}^{2}+\sigma^{2}
$$

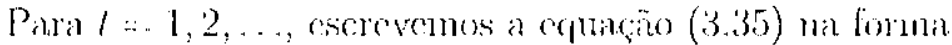

$$
y_{1}=a \bar{x}_{1 ! 1}+a\left(x_{1}-\bar{x}_{1,1}\right)+b+\bar{z}_{1} .
$$

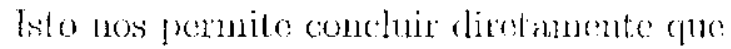

$$
y_{l} \mid y_{l,}, \sim \mathcal{N}\left(y_{t}, \sigma_{i}^{2}\right)
$$

('om)

$$
\bar{y}_{1}=\left(a \bar{w}_{1+\cdots}+b\right.
$$

$$
\sigma_{l}^{2} \cdots j_{l}
$$

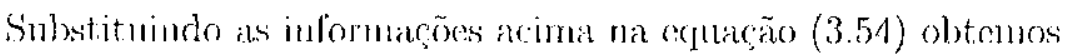

$$
l(\theta)=\nu(y ! \theta) \propto \prod_{i=1}^{n}\left(\frac{1}{\sigma_{i}^{2}}\right)^{1 / 2} \exp \left\{-\frac{\left(y_{t}-\bar{y}_{l}\right)^{2}}{2 \sigma_{t}^{2}}\right\} .
$$

Devemos maximiza a expario (3.62) on, equivalentemente, maximizar sen logarituo, a log-verosisimithranga

$$
l(\theta) \therefore \ln l,(\theta) \propto-\frac{1}{2} \sum_{l, 0}^{n} \ln \left(\sigma_{l}^{2}\right)-\frac{1}{2} \sum_{l-0}^{n} \frac{\left(y_{l}-\bar{y}_{l}\right)^{2}}{o_{l}^{2}}
$$

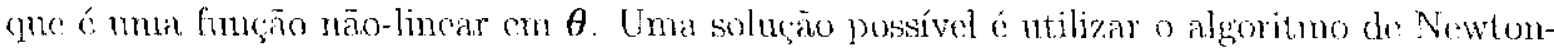

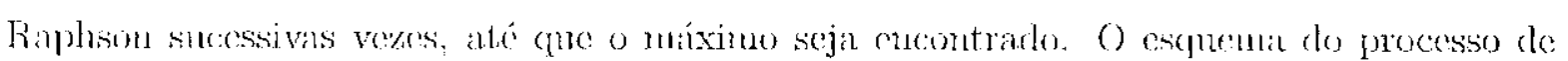

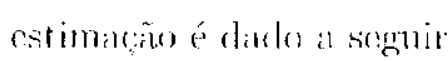


1. inicia com valores $\theta^{(i)}$ e contador de iteração $j=0$;

2. utiliza o FK o o vetor inicial; $\theta^{(j)}$, para obter o conjunto de esperamcats e varianciats, $\left\{\bar{y}_{i}^{(j)}: \sigma_{i}^{2(j)}\right\}$

3. executa uma iteraçäo ro algoritno de Aewton-Raphson ntilizando os valores obtidos 110 passo 2 e obtendo un novo conjunto de cslinativas, $\theta^{(j+1)}$.

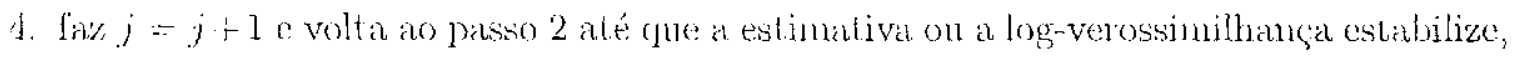
on sejach, até rane

$$
\left\|\theta^{(j+1)}-\theta^{(j)}\right\|<\xi \quad \text { ou } \quad\left|l\left(\theta^{(j+1)}\right)-l\left(\theta^{(j)}\right)\right|<\xi,
$$

parac $\xi>0$ cliaclo

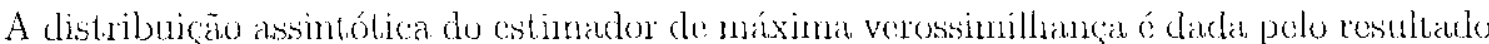
cominciado al segruir.

Teorema 3.2. Sob condiçoes gerais, sejac $\widehat{\theta}_{N}$ o extinador de máxinna verossinnilisança de $\theta$, obtido maximizando a expressão (3.63). Futtĩu, gutando $N \rightarrow \infty$,

$$
\sqrt{N}\left(\hat{\boldsymbol{\theta}}_{N}-\boldsymbol{\theta}\right) \stackrel{D}{\rightarrow} \mathcal{N}\left(0, I(\theta)^{-1}\right)
$$

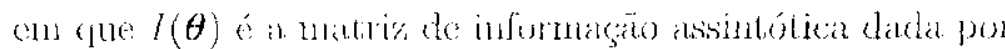

$$
I(\theta)=\lim _{N \rightarrow \infty} N^{\prime} E\left[-\frac{\partial^{2} l(\theta)}{\partial \theta} \dot{\partial \theta} \theta^{\prime}\right]
$$

Prova. (Veja p.ex. Caines, 1988).

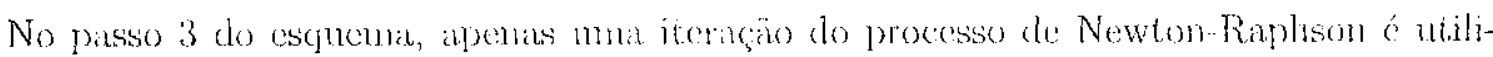

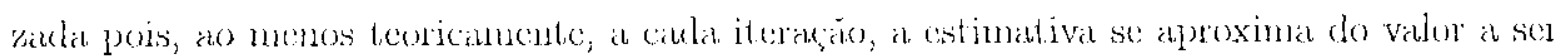

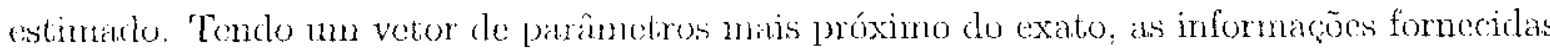
pelo FK deven ser atmalizadas. Feita a atnalizayäro, novamente buscamos a proximidade do valor exato.

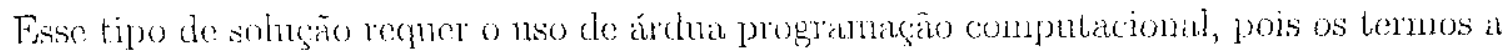

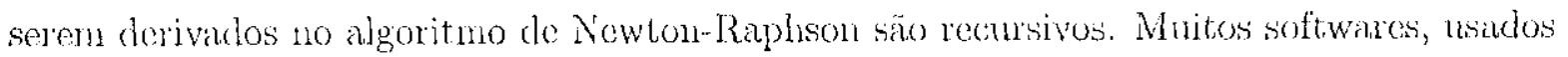


primeimamente na área de engenlaura, propöen-se a solncionar mumericanente o problema dar diferenaciano.

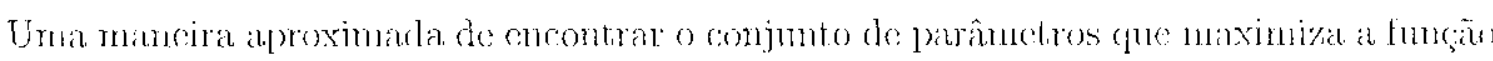

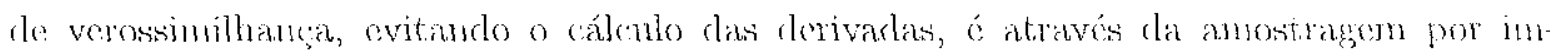

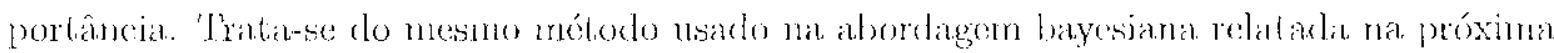
sulseçäo. A icléia é construir una amostra cle estimativas clos parametros que represento

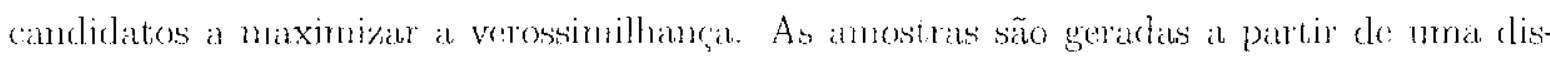

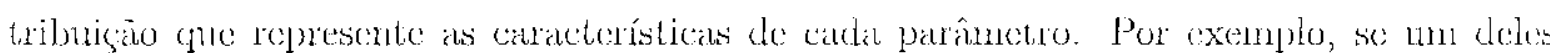

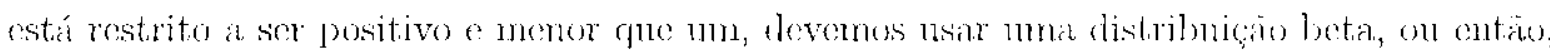

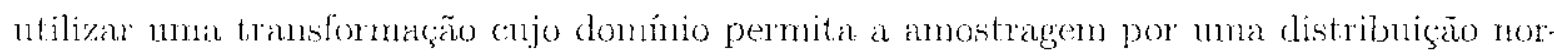

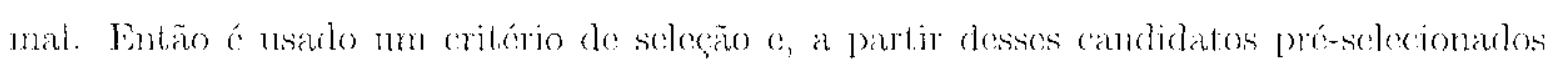
estimamos os partinetros.

\subsubsection{Abordagem Bayesiana}

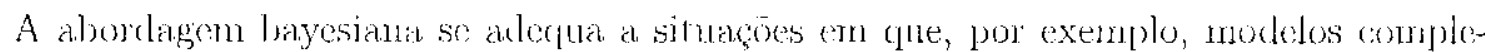

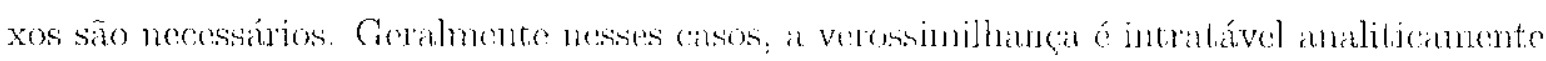
Outro exemplo, é a possibilidade de inserir combecinentos próvios a respeito ra comporta

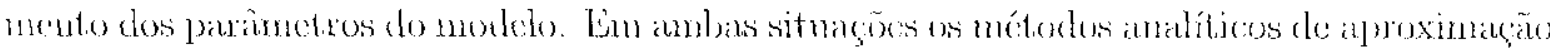

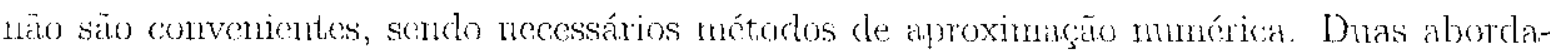

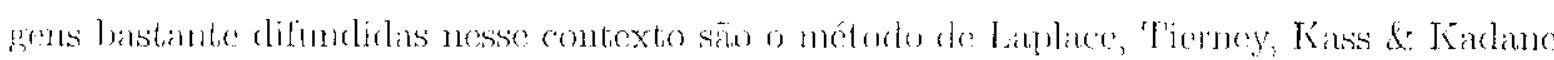

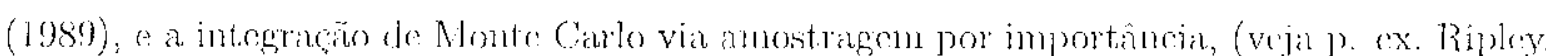

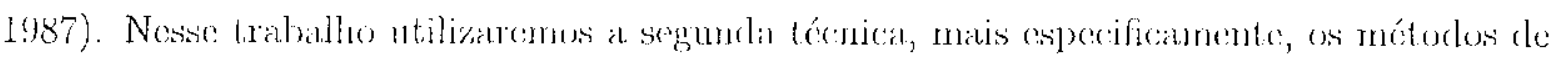
Monte Carlo via carleias de Markov (MCMC). É um método de simples implennentagão e

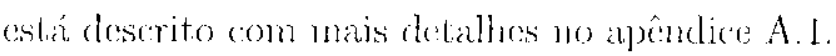

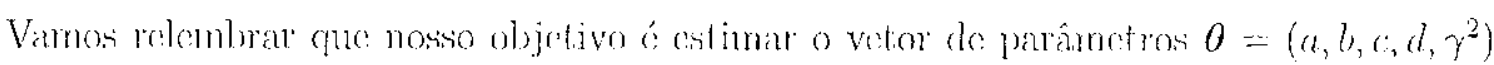

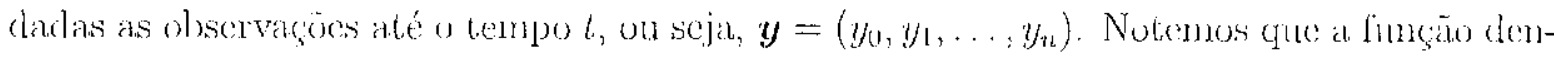

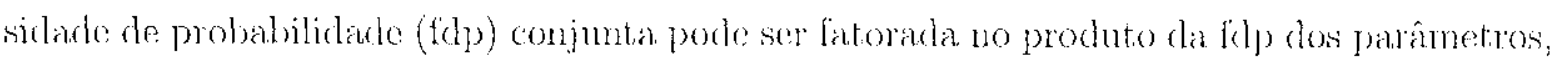

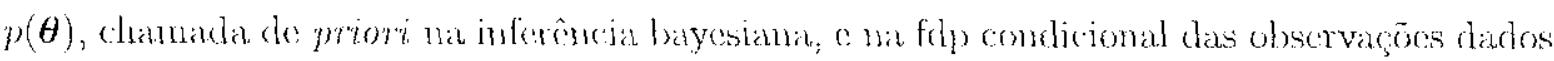

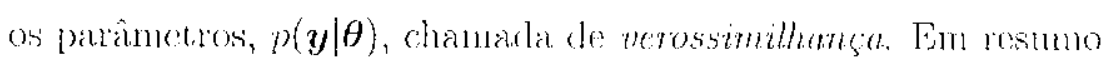

$$
p(\boldsymbol{\theta}, \boldsymbol{y})=p(\boldsymbol{y} \mid \theta) p(\boldsymbol{\theta}) .
$$


Depois de coletar os dados, alualizamos o conhecimento a priori polo teorema de Bayes, o que rosulta na posterioni, $p(\theta \mid y)$,

$$
\mu(\boldsymbol{\theta} \mid \boldsymbol{y})=\frac{p(\boldsymbol{y} \mid \boldsymbol{\theta}) p(\boldsymbol{\theta})}{\nu(\boldsymbol{y})}
$$

em que $p(\boldsymbol{y})=\int \mu(\boldsymbol{y} \mid 0) p(\boldsymbol{\theta}) d \theta$ é a lidp marginal ale $\boldsymbol{y}$

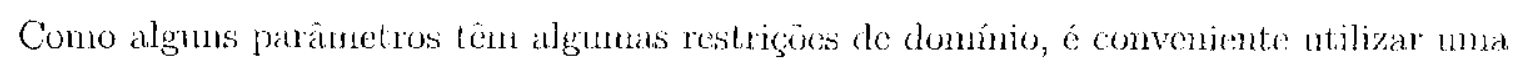
transfornação destes no processo de estimação. O parânuetro e está restrito a assumir valoress entre 0 e 1, ja $\gamma^{2 *}$ a assumir valores positivos, por isso traballatiemos con

$$
c^{*}=\log \frac{c}{i-c}, \quad \text { on scja, } \quad c-\frac{\exp \left(c^{*}\right)}{1+\exp \left(r^{*}\right)}
$$

e. cont

$$
\gamma^{2 *}=\exp \left(\gamma^{2}\right), \quad \text { ou seja } \quad \gamma^{2}=\log \left(\gamma^{3 *}\right)
$$

Considerenos que os parânetros săo independentes e que tenlam fọp a priori nomais tajs (1110

$$
\begin{aligned}
& a \cdots \mathcal{N}\left(\mu, \sigma_{a}^{2}\right), \quad b \sim \mathcal{N}\left(\mu, \sigma_{b}^{2}\right) \\
& c^{*} \sim \mathcal{N}\left(\mu_{c^{*}}, \sigma_{c^{+}}^{2}\right), \quad d \sim \mathcal{N}\left(\mu_{l}, \sigma_{d}^{2}\right) \quad\left(\quad \gamma^{2 *} \sim \mathcal{N}\left(\mu_{\gamma^{2 *}}, \sigma_{\gamma^{2 *}}^{2}\right)\right.
\end{aligned}
$$

Entăo

$$
\begin{aligned}
p(\theta) & =p(a) p(b) p\left(c^{*}\right) p(d) p\left(\gamma^{2 *}\right) \\
& =\prod_{r=a, b_{r} c^{*} t \gamma^{2 *}} \frac{1}{\sqrt{2 \pi} \sigma_{r}} \exp \left\{\frac{1}{2 \sigma_{r}^{2}}\left(r-\mu_{r}\right)^{2}\right\} \\
& =\left(\frac{1}{2 \pi}\right)^{5 / 2} \frac{1}{\sigma_{u} \sigma_{b} \sigma_{v^{*}} \sigma_{d} \sigma_{\gamma^{2 *}}} \exp \left\{-\sum_{r} \frac{1}{2 \sigma_{r}^{2}}\left(r-\mu_{r}\right)^{2}\right\} .
\end{aligned}
$$

Multiplicando as equaçoes (3.62) e (3.73) obtemos que a posteriori é proporcional a

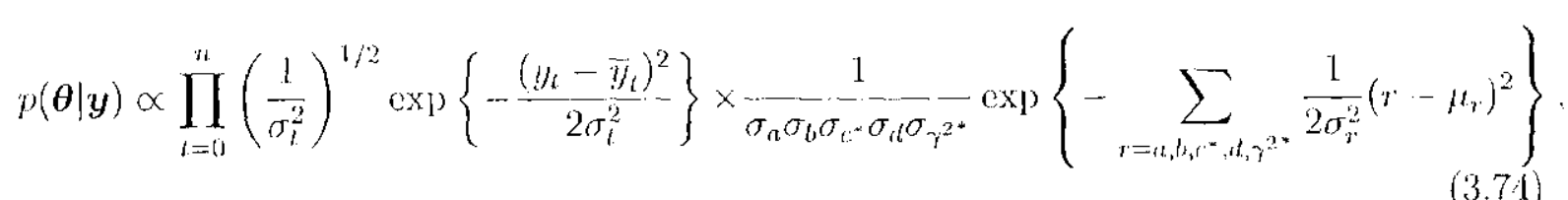

a mesma expressäo porle ser escrila cono

$$
p(\boldsymbol{\theta} \mid \boldsymbol{y}) \propto \exp )\left\{-\sum_{t=0}^{n} \frac{\left(y_{t}-\bar{y}_{t}\right)^{2}}{2 \sigma_{t}^{2}}-\sum_{r=a, b, c^{*}, d_{,} \gamma^{2}} \frac{\left(r-\mu_{r}\right)^{2}}{2 \sigma_{r}^{2}}\right\} \prod_{t=0}^{n}\left(\frac{1}{\sigma_{t}^{2}}\right)^{1 / 2}
$$

já r pue $\bar{y}_{l}$ e $\sigma_{l}^{2}, l=0,1, \ldots, n$, dependem do vetor de parânetros $\theta$. 


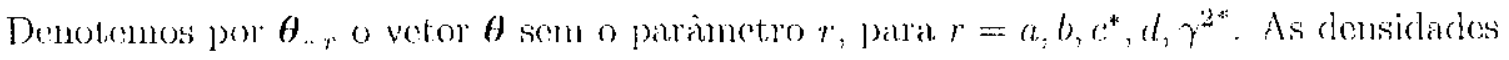
conticionalis a posteriori säo proporcionats a.

$$
\left.\left.p\left(r \mid y, \theta_{\ldots r}\right) \propto \operatorname{ex}\right]\right)\left\{-\sum_{t=0}^{n} \frac{\left(y_{t}-y_{t}\right)^{2}}{2 \sigma_{t}^{2}} \ldots \frac{\left(r \cdot \mu_{r}\right)^{2}}{2 \sigma_{r}^{2}}\right\} \prod_{t:-0}^{n}\left(\frac{1}{\sigma_{t}^{2}}\right)^{1 / 2}, \quad r=a, b, r^{*}, d, \gamma^{2+}
$$

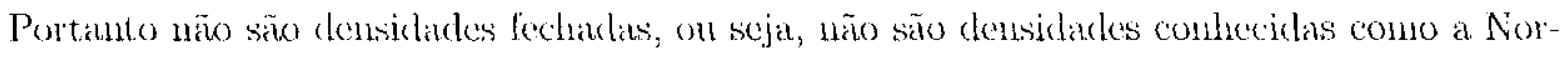

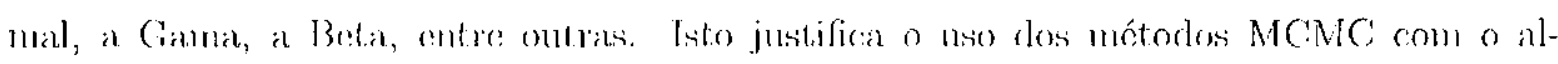
goritmo de Metuopolis-Fastings resenvolvido por Metropolis, Rosenbluth, Teller d te Tello

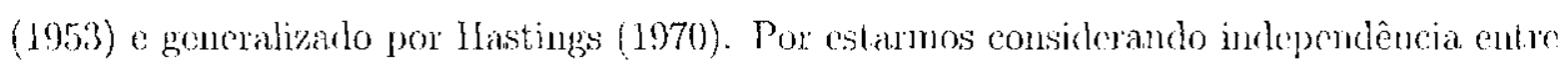

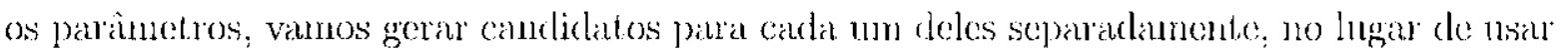

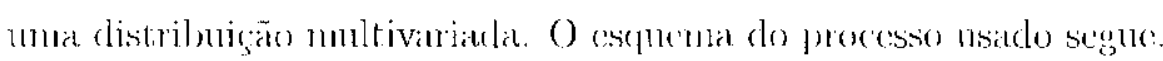

1. inicta com valon $\theta^{(0)}$ e combator de itoração $j=0$;

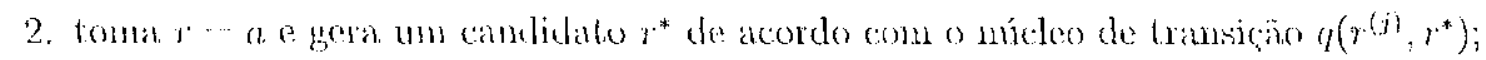

3. atualiza $r^{(0)}$ por $r^{(j \mid 1)}-r^{*}$ com probabilidade

$$
p=\min \left\{1, \frac{p\left(r^{*}\right) q\left(r^{(j)}, r^{*}\right)}{p\left(r^{(o)}\right) q\left(r^{*}, r^{(j)}\right)}\right\}
$$

ou seja, permanee com $r^{(j)}$ com probabilidarle $1-p$;

1. toma $r=6$ o repete os passos 2 e 3 ;

5. loma $t-c^{+}$a repete os passos 2 e 3 ;

6. tonta $1-$ d c repete os passos 2 e 3 ;

7. tomar $r \cdot \gamma^{2 *}$ erepete os patssos 2 a 3 ;

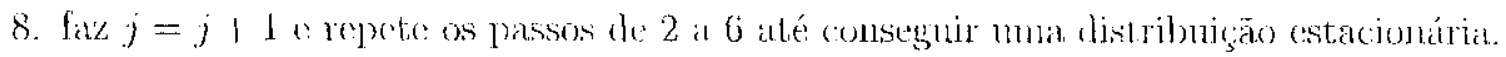

Bsste processo nos fornece nun conjunto de estimativas usadas na inferencia dos paranmetros. $\Lambda$ lgumas aplicacoes podem ser vistas no capítulo 5 . 


\subsection{Conclusão}

Neste capínlo apresentamos a construçăo do FK, assirn como a fumçăo de verossimilhança de un processo de estado. 'Tiatanos do num modelo invariante no tempo, apresen-

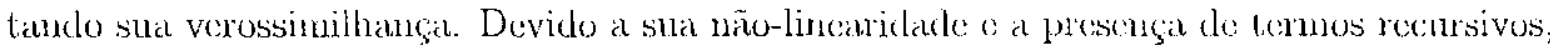

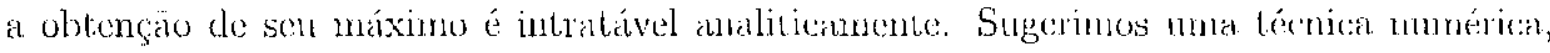

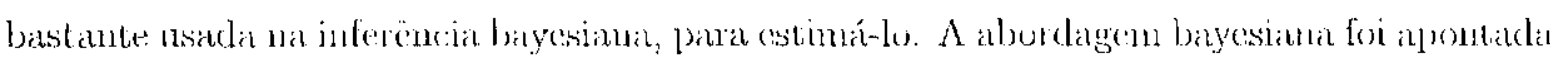

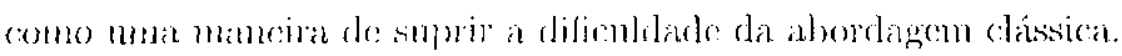




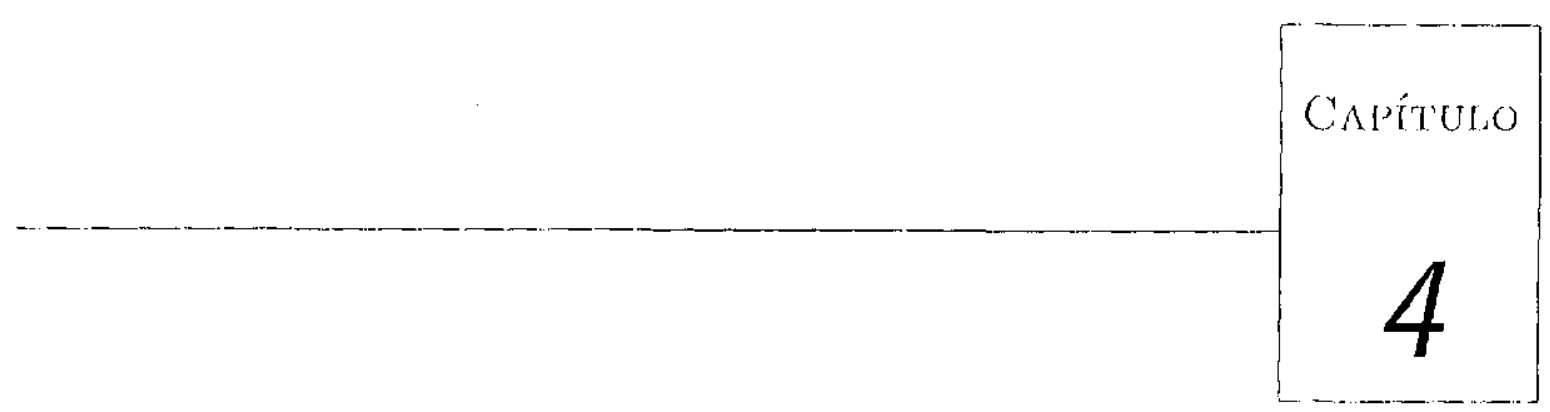

Inferência para o Modelo I

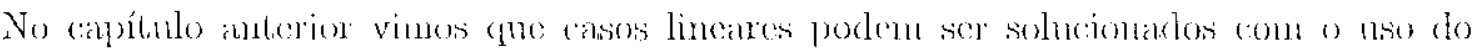

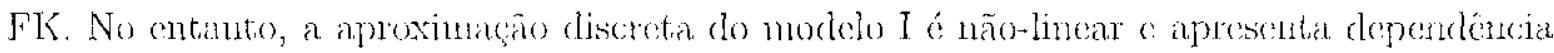
do estato na variancia. Para a inferencia deste tipo de modelo sugerinos o uso do filtro

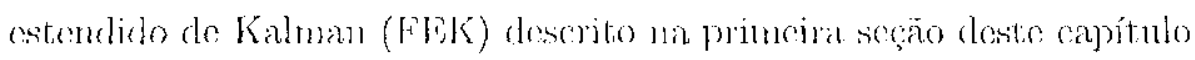

\subsection{Filtro Estendido de Kalman}

Considere oseguinte modelo näo-linear em estaklo de espaço

$$
\begin{aligned}
& y_{t}=A_{t}\left(t_{t}\right)+\varepsilon_{t} \quad \text { (observicio) }
\end{aligned}
$$

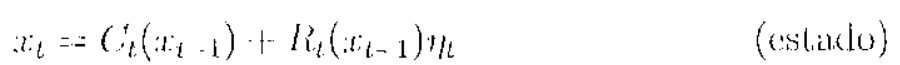

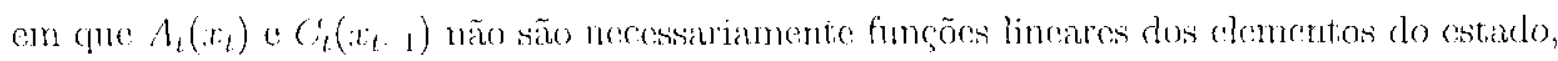

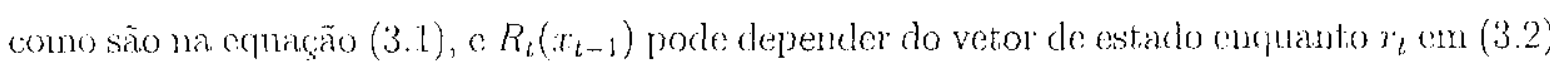

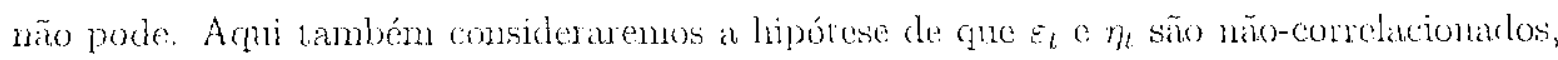

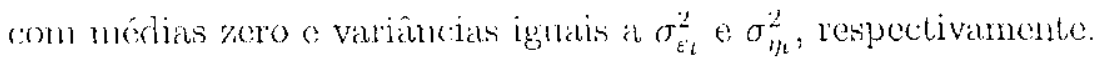




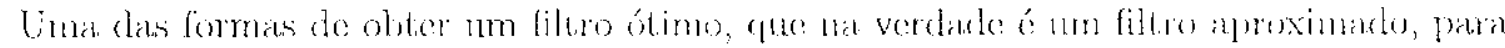

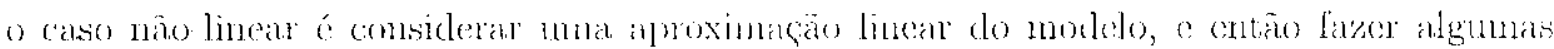

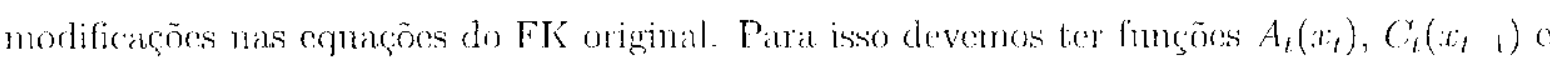
$R_{t}\left(x_{i}, 1\right)$ sulfejentemente suaves, tais que sejann possiveis de serenn expandiclas em séries de Taylor enn torno de suns médias condicionais e médias, $\bar{x}_{t \mid t}+$ e $\bar{x}_{t, \cdots}$

$$
\begin{aligned}
& A_{t}\left(x_{t}\right) \simeq A_{t}\left(x_{t \mid l-1}\right)+\frac{\partial A_{t}}{\partial y_{l}}\left(\bar{x}_{t \mid l-1}\right)\left(x_{t}-\bar{x}_{t \mid l-1}\right), \\
& C_{t}\left(x_{t-1}\right) \simeq C_{l}\left(\bar{x}_{t-1}\right)+\frac{\partial C_{t}^{r}}{\partial x_{t}}\left(\bar{x}_{t-1}\right)\left(x_{t-1}-\bar{x}_{t-1}\right)
\end{aligned}
$$

$$
R_{l}\left(x_{t-1}\right) \simeq R_{l}\left(\bar{x}_{t-1}\right)
$$

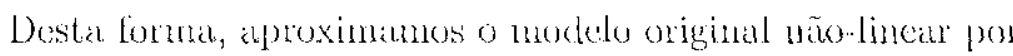

$$
\begin{aligned}
& y_{t} \simeq \frac{\partial A_{t}}{\partial x_{t}}\left(\bar{x}_{t \mid t-1}\right) x_{t}+A_{t}\left(\bar{x}_{t \mid+1}\right)-\frac{\partial A_{t}}{\partial x_{t}}\left(x_{t \mid t-1}\right) \bar{x}_{t \mid t-1}+1 \cdot \varepsilon_{t} \\
& x_{t} \simeq \frac{\partial C_{t}}{\partial x_{t}}\left(x_{t}\right) x_{t}+C_{t}\left(\bar{x}_{t-1}\right)-\frac{\partial C_{t}}{\partial x_{t}}\left(x_{t-1}\right) \bar{x}_{t}+R_{t}\left(\bar{x}_{t-1}\right) r_{t},
\end{aligned}
$$

que ainda pode ser resscrito considerando

$$
\begin{aligned}
& a_{l}=\frac{\partial A_{t}}{\partial a_{l}}\left(a_{t h-1}\right), \\
& c_{l}=\frac{\partial C_{l}}{\partial r_{l}}\left(\bar{r}_{t} 1\right)
\end{aligned}
$$

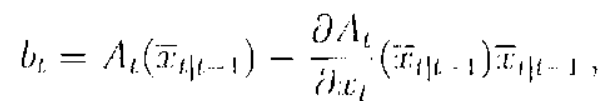

$$
\begin{aligned}
& d_{l}=C_{l}\left(\bar{x}_{t-1}\right)-\frac{\partial C_{t}}{\partial x_{t}}\left(\bar{x}_{t},\right) \bar{x}_{t-1}
\end{aligned}
$$

$$
r_{t}-h\left(\bar{x}_{t-1}\right)
$$

Hat forma

$$
\begin{aligned}
& y_{l} \simeq a_{l} x_{l}+b_{t}+\varepsilon_{i} \\
& r_{t} \simeq a_{l} x_{l-1}+d_{l}+r_{l} \eta_{l}
\end{aligned}
$$

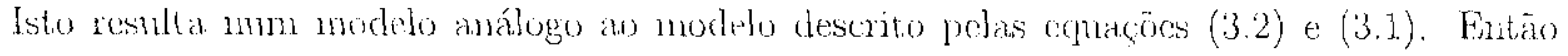
aplicanos o FK ao modelo aproximarlo, obtendo conno expacões de predicão

$$
\bar{x}_{t \mid t-1}=C_{i}\left(\bar{x}_{t, 1}\right)
$$




$$
P_{t \mid t-1}=c_{l}^{2} P_{t-1}+r_{t}^{2} \sigma_{\eta_{t}}^{2}
$$

e como equações de athalizaz̧ão

$$
\begin{gathered}
\bar{x}_{t}=\bar{x}_{l \mid t-1}+a_{t} \rho_{t \mid t-1} f_{t}^{-1}\left(y_{t}-A_{t}\left(\bar{x}_{t \mid t-1}\right)\right), \\
P_{t}=P_{t \mid t-1}-a_{t}^{2} P_{t \mid t-1}^{2} f_{t}^{-1}
\end{gathered}
$$

$$
f_{t}=a_{t}^{2} P_{t \mid t-1}+\sigma_{\varepsilon_{t}}^{2}
$$

Em sintese, aplicamos o FK a uma aproximação linear do modelo original, dada pelas ecuações (4.13) e (4.14), e obtemos o mesmo filtro descrito no capítulo anterior utilizando, no lugar das cquações (3.26) o (3.28), as equações (4.15) e (4.17). Essas duas últimas cquações servem apenas para evitar a soma e subtração de termos que se cancelarn quando utilizanos as equações do FK obtidas no capítulo anterior.

O objetivo do FEK é análogo ao objetivo do FK, ou seja, ó estimar a distribuição do estarlo no instante t condicionado às observacöes obtidas até $t$. Nesse caso ela é aproxintada por uma nornıal com média (4.17) e variância (4.18), a qual denotaremos por $\tilde{p}\left(x_{l} \mid \boldsymbol{y}_{t}, \boldsymbol{\theta}\right)$.

Observação 4.1. Em geral não há uma trajetótia de referência en tono da qual o sistema evolui. O FK adota como trajetória de referência os valores estimados. Estie é o motivo de tomar as expansões en Taylor em torno das médias condicionais.

Como agora estannos tratando de um caso lincar, mesmo sendo este uma aproximação a um outro, o procedimento para a obtenç̃̃o da verossimilhança é análogo ao do capítulo anterior: Se o processo é invariante no tempo, sua verossimilhança pode ser descrita pela equação (3.62), considerando as mudanças nas equações de predição e de atualização dadas acima.

\subsection{Modelos Condicionalmente Gaussianos}

Aqui apenas mostramos que os modelos podem ser um pouco mais abrangentes. Muitas vezes os coeficientes do processo não dependem somente do tempo, mas também das observaçōes obtidas até o instante anterior, $t-1$. Condicionando o modelo ao conjunto de 
informaçöes obticlas até $t-1$, ficamos com um modelo condicionalmete gaussiano. Fsse pode ser escrito explicitanente por

$$
\begin{array}{ll}
y_{t}=a_{l}\left(\boldsymbol{Y}_{t-1}\right) x_{t}+b_{t}\left(\boldsymbol{Y}_{t-1}\right)+\varepsilon_{t} & \text { (observagão) } \\
x_{l}=c_{l}\left(\boldsymbol{Y}_{t-1}\right) x_{t-1}+d_{l}\left(\boldsymbol{Y}_{t-1}\right)+r_{t}\left(\boldsymbol{Y}_{t-1}\right) \eta_{l} & \text { (estado) }
\end{array}
$$

em que $\varepsilon_{t}\left|\boldsymbol{Y}_{t-1} \sim \mathcal{N}\left(0, \sigma_{\varepsilon_{t}}\left(\boldsymbol{Y}_{t-1}\right)\right), \eta_{t}\right| \boldsymbol{Y}_{t-1} \sim \mathcal{N}\left(0, \sigma_{\eta_{t}}\left(\boldsymbol{Y}_{t-1}\right)\right)$ e $x_{0} \sim \mathcal{N}\left(\bar{x}_{0}, P_{0}\right)$. O sistema dewe ser considerado fixo, já que estamos no tempo t. Por isso, as cquarónes do FK e as do FEK são obtidas como anteriormente.

\subsection{MVEM}

Lembremos que os MVEN tratados neste trabalho têm equaŗão de obscrvação dada por

$$
H_{t+h}=H_{t}+h\left(\mu-\frac{\sigma_{l}^{2}}{2}\right)+h^{1 / 2} \sigma_{t} Z_{t+h} .
$$

Notemos que o ruído presente na equação das observações está multiplicado por uma função dos cobtados. Isto impossibilita a aplicarão completa do FEK, já que não temos mais a condiçäo de nomalidade do ruído satisfeita. Vale ressaltar que, mesmo condicionando esse modelo a conjunto de observaçōes passadas, a hipótese de normalidade do erro ó corrompida.

Usaremos então apenas o primeiro estágio do FEK. Esse estágio nos fornece a fdp da valuável latente no tempo $l$ condicionada ao conjunto de informacones até $l-1$. Como já foi dito anteriomente, o inconveniente disso é que apenas podemos csitimar os parânetros do modelo, näo tendo un filtro capaz de estimar o estarlo. Na próxima seção obteremos a verossimilhança de um processo geral, que atende às condições dos MVEM

\subsubsection{Verossimilhança}

Obter a verossimilhança condicionada às variáveis de estado de um MVEM não é tão simples como 110 caso linear on conno no caso aproximalamente linear. Nos MVEM a verossimilhança não é dada por uma forma fechada e requer uma integracão n-dimensional sobre o vetor cle cstados $\boldsymbol{x}=\left(x_{1}, x_{2}, \ldots, x_{n}\right)$ como vemos

$$
L(\boldsymbol{\theta})=\int p(\boldsymbol{y}, \boldsymbol{x} \mid \boldsymbol{\theta}) d \boldsymbol{x}=\int p(\boldsymbol{y} \mid \boldsymbol{x}, \boldsymbol{\theta}) p(\boldsymbol{x} \mid \boldsymbol{\theta}) d \boldsymbol{x}
$$


Levando em consicleaçĩo a estnutura temporal das observaçöes latoramos a verossimilhança através de sucessivas condicionais obtenclo

$$
L(\boldsymbol{\theta})=p\left(y_{0} \mid \boldsymbol{\theta}\right) \prod_{t=1}^{n} p\left(y_{t} \mid \boldsymbol{y}_{t-1}, \boldsymbol{\theta}\right)
$$

em que $\boldsymbol{y}_{t-1}=\left(y_{0}, y_{1}, \ldots, y_{t-1}\right)$ é o vetor de observaçóes coletarlas até o tempo $t-1$. Carla fator do produtónio acima é dado pelas integrais

$$
p\left(y_{0} \mid \theta\right)=\int p\left(y_{0} \mid x_{0}, \theta\right) p\left(x_{0} \mid \theta\right) d x_{0}
$$

$\therefore$ parat $t=1,2, \ldots, n$,

$$
p\left(y_{l} \mid \boldsymbol{y}_{l-1}, \boldsymbol{\theta}\right)=\int p\left(y_{t} \mid x_{t}, \boldsymbol{\theta}\right) p\left(x_{t} \mid \boldsymbol{y}_{t-1}, \boldsymbol{\theta}\right) d x_{t_{t}}
$$

Isto reduz a integral n-dimensional en (4.23) a 1 m produto de $n$ integrais unidimentonatis

Analiscmos a integral on (4.25). Conno yo não é combinaçăo lincar de $x_{0}$, tal intogral não fica fácil de ser obtida. O traballso de Meyer, Fommer \& Berg (2003) usou a aproximagäro

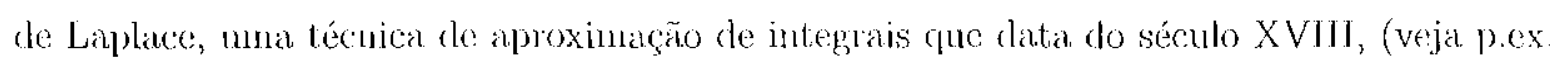
Laplace, 1986) e veja apêndice A.2. Através dessa técnica temos

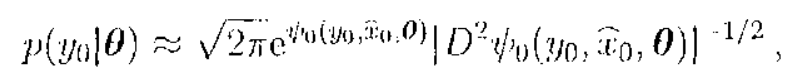

en que

$$
\psi_{0}\left(y_{0}, x_{0}, \boldsymbol{\theta}\right)=-\log \left(p\left(y_{0} \mid x_{0}, \boldsymbol{\theta}\right) p\left(x_{0} \mid \theta\right)\right)
$$

$\widehat{x}_{0}$ é o múnino da função tho con relação a $x_{0}$, e $D^{2} \psi_{0}\left(y_{0}, x_{0}, \theta\right)$ denota a derivada de segunda orden da lunçĩo $\psi_{0}\left(y_{0}, x_{0}, \theta\right)$ com relaçió a $x_{0}$.

Tondo apenas a observaju inicial, podemos, através do teorema de Bayes, atualizar a densidade $p\left(x_{0} \mid \theta\right)$ do estado desconhecido pela densidade de filtragem $p\left(x_{0} \mid y_{0}, \theta\right)$ como mostra a equaço

$$
p\left(x_{0} \mid y_{0}, \theta\right)=\frac{p\left(y_{0} \mid x_{0}, \theta\right) p\left(x_{0} \mid \theta\right)}{p\left(y_{0} \mid \theta\right)} .
$$

Sna distribuição pode ser aproximada por una distribuiçăo nomal dada por

$$
\mathcal{N}\left(\widehat{x}_{0}, I^{-1}\left(\widehat{x}_{0}\right)\right),
$$

em que

$$
I\left(\widehat{x}_{0}\right)=-\left.\frac{\partial^{2}}{\partial x_{0}^{2}} \log \left(p\left(y_{0} \mid x_{0}, \theta\right) p\left(x_{0} \mid \theta\right)\right)\right|_{x_{0}-\widehat{x}_{0}},
$$


on seja, ignal a informação de Fisher olscervada da funçäo log $\left(p\left(y_{0} \mid x_{0}, \theta\right) p\left(x_{0} \mid \theta\right)\right.$, (veja pex. Sen \& Singer; 1993). Aincla vale nolats gue

$$
I\left(\widehat{x}_{0}\right) \cdot \quad D^{2} \psi_{0}\left(y_{0}, \widehat{x}_{0}, \theta\right)
$$

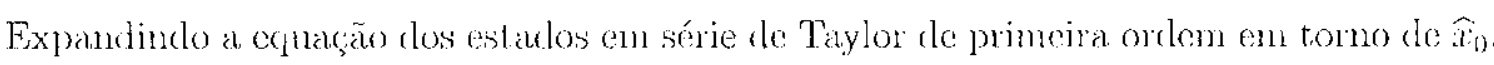

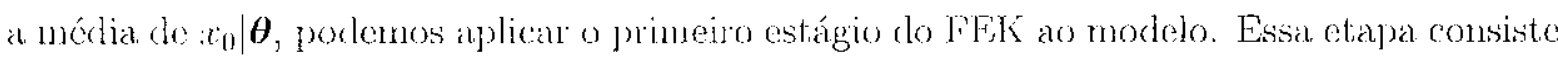
em estima a média e a variancia do estado no próximo instante de tempo, condicionarlo ao conjunto rle informaçoes obtiulas até então, no caso, $x_{3} \mid y_{0}, \theta$. Note que nessa abordagenu, a variância da filtragem, denotada anterionnente por $P_{0}$, c dada por $I^{-1}\left(\widehat{x}_{0}\right)$, e sua média, ao invés rle ser o valor estinado do estado, é dada por $\widehat{x}_{0}$. Éntã̃o, pelas equacōes (1.15) e (4.16)

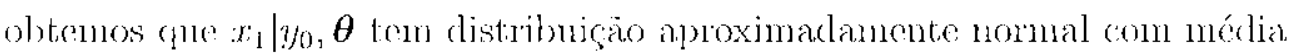

$$
\bar{x}_{1 \mid 0}=C_{1}\left(\widehat{x}_{0}\right)
$$

e variância

$$
I_{\beta \mid 1}-c_{1}^{2}\left(\hat{x}_{0}\right) I^{-1}\left(\widehat{x}_{0}\right)+r_{1} \sigma_{\eta}^{2} .
$$

Analogamente para $t=1,2, \ldots, n$, a felp preditiva de $x_{t} \mid y_{t-1}, \theta$ é aproximakla por uma normal, $\tilde{p}\left(x_{1} \mid \boldsymbol{y}_{l}, \boldsymbol{\theta}\right)$, com unćdia e variância daclass, respectivanente, por

$$
x_{i \mid 1-1}=C_{1}\left(\hat{x}_{1-1}\right)
$$

e

$$
P_{t \mid t-1}=\left\lfloor c_{t}\left(\hat{x}_{t-1}\right)\right\rfloor^{2} I^{-1}\left(\widehat{x}_{t-1}\right)+r_{t}^{2} \sigma_{\eta}^{2}
$$

Jombrame que, repois de olsservar $y_{t}$, a felp $p\left(x_{t} \mid y_{l}, \theta\right)$ é atualizala através do teoroma de Bayes por

$$
p\left(x_{t} \mid y_{t}, \theta\right) \propto \eta\left(y_{h} \mid x_{t}, \theta\right) p\left(x_{t} \mid y_{l-1}, \theta\right) \approx \eta\left(y_{t} \mid x_{t}, \theta\right) \tilde{p}\left(x_{l} \mid y_{t, 1}, \theta\right)
$$

inplicando que $x_{\imath} \mid y_{\ell}, \theta$ tem felp $\eta\left(x_{l} \mid y_{l}, \theta\right)$ aproximadamente normal com mérlia $\widehat{x}_{t}$ e variância $\gamma^{-1}\left(\widehat{x}_{1}\right)$.

Findmente poclemos aproximar os termos on (1.26) através de

$$
p\left(y_{l} \mid y_{t-1}, \theta\right) \approx \int p\left(y_{l} \mid x_{t}, \theta\right) \widetilde{p}\left(x_{i} \mid y_{t-1}, \theta\right) d x_{t}
$$

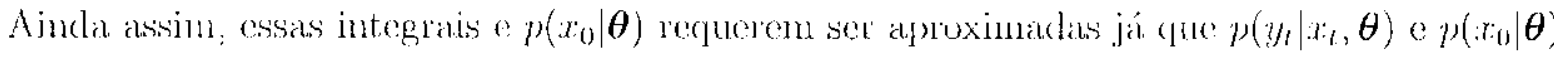
nâo são combinaçoes jincares de uma variável nonnal arlicionado un ruído normal, como no 


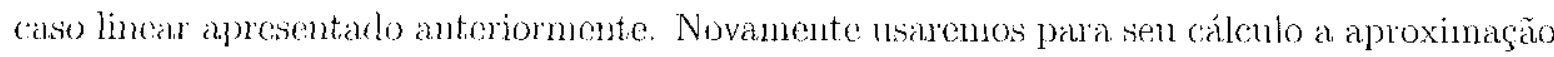
de Lèlplace. Isso nos clá qule?

$$
\left.p\left(y_{t} \mid y_{t-1}, \theta\right) \simeq \sqrt{2 \pi} e^{\psi_{t}\left(y_{h}, \hat{x}_{t}, \theta\right)} \mid I\right)\left.^{2} \psi_{t}\left(y_{t}, \widehat{x}_{t}, \theta\right)\right|^{-1 / 2}
$$

en que

$$
\psi_{1}\left(y_{t}, x_{t}, \theta\right)=-\log \left(p\left(y_{l} \mid x_{t}, \theta\right) \tilde{p}\left(x_{t} \mid y_{t-1}, \theta\right)\right)
$$

$\widehat{x}_{t}$ é o mínimo da função $\psi_{t}\left(y_{t}, x_{t}, \theta\right)$ com relaçäo a $x_{t}$, o $D^{2} \psi_{t}\left(y_{t}, x_{t}, \theta\right)$ denota a derivala

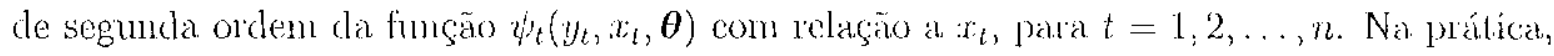
encontrar $\widehat{x}_{f}$ lequer alguna lécnica de estimaçäo nunérica. Sugerimos o nso do algoritno de Newton-Raphson, vejal anondice A.3

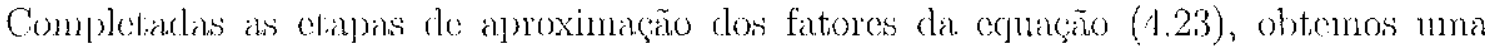
expressão para a verossinilhanģa que não mais depende do conjunto de variáveis lat entes $\boldsymbol{x}$. Mais precisamente, una aproximação para a verossimilhança é kada por

$$
\widetilde{p}(y \mid \theta)=(2 \pi)^{\frac{n+1}{2}} \exp \left\{\sum_{t=0}^{n} \psi_{t}\left(y_{t}, \hat{x}_{t}, \theta\right)\right\} \prod_{t=0}^{n}\left|D^{2} \psi_{t}\left(y_{t}, \widehat{x}_{t}, \theta\right)\right|^{-1 / 2}
$$

\subsection{Abordagem Bayesiana}

Agora (pue obtemos mana aproximagán paua a função de verossimilhança, podemos inserir conhecinmentos a respeito do comportanento de cada paranctro, conlhecimentos a priori. Por cxemplo, no aso dos MVEM, un dos parâmetros deve ser positivo e menor que un. Além disso pesquisas mostran que seu valor lica proxino de un. Novamente temos situagous an que os métodos analíticus nầ são convenientos, sendo necessários métodos de aproximação

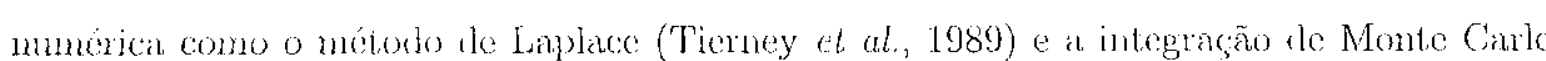
via amostragen por importancia, (veja p. ex. Ripley, 1987).

Pelo teoroma de Bayes a densidade a posteriori é dlakla por

$$
p(\theta \mid \boldsymbol{y})=\frac{p(\boldsymbol{y} \mid \theta) p(\boldsymbol{\theta})}{p(\boldsymbol{y})},
$$

am que $p(\boldsymbol{y})=\int p(\boldsymbol{y} \mid \boldsymbol{\theta}) p(\boldsymbol{\theta}) d \boldsymbol{\theta}$ é a clensidade narginal de $\theta$. No nosso caso, ela é aproximadamente proporcional a

$$
\widetilde{p}(\theta \mid y) \propto \widetilde{p}(y \mid \theta) p(\theta)
$$


en que $\tilde{p}(y \mid \theta)$ ó clatla pela expressão om (4.41). Devemos então maximizar a posterioni aproximada com relacäo ao vetor de paranetros $\theta$, ou seja, encontrar sua moda. Para essa tarefa temos, em geral, a necessidade do uso de métodos de estimação mumérica devido a complexidale dal funcuaro.

O) trabalho de Meyer el al. (2003) triata da forna canònica de Kim (veja equaços (1.23) (1.24)) e cmprega a técnica antomatic differentiation (AD), um conjunto de algoritmos computacionais para a oltenção numórica das derivalas do fumçös näo-lineares intratúveis explicitanente, no uso do algoritmo quase-Newton. Alén disso, sugere o uso do software AD

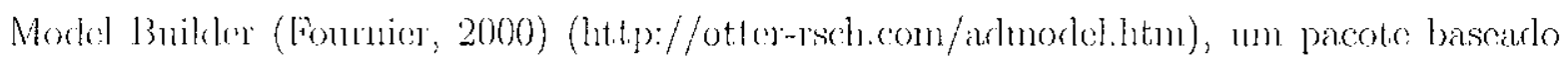

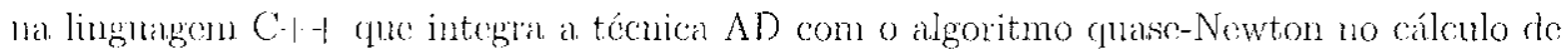
múnimos de funcõoses, para minimizar $\log (\tilde{p}(0 \mid y))$.

O 1180 do algoritmo de Metropolis-Hastings (Itastings, 1970) também é uma alternativa pala encontrar a moda a posteriori. No entanto, sabomos que a oficiência desse algoritmo pode ser comprometida por mu má escolha da densidade geradora. Quanto najo próxima

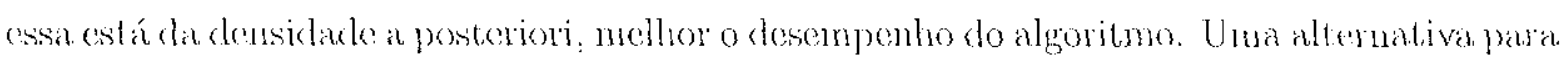

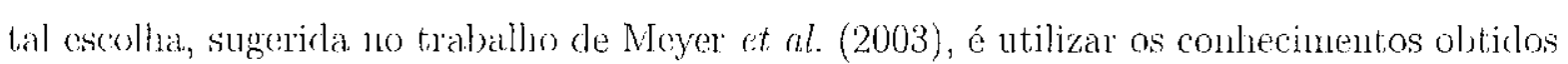

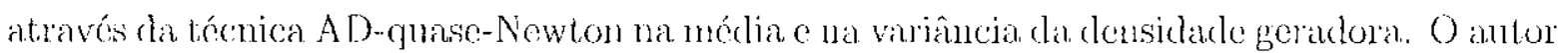
afirma que essa combinaño de recursos resulta numa técuica MCMC bastante flexível, eficaz o alequalia aos MVE.

Vale destacar que o uso da $\Lambda \mathrm{D}$ não ó jndisponsável. Una vez que a posteriori foi aproxi-

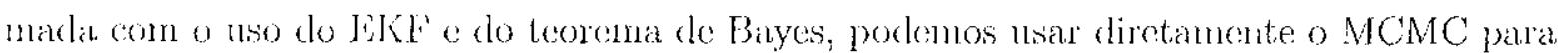
obcer o velor de parinotros estimado.

\subsection{Modelo I}

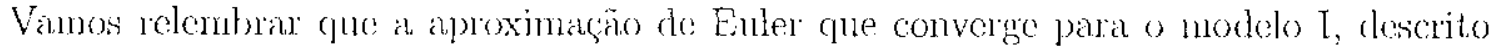

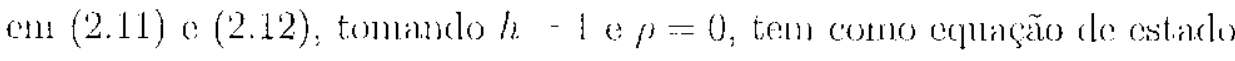

$$
u_{t+1}=\nu_{1}+\beta\left(\alpha-u_{t}\right)+\gamma \tilde{Z}_{t}
$$

parat $t-0,1, \ldots, n$. Estabelecendo condição inicial $\nu_{0}$ tal que

$$
\nu_{0} \sim \mathcal{N}\left(\alpha, \frac{\gamma^{2}}{1 \cdot(1-\beta)^{2}}\right) .
$$




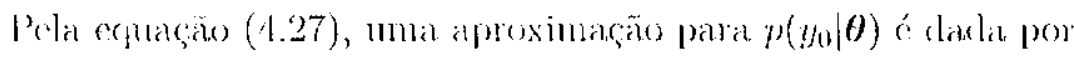

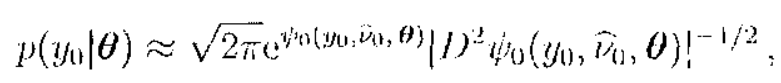

conl

$$
\begin{aligned}
& \psi_{0}\left(y_{0}, \nu_{0}, \theta\right)=\frac{1}{2} \log \left(2 \pi 0^{\nu_{0}}\right) \cdot+\frac{1}{2}-\frac{\left(y_{10}-\mu+e^{\nu_{0}} / 2\right)^{2}}{\mathrm{e}^{\nu_{0}}}+\frac{1}{2} \log \left[\frac{2 \pi \gamma^{2}}{1-(1-\beta)^{2}}\right] \\
& \left.+\frac{1}{2} \frac{(1}{2 \gamma^{2}} \frac{\beta)^{2}}{(\nu / 0}-a\right)^{2}
\end{aligned}
$$

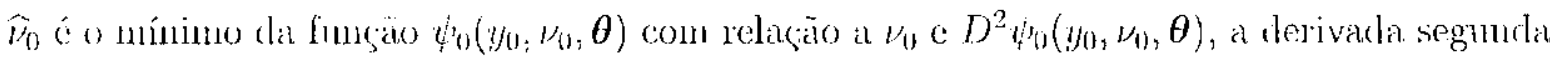

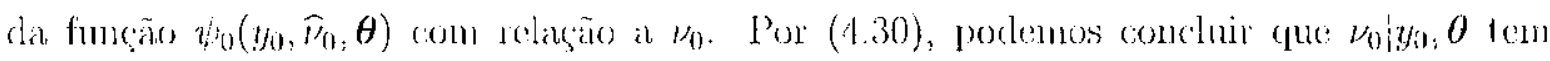

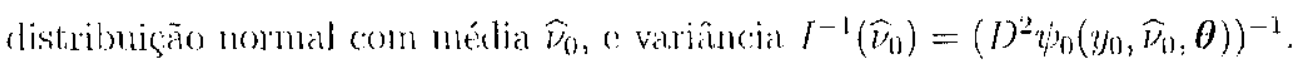

Seguindo a mesma notaçño dacla no início deste capílulo temos

$$
C_{t}\left(\nu_{t-1}\right)-\nu_{t-1} \mid \beta\left(\alpha-\cdots \nu_{t-1}\right)
$$

$$
a\left(\nu_{l-1}\right)=1-\beta
$$

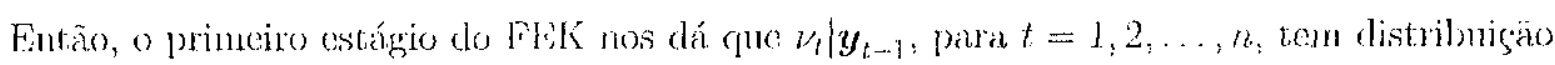

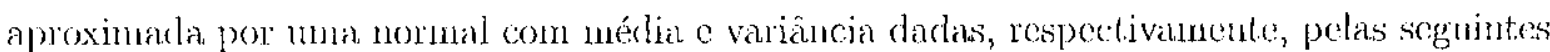
equlaçöes

$$
\bar{\nu}_{l 1-1}=\bar{u}_{t-1}+\beta\left(\alpha-\bar{\nu}_{t-1}\right)
$$

$$
P_{i l t-1}=(1 \cdots \beta)^{2} l^{1}\left(\hat{u}_{t-1}\right) i \cdot \gamma^{2} .
$$

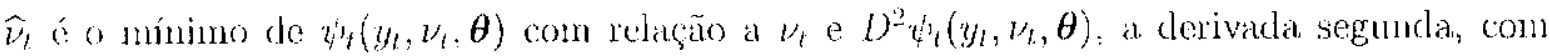

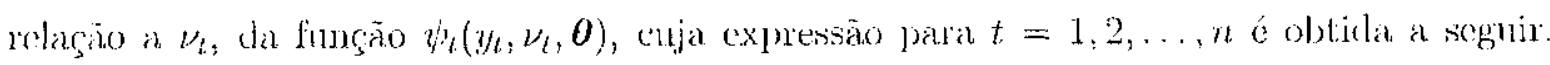

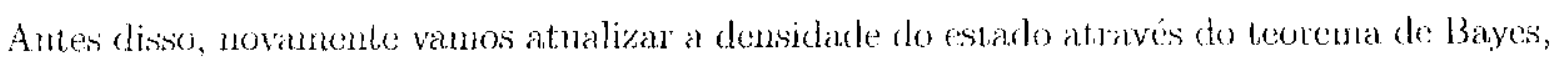

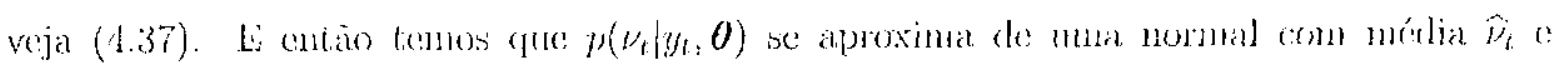
variancia $1^{-1}\left(b_{l}\right)$.

Como (em $(1.39)$ tenos

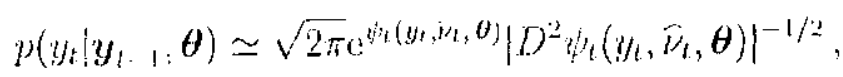


enil cile.

$$
\psi_{t}\left(y_{t}, \nu_{t}, \theta\right) \cdots \frac{1}{2} \log \left(2 \pi e^{\nu_{t}}\right)+\frac{1\left(y_{t}-\mu+\mathrm{e}^{\nu_{t}} / 2\right)^{2}}{2}+\frac{1}{2} \log \left(2 \pi P_{t \mid t-1}\right)+\frac{1}{2 P_{t \mid t} 1}\left(\nu_{t}-\widehat{\nu}_{t, t-1}\right)^{2},
$$

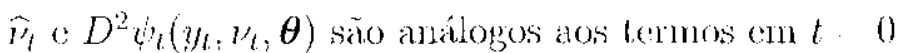

Finalmente podemos expressar a verosimillanga por

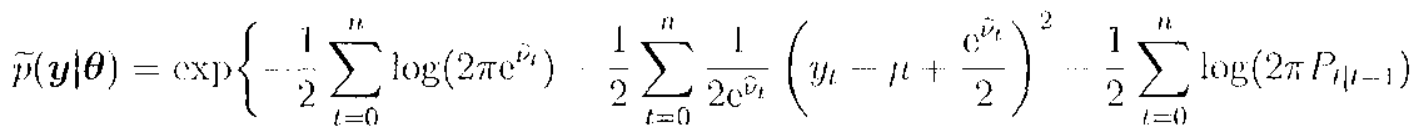

$$
\begin{aligned}
& \left.+\frac{1}{2} \sum_{t-0}^{n} \frac{\hat{p}_{t}-\bar{\nu}_{t \mid t-1}}{P_{t, t-1}^{\prime}}-\frac{1}{2} \sum_{t=0}^{n} \log \left(I\left(\hat{\nu}_{t}\right)\right)\right\} \text {. }
\end{aligned}
$$

\subsection{Modelo II}

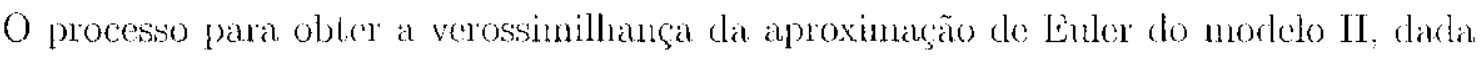
em (2.11) e (2.12), é semellante an caso da segato anterior, pois os processos apenas diferem

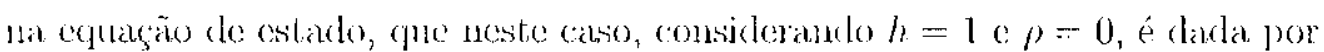

$$
\sigma_{l \mid 1}^{2} \cdots \sigma_{l}^{2}\left|\delta\left(\lambda \cdots \sigma_{l}^{2}\right)\right| \xi \sigma_{l} \tilde{Z}_{l}
$$

para $t=0,1, \ldots, n$. Assmminos condição inicial $\sigma_{0}^{2}$ de forma que

$$
\sigma_{0}^{2} \sim \mathcal{N}\left(\lambda, \frac{\xi^{2}}{1-}\right)
$$

Um aproximação para $p\left(y_{0} \mid 0\right)$, como mostráa (4.27), é dada por

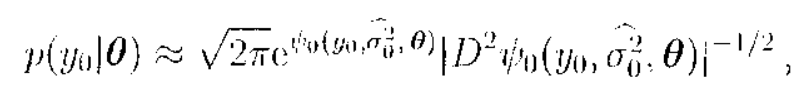

$(0111$

$$
\begin{aligned}
& \psi_{0}\left(y_{0}, \sigma_{0}^{2}, \boldsymbol{\theta}\right)=\frac{1}{2} \log \left(2 \pi \sigma_{0}^{2}\right)+\frac{1}{2}\left(y_{0} \frac{\left.\mu+\sigma_{0}^{2} / 2\right)^{2}}{\sigma_{0}^{2}}+\frac{1}{2} \log \left[\frac{2 \pi \xi^{2}}{1-(1 \cdots \delta)^{2}}\right]\right. \\
& +\frac{1-(1-\delta)^{2}}{2 \xi^{2}}\left(\sigma_{0}^{2}-\lambda\right)^{2}
\end{aligned}
$$

$\widehat{\sigma_{0}^{2}}$ ó o múnimo da funço $\psi_{0}\left(y_{0}, \sigma_{0}^{2}, \theta\right)$ com relaçño a $\sigma_{0}^{2}$ e $D^{2} \psi_{0}\left(y_{0}, \sigma_{0}^{2}, \theta\right)$, a derivada segmela

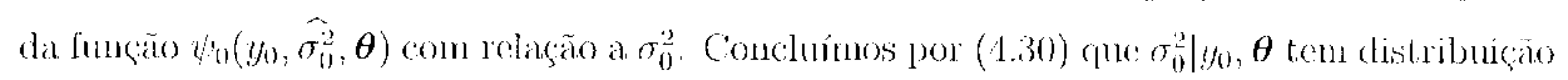
uormal com média, $\widehat{\sigma_{0}^{2}}$, e variância $I^{-1}\left(\widehat{\sigma_{0}^{2}}\right)=\left(D^{2} y_{0}\left(y_{0}, \widehat{\sigma_{0}^{2}}, \boldsymbol{\theta}\right)\right)^{-1}$. 
Com a mesma notaçño nsirla na construça do FFK temos

$$
\begin{gathered}
C_{1}\left(\sigma_{t-1}^{2}\right)=\sigma_{t-1}^{2}+\delta\left(\lambda \cdot \sigma_{t-1}^{2}\right), \\
R_{t}\left(\sigma_{t-1}^{2}\right) \cdots \sqrt{\sigma_{t-1}^{2}} \\
a=1 \quad \delta
\end{gathered}
$$

$$
r_{l}=R_{l}\left(\overline{\sigma_{l}^{2}} l\right)=\sqrt{\overline{\sigma^{2}} l-1}
$$

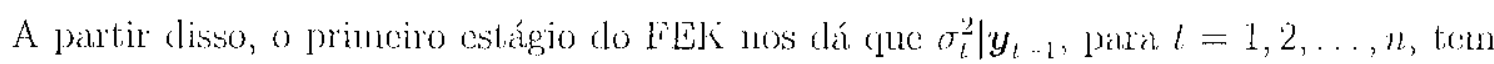

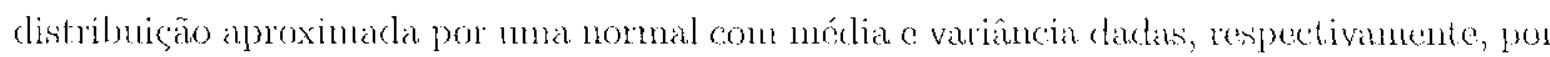

$$
\bar{\sigma}^{2} t \mid t-1=\widehat{\sigma}_{t+\cdots 1}^{2}+\delta\left(\lambda-\widehat{\sigma}_{t-1}^{2}\right)
$$

e

$$
\Gamma_{t_{i} l-1}=(1-\delta)^{2} I^{-1}\left(\widehat{\sigma^{2}} t-1\right)+r_{l}^{2} \xi^{2}
$$

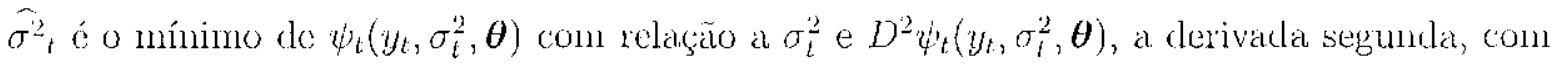

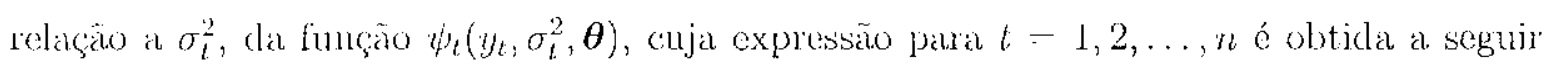

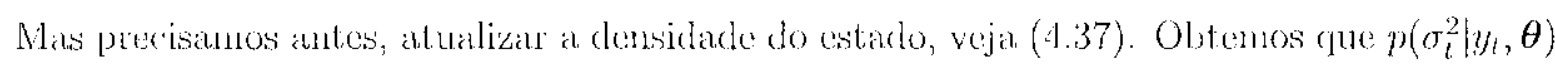

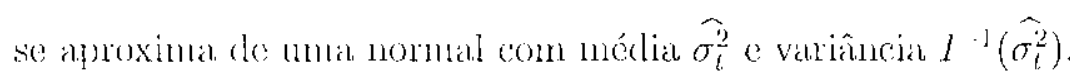

E pela alproximaģio de Laplace, cono en (4.39), temos

$$
p\left(y_{l} \mid y_{l-1}, \theta\right) \sim \sqrt{2 \pi} c^{\psi_{h}\left(y_{t}, \widehat{\sigma_{t}^{2}}, \theta\right)}\left|D^{2} \psi_{l}\left(y_{l}, \widehat{\sigma_{l}^{2}}, \theta\right)\right|^{-1 / 2},
$$

ang quo

$\psi_{t}\left(y_{t}, \sigma_{t}^{2}, \boldsymbol{\theta}\right)=\frac{1}{2} \log \left(2 \pi \sigma_{t}^{2}\right)+\frac{1}{2} \frac{\left(y_{t} \cdots \mu+\sigma_{t}^{2} / 2\right)^{2}}{\sigma_{t}^{2}}+\frac{1}{2} \log \left(2 \pi P_{t \mid t-1}\right)+\frac{1}{2 \Gamma_{t \mid t}+1}\left(\sigma_{t}^{2} \quad \hat{\sigma}^{2} \mid t-1\right)^{2}$,

$\widehat{\sigma_{t}^{2}}$ é o mínino e $D^{2} \psi_{1}\left(y_{h}, \sigma_{1}^{2}, \theta\right)$ a derivadla segunda da função $\psi_{h}\left(y_{h}, \sigma_{1}^{2}, \theta\right)$, ambos com relaçăo a $\sigma_{i}^{2}$.

A verossimilhança para o modelo Il discretizado fica expressin por

$$
\begin{aligned}
& \tilde{p}(y \mid \theta) \cdots \operatorname{ex})^{2}\left\{-\frac{1}{2} \sum_{t=0}^{n} \log \left(2 \pi \widehat{\sigma_{i}^{2}}\right)-\frac{1}{2} \sum_{t=0}^{n} \frac{1}{2 \widehat{\sigma_{t}^{2}}}\left(y_{t}-\mu+\frac{\widehat{\sigma_{t}^{2}}}{2}\right)^{2} \cdots \frac{1}{2} \sum_{t=0}^{n} \log \left(2 \pi P_{t \mid t-1}\right)\right. \\
& \left.+\frac{1}{2} \sum_{l=0}^{n} \frac{\widehat{\sigma_{i}^{2}}-\overline{\sigma^{2}} t i t-1}{P_{t \mid t \cdots 1}} \cdots \frac{1}{2} \sum_{i=0}^{n} \log \left(I\left(\hat{\sigma_{i}^{2}}\right)\right)\right\} .
\end{aligned}
$$




\subsection{Conclusão}

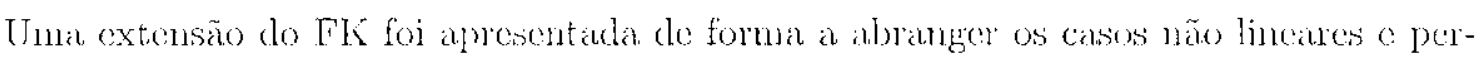

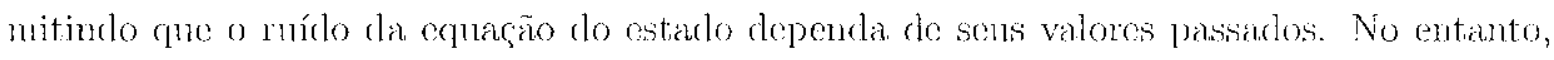
essa abordanem não despreza a hipótese de nomalidade do eno da ontra equagio. Isso nos

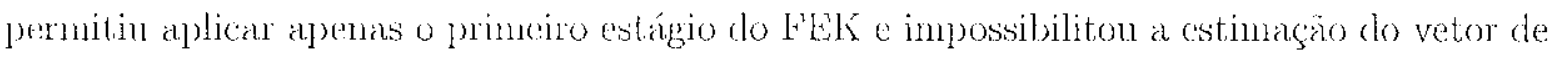
estatolos.

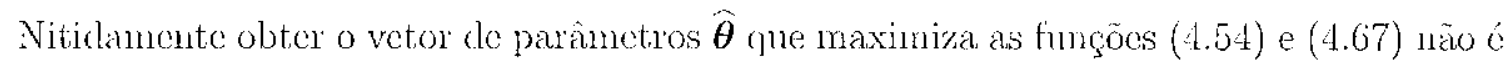

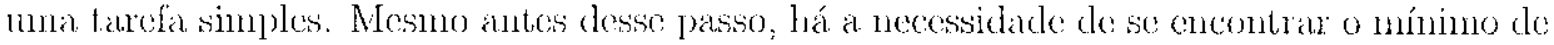
ıma funçăo não-lincar, nsado na aproximagão de Iaplace. O algoritmo de Newton-Raphson,

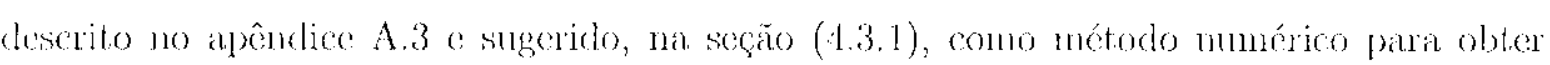
tal múnino, reguen o nso de clerivadas, que muitas vezes precisan tanubén ser estimadas

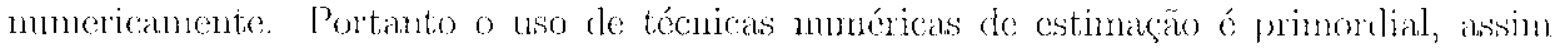
cono sua inplenentação compulakional. Sugerimos para suprir a parte final do problema, o algoritmo de Metropolis Hastings e o uso da inferencia bayesiana 


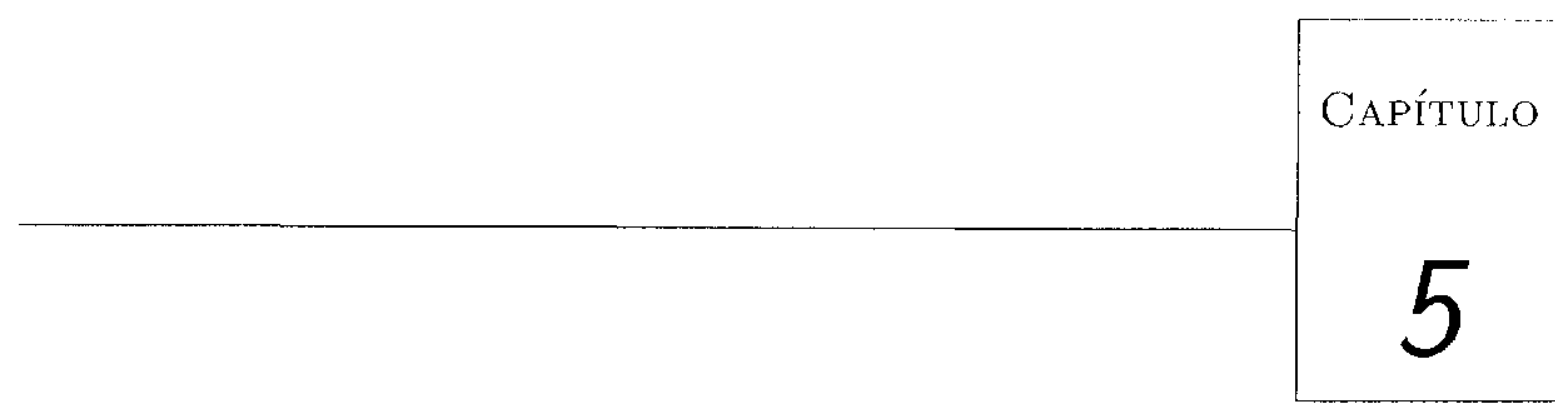

\section{Resultados}

Neste capítulo apresentarenos resultados empíricos para diferentes conjuntos de parâmetros Utilizaremos par'c isso o modelo descrito em (3.35) e (3.36), o qual chamaremos de modelo invariante no tempo (MTT) e seguiremos os procedinentos descritos nas subseções $3.2 .1 \mathrm{e}$ 3.2 .2

\subsection{Abordagem da Verossimilhança}

Utilizando um vetor de parâmetros "verdádeiro" $\theta$, foran gerados 800 dados e descartada a prineira metade deles, para evitar dados enviesados pelo pahpito inicial, servindo-nos como amostra os 400 restantes. Depois tratamos o vetor de parânetros cono desconliecido e utilizarros o método descrito na seção 3.2.1 para estimá-lo. No algoritmo de MetropolisHastings tomanıs como geradora para os parâmetros $a_{1} b, c^{*}$; $l$ e $\gamma^{2 *}$ distribuições normais. Através do software MATY AB, gerannos 1 cadeia com 60000 itcraçōes para os 5 parâmetros. As primeiras 30000 foram desprezadas. A partir das restantes selecionamos de 30 em 30, resultando numa amostra do 1000 valores para a inferència, dada pela méclia dos valores 
Tabela 5.1: Resultados para o MIT A.

\begin{tabular}{cccccc}
\hline \hline Parâmetro & Verdadciro & Estimativa & DP & TA (\%) & CG \\
\hline$a$ & 2.5 & 2.1012 & 0.0872 & 36.9683 & 1.2071 \\
$b$ & -0.8 & -0.7714 & 0.3183 & 77.1067 & -0.6782 \\
$c$ & 0.9 & 0.8871 & 0.0121 & 6.1233 & 0.6162 \\
$d$ & 0.5 & 0.5628 & 0.0613 & 13.7250 & 0.1789 \\
$\gamma^{2}$ & 1.0 & 1.0635 & 0.0903 & 9.7117 & -0.8350 \\
\hline
\end{tabular}

Tabela 5.2: Resultados para o MIT B.

\begin{tabular}{cccccc}
\hline \hline Parâmetro & Verdadeiro & Estimativa & DP & TA (\%) & $\mathrm{CG}$ \\
\hline \hline$a$ & 3.0000 & 2.6743 & 0.1307 & 30.4817 & -1.0728 \\
$b$ & 0 & 0.0941 & 0.1038 & 80.1117 & 0.4750 \\
$c$ & 0.7 & 0.6812 & 0.0403 & 15.6833 & -0.5081 \\
$d$ & 0 & -0.0132 & 0.0523 & 34.5700 & 1.1984 \\
$\gamma^{2}$ & 1.0 & 1.2191 & 0.1071 & 9.1500 & 1.3848 \\
\hline
\end{tabular}

selecionados. A convergência da cadeia foi avaliada usando-se o critério de Goweke (Geweke, 1992). Seu valor deve ser, em módulo, menor do que 2 para indicar que lá convergência dla cadeia, veja apêndice A.4.

Os resultaklos estão sumarizados para dois casos, A e B, nas tabelas 5.1 e 5.2, juntamente con os valores verdadeiros de cada parâmetro, o desvio padrão (DP') da scleygão, a taxa de

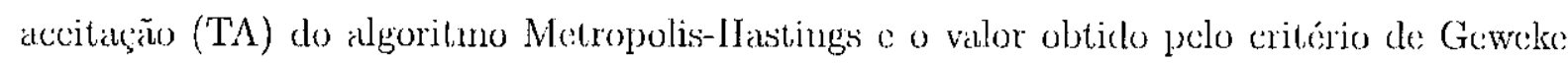
(CG). Nas figunas 5.1 e 5.3 são apresentados os histogramas das donsidandes das amostras selecionadas, respectivamente do MT A e B, para cada parânetro. E os gráficos dos 50\% íltimos valores amostrados são aprescentados nas figuras 5.2 e 5.4, en que podemos analisar à convergêucia de cada parânetro. 

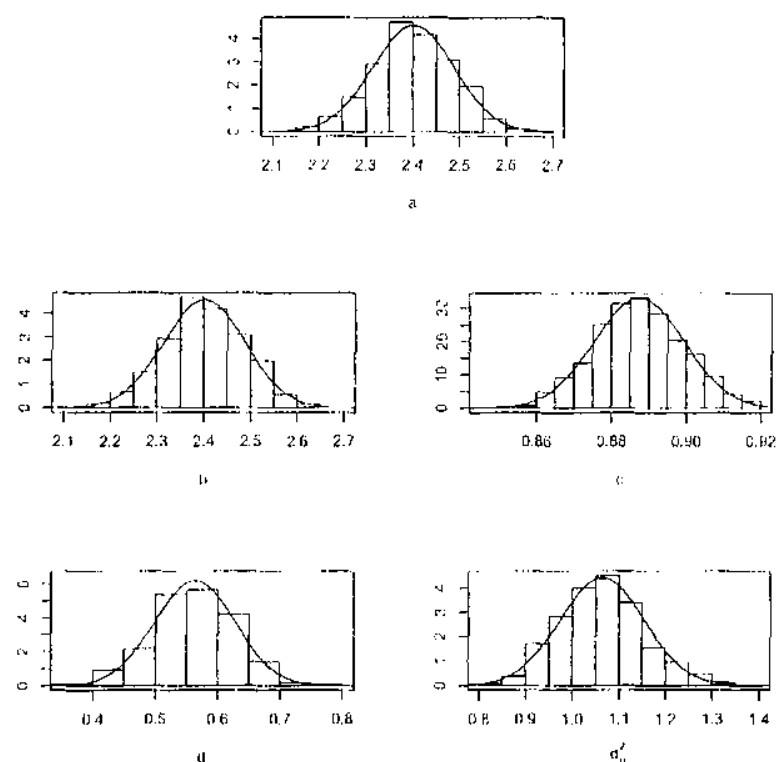

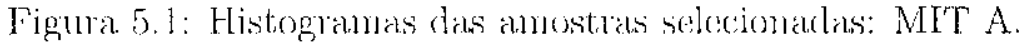
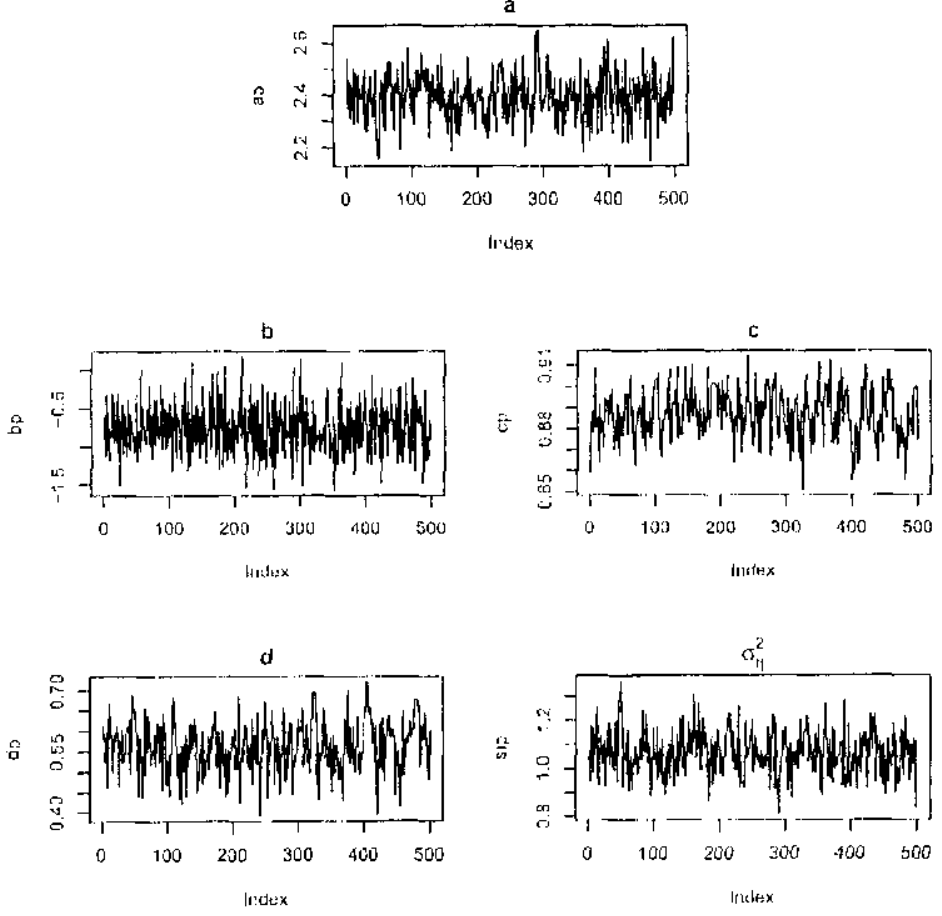

Figura 5.2: Gráticos das anostras selecionadas: MIT $\Lambda$. 

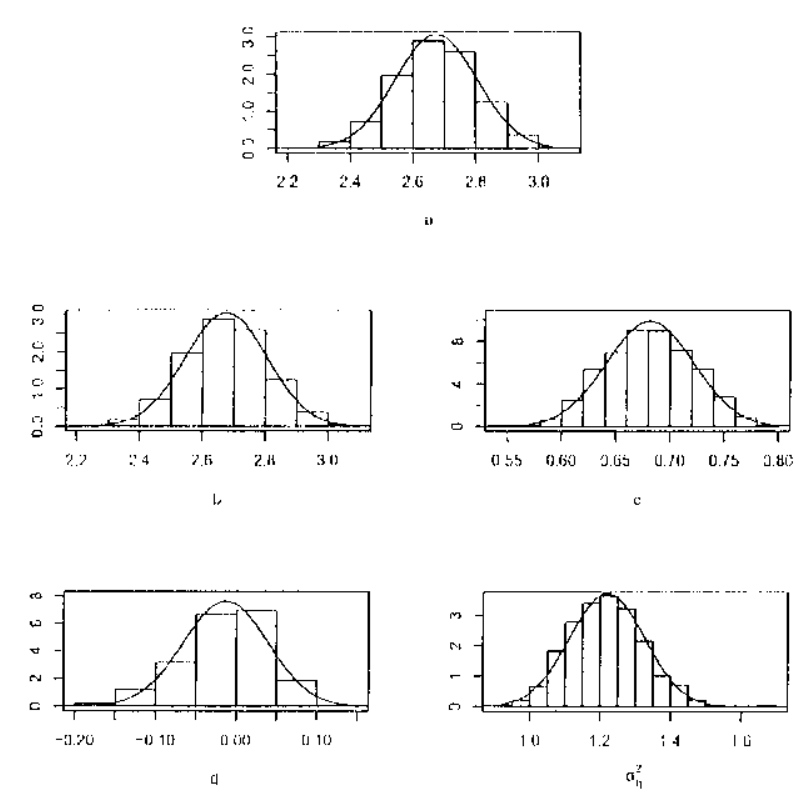

Figma 5.3: Histogramas das amostats selecionadan: Ml'T 13.
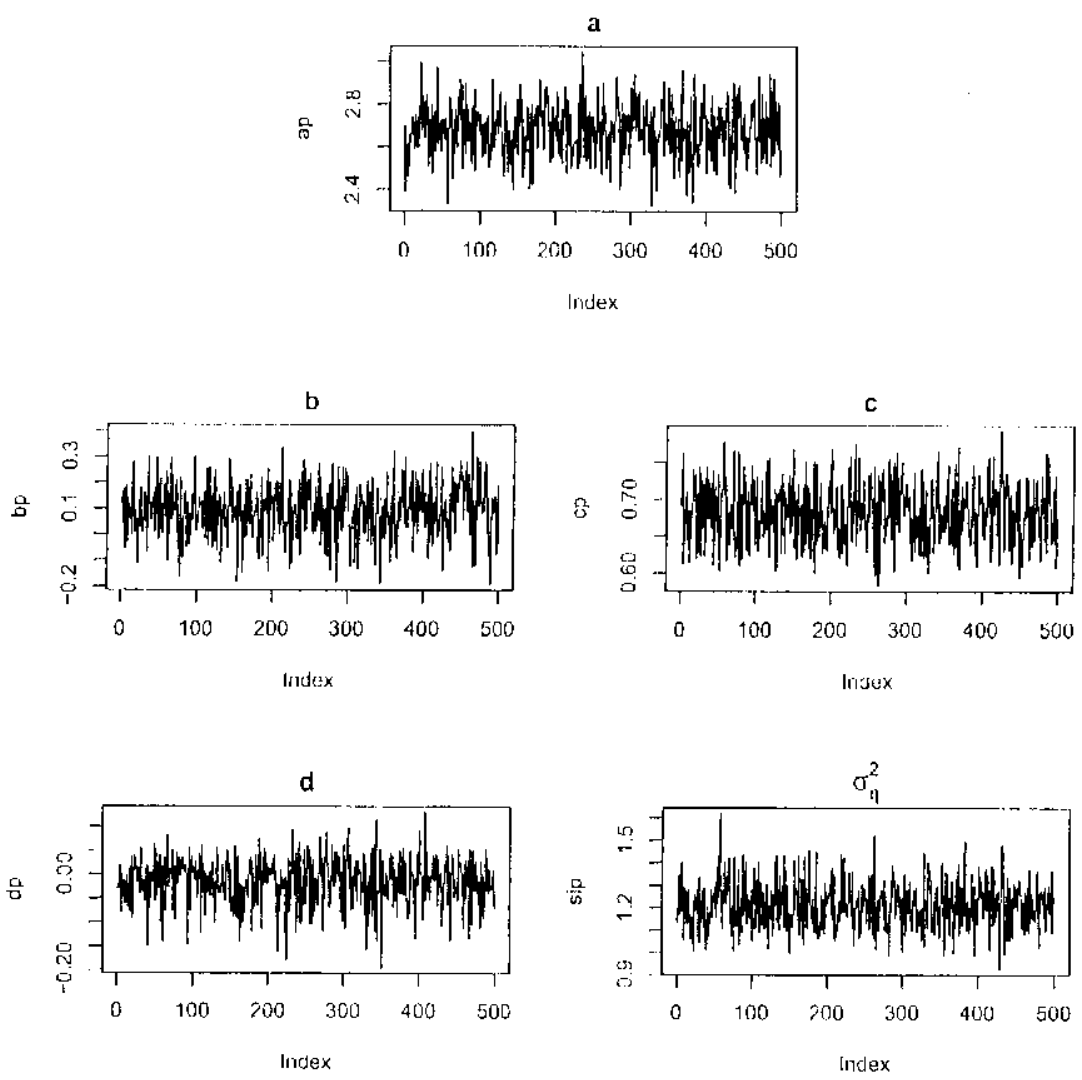

Figura 5.4: Gráficos das amostras selecionadas: MIT B 
Tabcla 5.3: Resultados para o MIT A bayesiano.

\begin{tabular}{cccccc}
\hline \hline Parâmetro & Verdadciro & Estimativa & DP & TA (\%) & CG \\
\hline \hline$a$ & 2.5 & 2.4448 & 0.1543 & 31.8933 & -1.5390 \\
$b$ & -0.8 & -0.7417 & 0.2294 & 90.7133 & 0.0050 \\
$c$ & 0.9 & 0.8859 & 0.0084 & 59.7883 & 1.7412 \\
$d$ & 0.5 & 0.5875 & 0.0586 & 41.9217 & -0.1240 \\
$\gamma^{2}$ & 1.0 & 1.0585 & 0.1402 & 29.3733 & 0.6164 \\
\hline
\end{tabular}

Tabela 5.4: Resultados para o MIT B bayesiano.

\begin{tabular}{cccccc}
\hline \hline Parâmetro & Verdadeiro & Estimativa & DP & TA (\%) & CG \\
\hline \hline$a$ & -1.0 & 2.4012 & 0.0872 & 36.9683 & 1.2071 \\
$b$ & 0.4 & -0.7714 & $0.3183 x$ & 77.1067 & -0.6782 \\
$c$ & 0.7 & 0.8874 & 0.0121 & 6.1233 & 0.6162 \\
$d$ & 0 & 0.5628 & 0.0643 & 13.7250 & 0.1789 \\
$\gamma^{2}$ & 0.25 & 1.0635 & 0.0903 & 9.7117 & -0.8350 \\
\hline \hline
\end{tabular}

\subsection{Abordagem Bayesiana}

Foram usados os mesmos dadus gerados para os casos A e B da seção anterior com o procedimento descrito na seção 3.2.2. As médias das prioris foram consideradas como sendo a estimativa do parâmetro dada pela abordagem de verossimilhança. Para os parâmetros $a, b, c^{*}, d$ e $\gamma^{2 *}$, tomanos como geradora do algorimo de Metropolis-Hastingses distribuiçócs normais. Também nessa abordagem, geramos 1 cadeia com 60000 iterações. Descartamos as primeiras 30000 e, das restantes fizemos uma scleção com passo de $30 \mathrm{em} 30$. Ficamos com uma amostra de 1000 valores para a inferência qque se den pela média da amostra selecionada para cada parâmetro. Utilizamos o crilério de Geweke para avaliar a convergência. Os resultados numéricos estão sumarizados para os casos A e B nas tabelas 5.3 e 5.4 , respectivamente. Já os resultados gráficos do MIT A estão nas figuras 5.5 e 5.6, e os do MIT B, nas figurats 5.7 c 5.8 . 

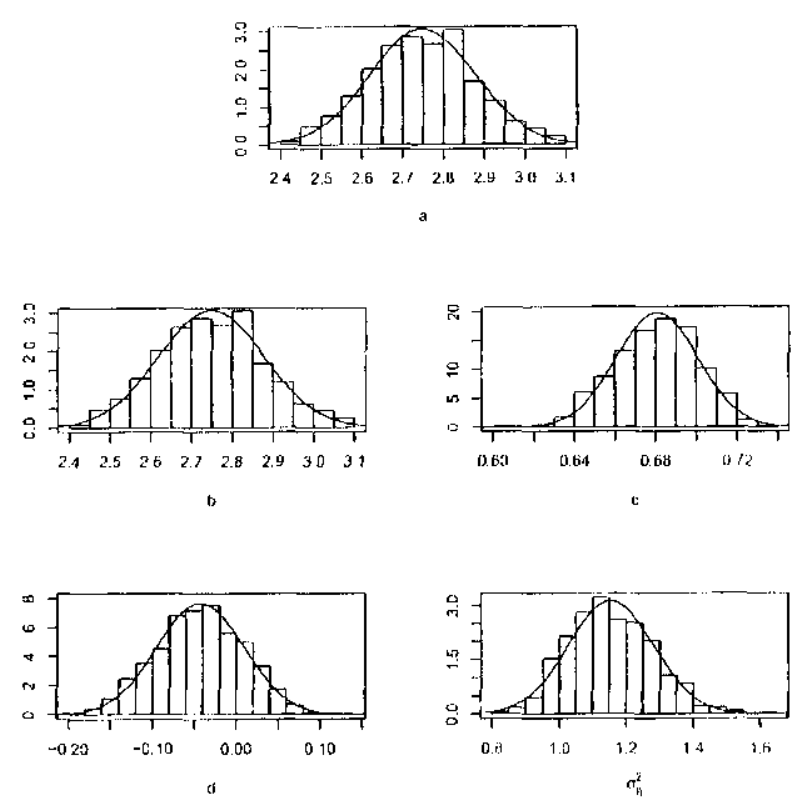

Figura 5.5: Histogranas das anostras selecionadas: MTT A bayesiano.
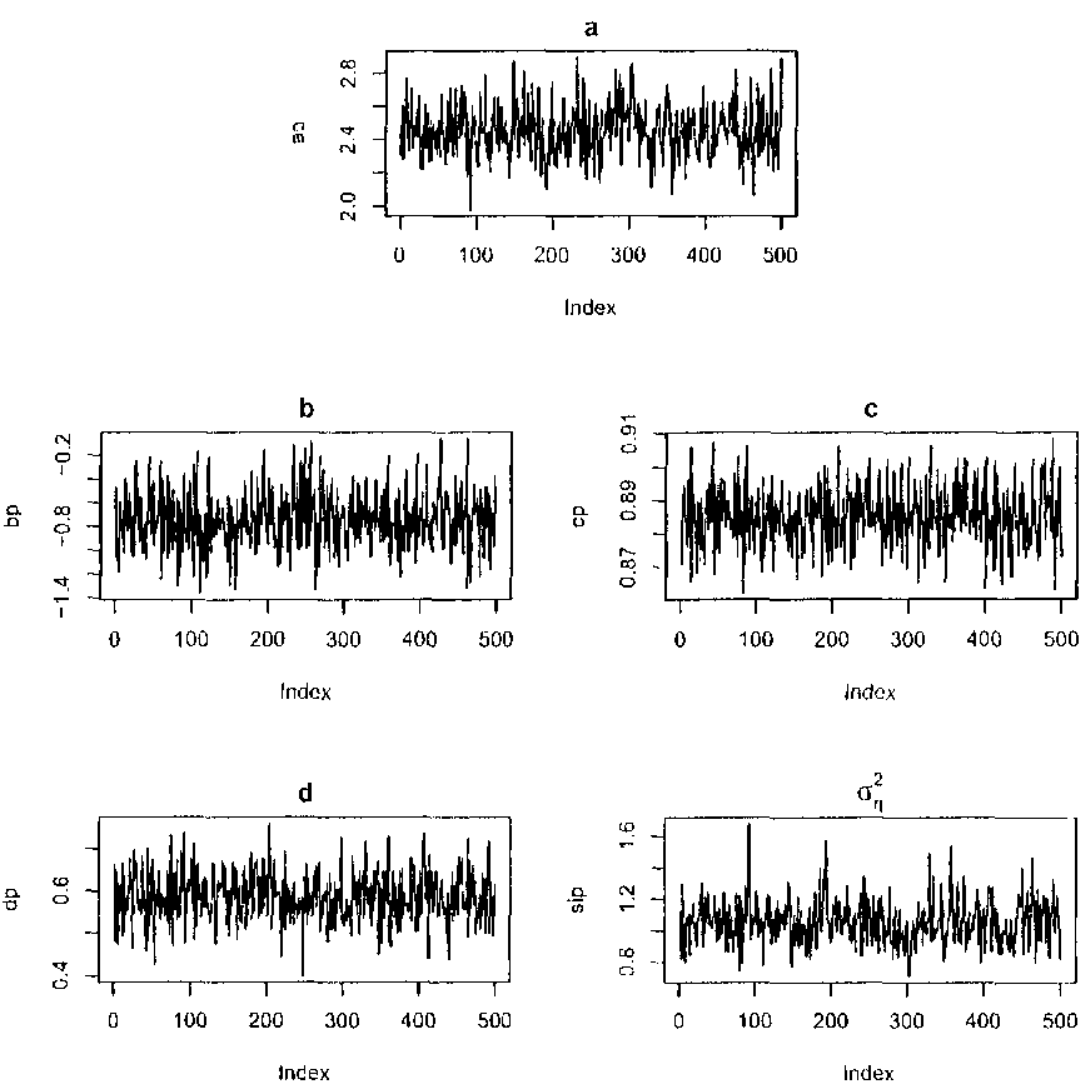

Figura 5.6: Gráficos das amostras selecionadas: MIT A bayesiano. 

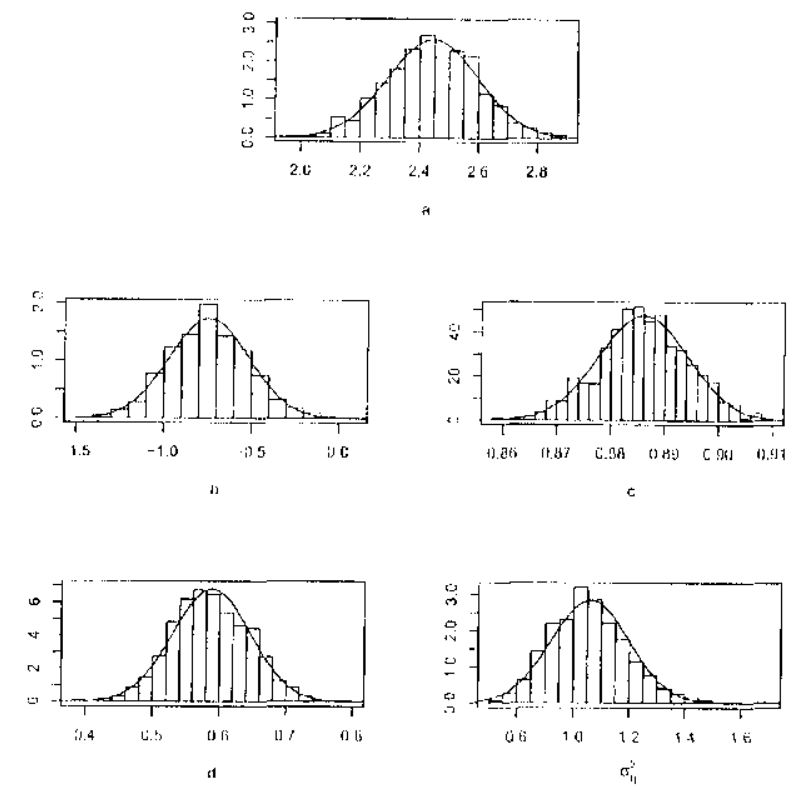

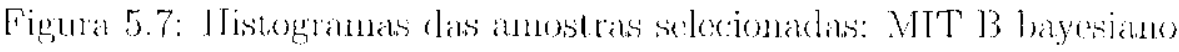
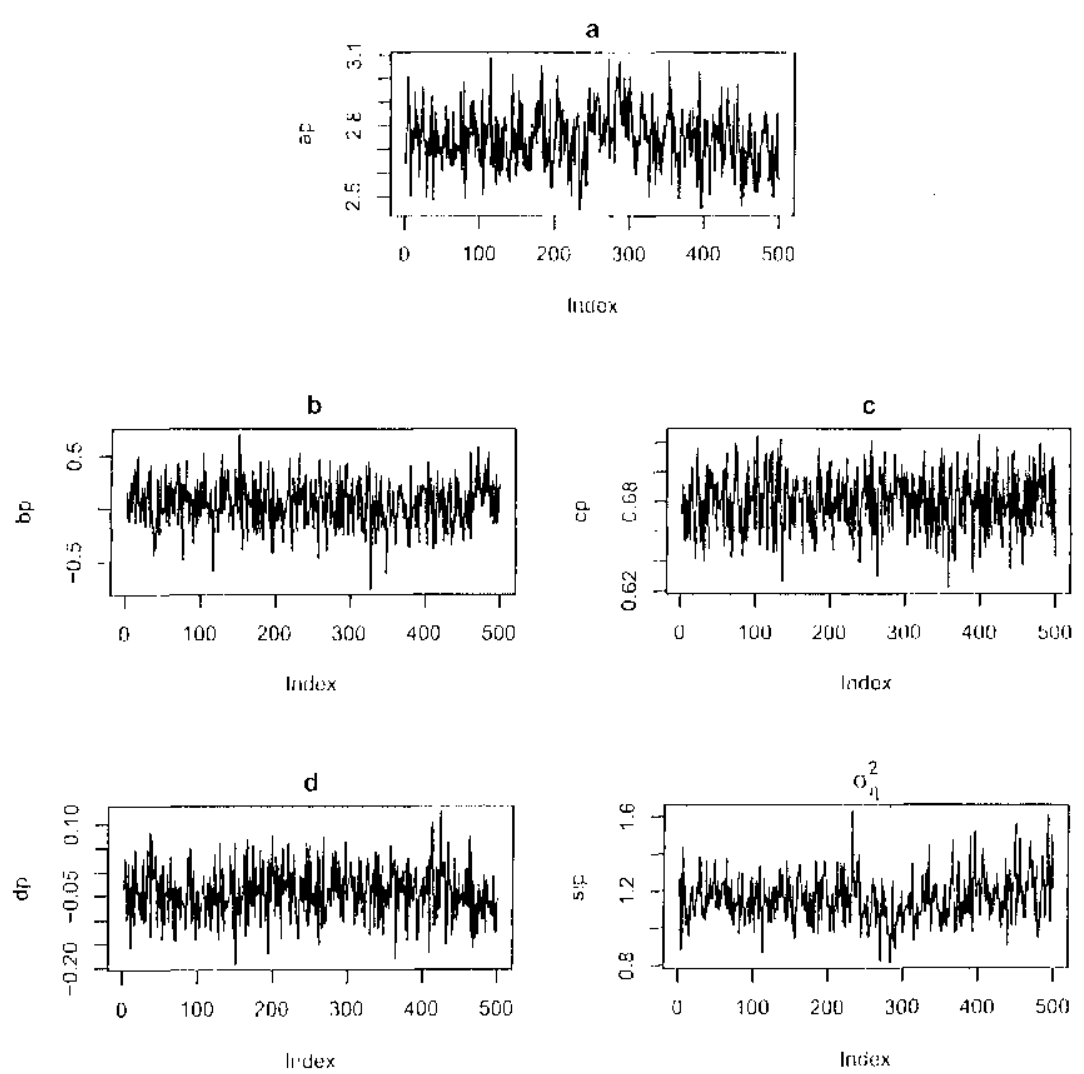

Figura 5.8: Gráficos das amostras selocionadas: MIl B bayesiano. 


\subsection{Conclusão}

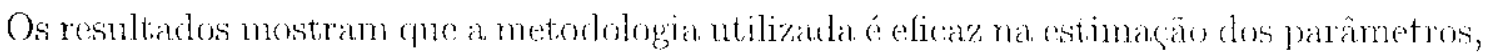

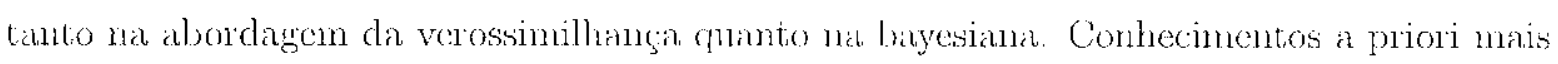
esperílicos podem diminuir o tempo computandomal. 


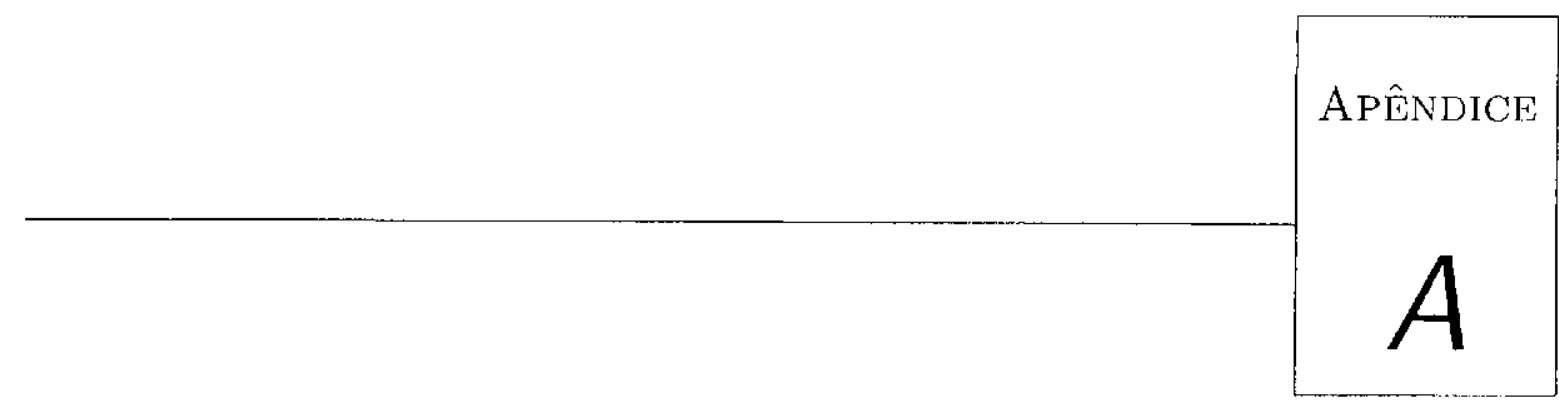

Métodos Numéricos

Neste apêndice apresentaremos alguns métodos numéricos citados neste trabalho. Para mais detalhes a respeito das duas primeiras seçoes sugerimos Press (1989) e Gauneminan (1997), e Milne (1953), da últinna.

\section{A.1 MCMC}

O objetivo dos métudos de Monte Carlo com cadeia de Markov (MCMC) ć gerar uma amosira de uma distribuição $p(\theta)$, para $\theta=\left(\theta_{1}, \theta_{2}, \ldots, \theta_{k}\right) \in \mathbb{R}^{k}$, que não pode ser gerada diretamente. A idéia ó simular uma candeia de Markov irredutível aperiódica cuja distribuição de cquilíbrio ć a distribuição de interesse $p(\theta)$. Se o número de simulaçōes é grande, os valores simulados da cadeia podem ser usados como uma base para identificar características da distribuição de interesse, $p(\theta)$.

A seguir damos dois métodos para gerar cadeia de Markov, conuumente utilizados nos diversos trabalhos publicados dentro desse contexto. 


\section{A.1.1 Amostrador de Gibbs}

O amostrador de Gibbs é $m$ método MCMC essencialmente iterativo, cujo núcleo de transição é formado pelas condicionais completas da distribuição $p(\theta)$, cladas por $p\left(\theta_{1} \mid \theta_{2}, \theta_{3}, \ldots, \theta_{k}\right), p\left(\theta_{2} \mid \theta_{1}, \theta_{3}, \ldots, \theta_{k}\right), \ldots, p\left(\theta_{k} \mid \theta_{1}, \theta_{2}, \ldots, \theta_{k-1}\right)$. O algoritmo do processo 0 dado a seguir.

1. inicia com valor $\boldsymbol{\theta}^{(0)}=\left(\theta_{1}^{(0)}, \ldots, \theta_{k}^{(0)}\right)$ c contador de iteração $j=0$;

2. gera

(i) $\theta_{1}^{(j+1)}$ de $p\left(\theta_{1} \mid \theta_{2}^{(j)}, \theta_{3}^{(j)}, \ldots, \theta_{k}^{(j)}\right)$;

(ii) $0_{2}^{(j+1)}$ de $p\left(\theta_{1} \mid \theta_{2}^{(j)}, \theta_{3}^{(j)}, \ldots, \theta_{k}^{(j)}\right)$;

(k) $\theta_{k}^{(j+1)} \operatorname{de} p\left(\theta_{2} \mid \theta_{1}^{(j)}, \theta_{3}^{(j)}, \ldots, \theta_{k}^{(j)}\right)$;

3. faz $j=j+1$ e volta ao passo 2 até obter a convergência.

Conforme o número de iterações aumentia, a cadeia se aproxima de sua condição de equilíbrio. Desta forma, assume-se que a convergência foi obtida quando a distribuição da amostra estiver arbitranianente: próxima da distribuição de equilíbrio. Para verificar a convergência rlo algoritmo (xisten algumas técnicas. Gelfand \& Smith (1995) sugere o nso de técnicas gráficas. Já o critério de Geman-Rubin (Gelman \& Rubin, 1992) e o critério de Gewcke (Gewoke, 1992) utilizann mma análise estiatística dos dados da anostra gerada.

\section{A.1.2 Metropolis-Hastings}

Quando as distribuições condicionais não são facilmente identificadas, ou seja, não possuem formas padrões (normal, beta, gama, entre outras), podemos utilizar, para gerar as amostras das caleias de Markov, o alyoritmo Metropolis-Hastings, ou métodos de amostragem por importância, Metropolis et al. (1953)

Suponhamos que querenos gerar amostras de uma densidade não-reguar $p\left(\theta_{i} \mid \theta_{1}, \ldots, \theta_{i-1}, \theta_{i+1}, \ldots, \theta_{k}\right) \doteq p\left(\theta_{i} \mid \theta_{(-i)}\right)$. Devermos definir um núcleo de transição $q\left(\theta, \theta^{*}\right)$ da distribuição $p(\theta)$ que represente $p\left(\theta_{\imath} \mid \theta_{(-i)}\right)$ transformando $\theta$ em $\theta^{*}$. O algoritmo de Metropolis-Hastings pode ser esquematizado como segne 
1. inicia con valor $\theta^{(0)}$ e contador de iteraşã̃o $j=0$;

2. $\theta^{*}$ de acordo com o núcleo de transição $q\left(\theta^{(j)}, \theta^{*}\right)$;

3. atualiza $\theta^{(j)}$ por $\theta^{(j+1)}=\theta^{*}$ com probabilidade

$$
p=\min \left\{1, \frac{p\left(\boldsymbol{\theta}^{*}\right) q\left(\boldsymbol{\theta}^{(j)}, \boldsymbol{\theta}^{*}\right)}{p\left(\boldsymbol{\theta}^{(j)}\right) q\left(\boldsymbol{\theta}^{*}, \boldsymbol{\theta}^{(j)}\right)}\right\},
$$

ou seja, permanece com $\theta^{(j)}$ com probabilidade $1-p$;

4. faz $j=j+1$ e repete os passos de 2 e 3 até conseguir una distribuição estacionária.

Observação A.1. Algunas considoraçōes podem ser feitas, como seguem

(i) O algoritmo do Metropolis-Hastings é especificado pela sia densidade candidata pared geração $q(x, y)$;

(ii) se um valor candidato é rejeitado, o valor atıal é considerado na próxima etałpa;

(iii) o cálculo da probabilidade de transição $p$, em ( $\Lambda .1)$, não depende da constante normalizadora;

(iv) se a densidade candidata para geraçĩo dass amostras é simétrica, ou seja, $q(x, y)=$ $q(y, x)$, a probalbilidade de movimento se redur a $p\left(\theta^{*}\right) / p\left(\theta^{(j)}\right)$. Assim, se $p\left(\theta^{*}\right)>$ $p\left(\theta^{(j)}\right)$, a cadcia se move para $\theta^{*}$; caso contrário, move-se para $\theta^{*}$ com probabilidade $p\left(\boldsymbol{\theta}^{*}\right) / p\left(\boldsymbol{\theta}^{(j)}\right)$. Em outras palavras podemos dizer que unz salto na direşão "abcendente" é sempre aceito, enquanto que um salto na direção "descendente" é aceito com uma certa probabilidade.

\section{A.2 Aproximação de Laplace}

A aproximação de Laplace é um método numérico que aproxima assintoticamente a distribuição a posteriori de una variável aleatória $y$. Consideremos $x$ uma variável aleatória de densidade $p(x)$ e com $p(y \mid x)$ conhecida. A densidade a posteriori de $y$ com relação a $x$ é dada por

$$
p(y)=\int_{-\infty}^{\infty} p(y \mid x) p(x) d x
$$


Sejam $\psi(y, x)=\cdots \log (p(y \mid x) p(x))$ e $\widehat{x}=\min _{x}\{\psi(y, x)\}$ o mínimo da função $\psi$ cm relação a $x$. Tomemos a expansão $\mathrm{cm}$ sćrie de Taylor de segunda ordem de $\psi$, apenas em relação a $x$, em torno de $\widehat{x}$. Assim

$$
\psi(y, x) \approx \psi(y, \widehat{x})-\frac{1}{2} I^{2} \psi(y, \widehat{x})(x-\widehat{x})^{2}
$$

cm que $D^{2} \psi(y, \widehat{x})=\partial^{2} \psi(y, x) / \partial x^{2}$ calculada em $\widehat{x}$. Então

$$
p(y) \approx e^{\psi \psi(y, \widehat{x})} \int_{-\infty}^{\infty} \exp \left\{-\frac{1}{2} \frac{(x-\widehat{x})^{2}}{\left(-D^{2} \psi(y, \widehat{x})\right)^{-1}}\right\} d x .
$$

Fazendo uma mudança de variávcis obtemos que

$$
p(y) \approx \sqrt{2 \pi} e^{-\psi(y, \widehat{x})}\left|D^{2} \psi(y, \widehat{x})\right|^{-1 / 2}
$$

\section{A.3 Algoritmo de Newton-Raphson}

O) método de Newton-Raphson é um algoritmo recursivo parra encontrar máximos de funções que possuam derivadas de, ao menos, segunda ordem contínuas. A idéia do algoritmo ó utilizar a expansão cm sérics de Taylor até segundia ordem dia função en questão.

Seja $f$ uma função real, com derivadas de primeira e scgunda ordens contínuals. O algoritmo de Newton-Raphson para $f$ é descrito a seguir.

1. inicia com valor $x_{0}$ e contador de iteração $j=0$;

2. enquanto $\left|f^{\prime}\left(x_{j}\right)\right|>\varepsilon$

(i) $x_{j+1}=x_{j}-\frac{1}{f^{\prime \prime}\left(x_{j}\right)} f^{\prime}\left(x_{j}\right)$,

(ii) $j=j+1$,

(iii) $x_{\max }=x_{j}$

3. o valor que maximiza $\int$ localmente é $x_{\max }$.

$\Lambda$ derivada primeira da função deve ser nula no ponto de mínimo. O valor clado a $\varepsilon$ expressa o quanto é permilido que a derivadia difira de zcro.

O algoritmo de Newton-Raphson pode ser facilnuente estcrdicio para funçõos multivariadas. 


\section{A.4 Critério de Gewekc}

O critério de Goweke (Ceweke, 1992) é um método mumerico para avaliar a convergência da quantidacle anostradia, quando esta é gerada utilizando-se apentas una cadeia.

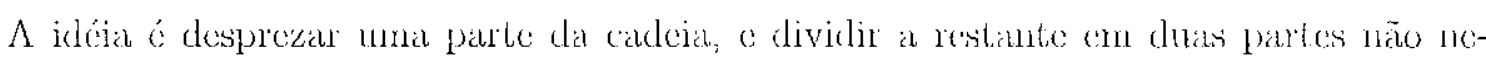

ressarianente ignais. Para cada parte, estiman-se sua média c variancia. Considera-se

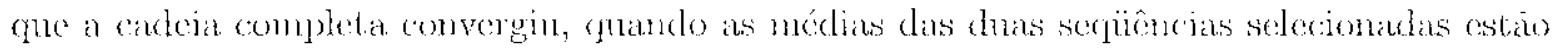
próximas. E jsso ave avalia com un teste de comparaño das médias. Com um erro do $5 \%$, valor do teste deve ser; cm módulo, menor do que 1,96 


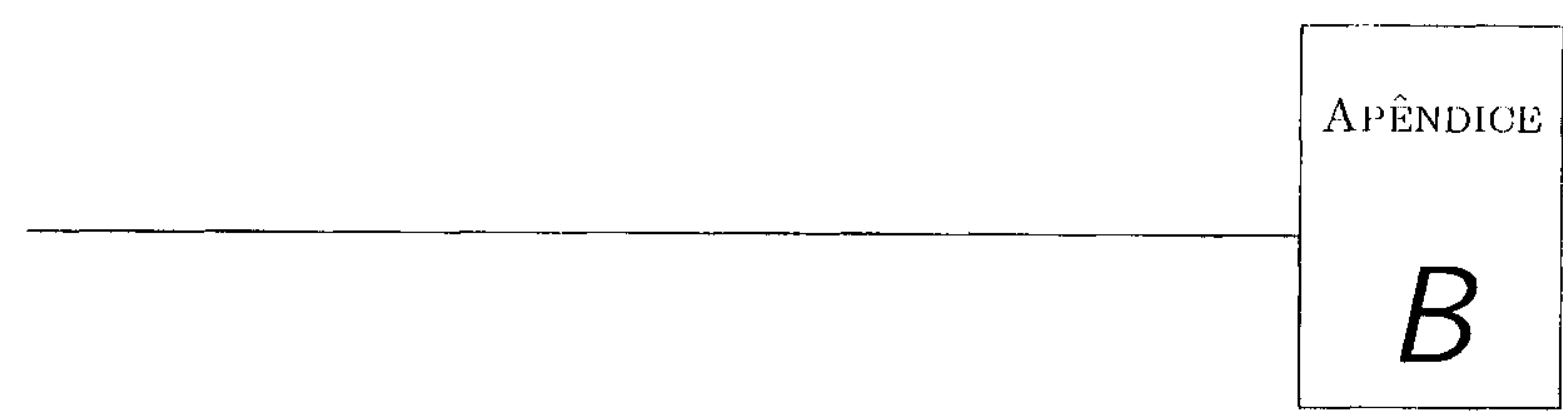

Modelos Discretos

\section{B.1 Modelos ARCH(p)}

O processo ARCH pode ser resumido por

$$
\begin{gathered}
Y_{t}=X_{t-1} \beta \mid Z_{t} \\
Z_{t} \mid \Omega_{t-1} \sim I^{2}\left(0, \sigma_{l}^{2}\right) \\
\sigma_{l}^{2}=\alpha_{0}+\sum_{j=1}^{p} \alpha_{j} Z_{t: 1}^{2},
\end{gathered}
$$

en que $P(\cdot)$ é uma distribuição pararnétrica, usualmente a Nomal ou a $t$-Student, $X_{t}$ denota un vetor de variáveis sxógena, $\beta$ urn vetor de parâmetros desconhecidos e $\Omega_{t-1}$ ó o conjunto de informaçöes obtidas até o instanı, $t-1$, on seja, $\Omega_{t-1}=\left\{Z_{t-1}, Z_{t-2}, Z_{t-3}, \ldots\right\}$.

Dada uma sćrie financeira $S_{t}$ (preço de uma ação, de uma opşão de connpra ou opsăo de venda...) observada em tempo discreto $t_{1}, t_{2}, t_{3}, \ldots$, queremos modelar a volatilidade do

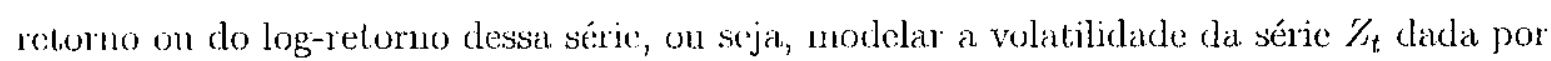

$$
Z_{l}=\log \frac{S_{l}}{S_{l-1}}
$$


Para representar esta série, considcramos $\mathrm{cm}$ (B.1) $\beta=0$, e o processo $Z_{\iota}$ satisfazendo o modelo

$$
\begin{aligned}
Z_{t} & =\sigma_{t} \varepsilon_{l} \\
\sigma_{t}^{2} & =\alpha_{0}+\sum_{j=1}^{p} a_{j} Z_{t-j}^{2},
\end{aligned}
$$

em que $\left\{\varepsilon_{t}, t \geq 0\right\}$ é uma sequiência i.i.d. $\operatorname{com} \mathrm{E}\left(\tilde{\varepsilon}_{t}\right)=0$ e $\operatorname{Var}\left(\varepsilon_{t}\right)=1$.

Por definição $Z_{t}$ é serialmente não correlacionado com média zero e variância condicional $\sigma_{l}^{2}$, uma função do tempo que pode não ser constante. Assunnindo que $\varepsilon_{l} \sim \mathcal{N}(0,1)$ então

$$
Z_{t} \mid \Omega_{t-1} \sim \mathcal{N}\left(0, \sigma_{t}^{2}\right)
$$

A cquação (B.6) pode ser expressada por

$$
\sigma_{t}^{2}=\sigma\left(Z_{t-1}, Z_{t-2}, \ldots, Z_{t-p}, \boldsymbol{\alpha}\right)
$$

cnl que $\alpha=\left(\alpha_{0}, \alpha_{1}, \ldots, \alpha_{p}\right)$ é o vetor de parâmetros desconhecidos.

Como $\sigma_{l}^{2}$ é a variancia condicional, devemos assegurar que $\sigma_{l}^{2}$ seja estritanente positiva para qualquer realização de $Z_{t}$. Para isso, impomos que $\alpha_{0} \geq 0$ e $\alpha_{j}>0, \forall j=1,2, \ldots, p$ em (B.6).

O cálculo da esperança não condicional ć simples

$$
\begin{aligned}
\mathrm{E}\left(Z_{t}\right) & =\mathrm{E}\left[\mathrm{E}\left(Z_{t} \mid \Omega_{t-1}\right)\right]=\mathrm{E}\left[\left(\sigma_{t} \varepsilon_{t} \mid \Omega_{t-1}\right)\right] \\
& =\mathrm{E}\left\{\mathrm{E}\left[\left(\alpha_{0}+\sum_{j=1}^{p} \alpha_{j} Z_{l-j}^{2}\right) \varepsilon_{t} \mid \Omega_{t-1}\right]\right\} \\
& =\mathrm{E}\left[\left(\alpha_{0}+\sum_{j=1}^{p} \alpha_{j} Z_{t-j}^{2}\right) \mathrm{E}\left(\varepsilon_{t} \mid \Omega_{t-1}\right)\right]=0 .
\end{aligned}
$$

Já para calcularmos a variânciá não condicional de um modelo ARCH vamos utilizar a Lei das Esperanças Iterativas. Esta lei sugere que as csperanças das observaçōes correntes ou função dolas, $g\left(Z_{l}\right)$, com respecito as informaçöes disponíveis no tempo $t-J$, podem ser encontradas tomando a esperança condicional da informação no período anterior, $\ell-1$, e cutão tomar a esperança condicional da informação no período anterior a cste, e assim por diante até chegar em $t-J$. Porticuto a esperança de $g\left(Z_{i}\right)$ no tempo $t-J$ pode ser obticla 
por

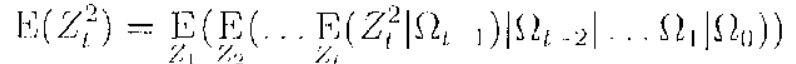

$$
\begin{aligned}
& =\alpha_{0}+\alpha_{n}\left(\sum_{j=1}^{p} \alpha_{j}\right)+\alpha_{0}\left(\sum_{j=1}^{p} \alpha_{j}\right)^{2}+\cdots+\alpha_{0}\left(\sum_{j=1}^{n} \alpha_{j}\right)^{\prime},
\end{aligned}
$$

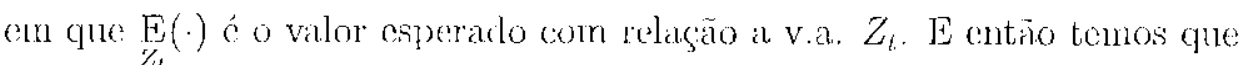

$$
\operatorname{Var}\left(Z_{i}\right)=\mathrm{E}\left(Z_{i}^{2}\right)=\frac{\alpha_{0}}{1-\sum_{j-1}^{p} \kappa_{j}}
$$

Outra forma de demonstrar (B.11) é usando a propricdade descrita na ecplação (B.6). Como devemos ter $\operatorname{Var}\left(Z_{t}\right)>0$ é necessário que $\alpha_{0}>0$ e $\sum_{j=1}^{b} \alpha_{j}<1$. Fista condição é necessária r suficicute para que o processo $\operatorname{ARCH}(p)$ tenlıa covariância cstacionária, veja Engle (1982).

\section{$\operatorname{ARCH}(1)$}

Quindop $=1$ tenus

$$
\sigma_{l}^{2}=\alpha_{0}+\alpha_{1} \gamma_{l-1}^{2}, \quad \alpha_{0}>0, \alpha_{1} \geq 0
$$

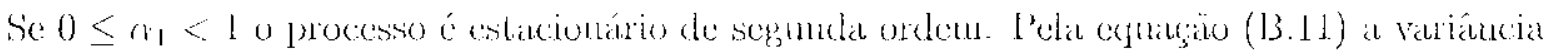
nãu comblicional é dada por

$$
\operatorname{Var}\left(Z_{t}\right) \cdots \frac{\alpha_{0}}{1-\alpha_{1}}
$$

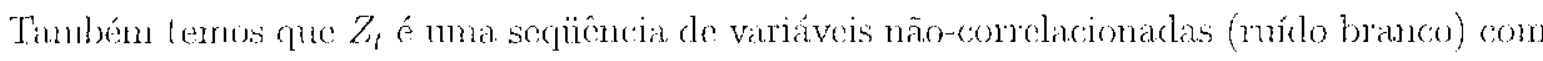
nédia zero e variância $\frac{a_{0}}{1-\alpha_{1}}$. Do fatio,

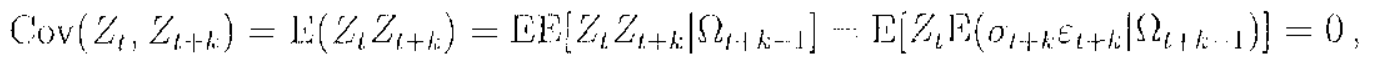

pois $\Omega_{t+k-1}=\left\{Z_{t+k-1}, Z_{t+k-2}, \ldots\right\}$ e $\sigma_{t+k}$ é explicado por $Z_{t+k-1}$. Além disso, E $\left(\varepsilon_{t+k} \mid \Omega_{t ; k-1}\right)=$ 0.

Supunclo $\varepsilon_{1} \sim \mathcal{N}(0,1)$ lichos

$$
\mathrm{E}\left(Z_{l}^{4} \mid \Omega_{t-1}\right)=\mathrm{E}\left(h_{t}^{4} \varepsilon_{l}^{1} \mid \Omega_{t-1}\right)=\left(\alpha_{0}+\alpha_{1} Z_{t-1}^{2}\right)^{2} \mathrm{E}\left(\varepsilon_{t}^{2}\right) \mid \Omega_{t-1}=3\left(\alpha_{01}+\alpha_{1} Z_{t-1}^{2}\right)^{2} .
$$

Assim

$$
\mathrm{E}\left(Z_{t}^{4}\right)=\mathrm{E}\left[\mathrm{E}\left(Z_{l}^{4} \mid \Omega_{l-1}\right)\right]=3 \mathrm{E}\left[\left(\alpha_{0}+\alpha_{1} Z_{t-1}^{2}\right)^{2}\right]=3 \mathrm{E}\left(\alpha_{0}^{2}+2 \alpha_{0}\right)\left(x_{1} Z_{t-1}^{2}+\alpha_{1} Z_{t-1}^{4}\right)
$$


Consicleraudo gue o processo ó estacionário de quartin ordem, temos

$$
\begin{aligned}
& \mathrm{E}\left(Z_{1}^{1}\right)-3\left[\alpha_{10}^{2}+2 \alpha_{0} \alpha_{1} \operatorname{Var}\left(Z_{1}\right)+\alpha_{1}^{2} \mathrm{E}\left(Z_{t-1}^{1}\right)\right]-3 \alpha_{0}^{2}+6 \alpha_{0} \alpha_{1} \frac{\alpha_{0}}{1-\alpha_{1}}+3 \alpha_{1}^{2} \mathrm{E}\left(Z_{1}^{4}\right) \\
& \Rightarrow \quad\left(1-3 \alpha_{1}^{2}\right) \mathrm{E}\left(Z_{1}^{1}\right)=3 \alpha_{0}^{2}+6 \alpha_{0}^{2} \frac{\alpha_{1}}{1-\alpha_{1}} \\
& \mathrm{~F}\left(z_{1}^{1}\right)=\frac{3 r_{0}^{2}}{1-3 r_{1}^{2}}+\frac{\left(6 r_{0}^{2}\right.}{\left(1 \quad 3 r_{1}^{2}\right)\left(1-r_{1}\right)}=\frac{3 r_{1}^{2}\left(1+r_{1}\right)}{\left(1-3 r_{1}^{2}\right)\left(1-r_{1}\right)} .
\end{aligned}
$$

Então, para que momento de (phata ordem seja finito devemos ter $0<r e^{2}<1 / 3$.

Analisando at curtiose de $Z_{l}$

$$
K=\frac{E\left(Z_{1}^{1}\right)}{\left(\operatorname{Var}\left(Z_{1}\right)\right)^{2}} \cdots \frac{3\left(r_{0}^{2}\left(1+\alpha_{1}\right)\right.}{\left(1-3 \alpha_{1}^{2}\right)\left(1-\alpha_{1}\right)} \frac{\left(1-\alpha_{1}\right)^{2}}{\alpha_{0}^{2}} \cdot \frac{3\left(1-\alpha_{1}^{2}\right)}{\left(1-3 \alpha_{1}^{2}\right)}>3
$$

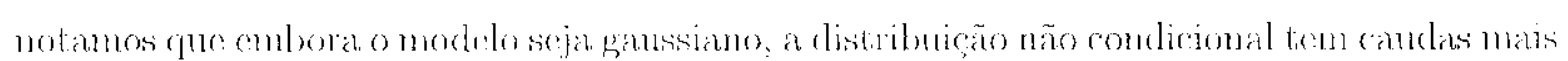

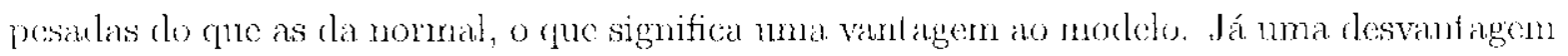

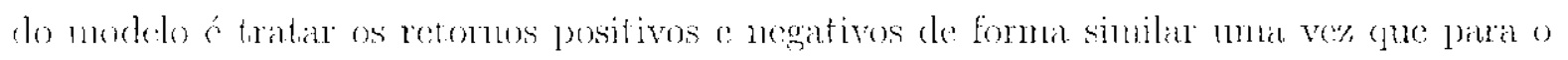
válculo da volatilidude säo neados os quadrados dos retormos

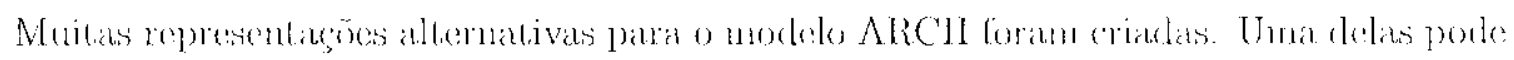
ser obticla faccuto

$$
Z_{l}^{2}=\sigma_{l}^{2} \cdot\left(Z_{i}^{2} \cdots \sigma_{l}^{2}\right)
$$

an que $\sigma_{t} \cdots \alpha_{0}+\sum_{j-1}^{p} \alpha_{j} Z_{i}^{2}, \mathrm{e} Z_{i}=\sigma_{t} \varepsilon_{l}$. Assim

$$
Z_{l}^{2} \cdots \alpha_{t_{0}}+\sum_{j=1}^{p}\left(\gamma_{j} Z_{i \cdot j}^{2}+\left(\left(\sigma_{i} \varepsilon_{i}\right)^{2}-\sigma_{l}\right)=\alpha_{0}+\sum_{j-1}^{p}\left(r_{j} Z_{i}^{2}+\nu_{t},\right.\right.
$$

cun que $\nu_{t}=\sigma_{t}^{2}\left(\varepsilon_{t}^{2}-1\right)$. Portanto temos mu modelo $\mathrm{AR}(\mathrm{p})$ para $Z_{l}^{2}$ com ruído $\nu_{t}$. Através

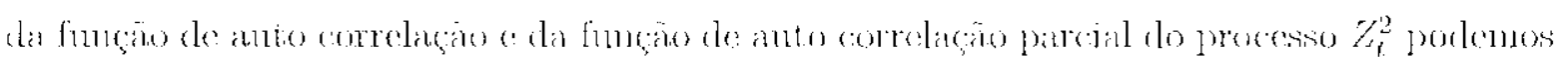

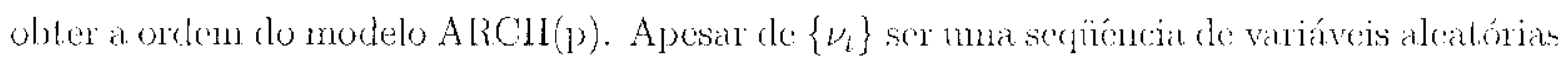

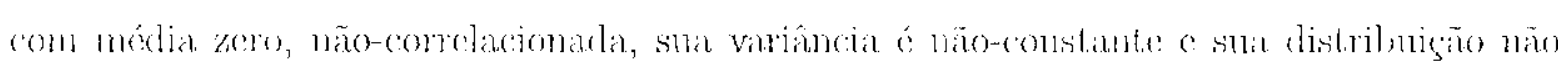

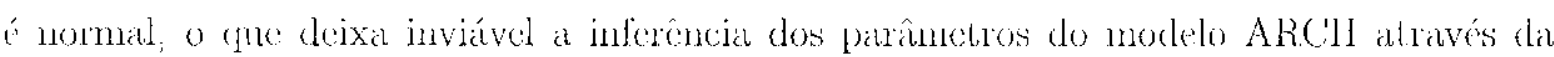
representacion on (B.22)

Vale ressaltar que a distribuigäo leptocúrtion das sóries de retoruos é nellhor calarteri-

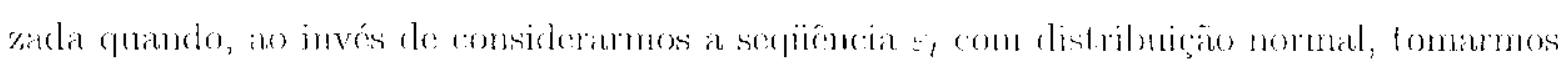
una distribuigão condicional näo normal a $\varepsilon_{t}$. Como por exemplo de algumas distribuiçoes

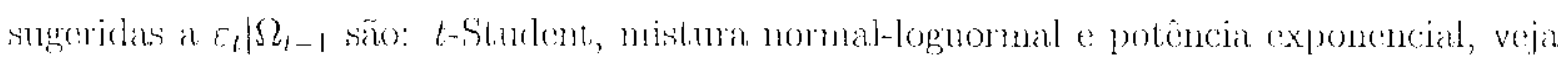
p.e. Bollerslev (1987), Hsioh (1989) e Baillio \& Bollowlov (1989), respectivanente. 


\section{B.2 Modelos GARCH $(p, q)$}

Várias das aplicaçoes do modelo ARCH necessitan de muiton valores passados da série. Unu alternativa mais flexível é dada pelo AlCCll generalizado, GARCH(p,q), modelado por Bollerslev Bollerslev (1986)

$$
\begin{aligned}
& Z_{t}=\sigma_{1} \varepsilon_{1} \\
& \sigma_{l}^{2}=\sigma_{0}+\sum_{i=1}^{p} \alpha_{1} \eta_{t-i}+\sum_{j=1}^{q} \beta_{j} \sigma_{t-j}^{2}
\end{aligned}
$$

con cue $\left\{\varepsilon_{l}, l \geq 0\right\}$ o mala sequirncia i.i.d com $E\left(e_{l}\right)-0$ e $\operatorname{Var}\left(\varepsilon_{l}\right)=1$.

() morlelo (B.23) pole ser escrito como

$$
\sigma_{l}^{2}=\alpha_{0}+\alpha(B) \varepsilon_{t}^{2}+\beta(B) \sigma_{t}^{2}
$$

ent clue $\alpha(B)=a_{1} B+\cdots+\alpha_{p} B^{\prime \prime}, \beta(B)=\beta_{1}+\cdots+\beta_{q} \beta^{\prime}$ e $B$ ć o operador retardo. Para

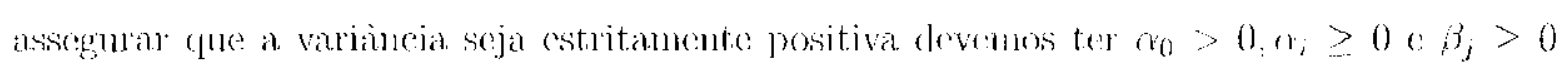
piria $;=1, \ldots, p, j \ldots 1, \ldots, q$.

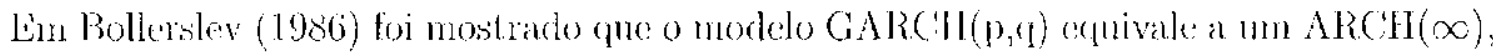
isto é, mu $\Lambda$ RCH de ordem intinila, se as rázes de $1-\beta(B): 0$ estiverem fora do círoulo unitário.

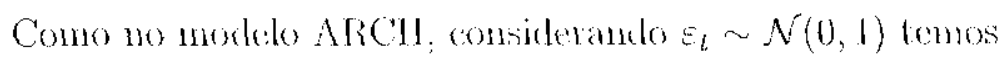

$$
Z_{t} \mid \Omega_{t-1} \sim \mathcal{N}\left(0, \sigma_{i}^{2}\right)
$$

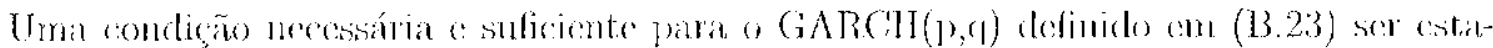
rionánio

$$
\sum_{i=1}^{p} \alpha_{i}+\sum_{j=1}^{\prime \prime} \beta_{j}<1
$$

Terenos entian

$$
\begin{gathered}
\mathrm{E}\left(Z_{i}\right) \therefore 0 \\
\operatorname{Var}\left(Z_{i}\right) \cdots \frac{\alpha_{0}}{1-\left(\sum_{i=1}^{p} \alpha_{i}+\sum_{j=1}^{q} \beta_{j}\right)}
\end{gathered}
$$

$\operatorname{Cov}\left(Z_{i}, Z_{1+k}\right)=0, \quad k \geq 1$ 
Uma ontra representugăo para (B.23) é oblida considerando

$$
\nu_{i}=Z_{i}^{2}-\sigma_{t}^{2}
$$

Substituindo a equaryano (B.31) en (B.23) obtemos

$$
Z_{i}^{2}-\alpha_{0}+\sum_{i=1}^{\eta^{2}}\left(\alpha_{i}+\beta_{i}\right) z_{l-i}^{2}+\nu_{l}-\sum_{j=1}^{q} \beta_{j} \nu_{l-j},
$$

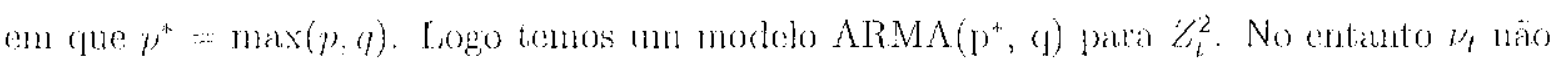

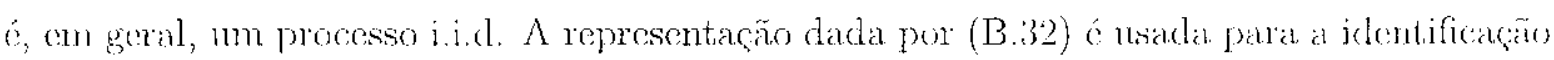
dar orden do morledo.

\section{$\operatorname{GARCH}(1,1)$}

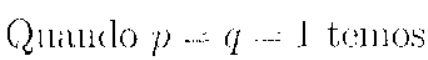

$$
\sigma_{l}^{2}=\alpha_{0}: \alpha_{1} Z_{l}^{2}+\beta_{1} \sigma_{l 1}^{2}, \quad \alpha_{0}>0, \alpha_{1} \geq 0, \beta_{1} \geq 0
$$

Pála que o processo seja estiacionário no senticlo amplo devenus ter $\alpha_{1}+\beta_{\downarrow}<1$. E então terentos

$$
\mathrm{E}\left(Z_{i_{1}}\right)=0 \quad \operatorname{Var}\left(Z_{t}\right)=\frac{\alpha_{0}}{1 \cdots \alpha_{1}-\beta_{1}} .
$$

Vames lanbén analisar a curtose deste processo

$$
K-\frac{B\left(Z_{t}^{\prime}\right)}{\left[\mathrm{E}\left(Z_{i}^{\prime}\right)\right]^{2}=}=\frac{3\left[1-\left(\alpha_{1}+\beta_{1}\right)^{2}\right]}{1-\left(\alpha_{1}+\beta_{1}\right)^{2}-2 \alpha \alpha_{1}^{3}}>3
$$

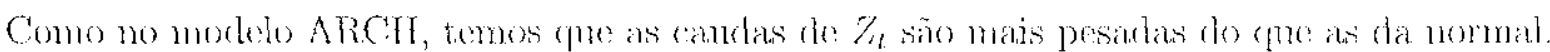

Em geral, identificar a orem de mon modelo GARCH a ser ajustado é clificil. En geral Siro utilizarlos molelos de onden baixa como $(1,1),(1,2)$ on $(2,1)$.

Notson (L990a) mostron que o modelo a tempo discreto GARCL $(1,1)$ converge para nur

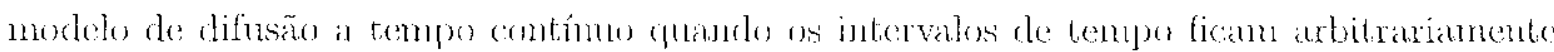
pequenos. Já em Nelson (1992) foi mostrado que se o verdadeiro uodelo é um modelo de

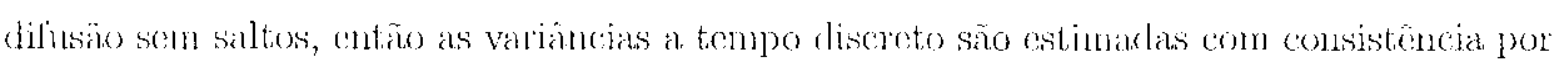

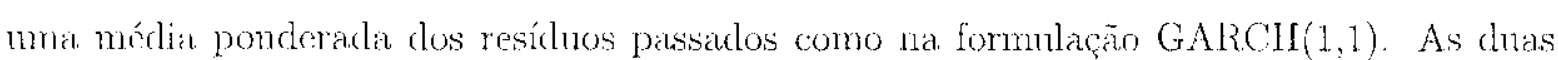

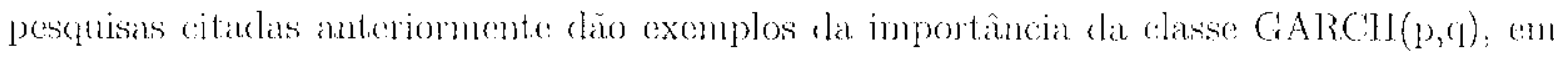
particular do GAIRCH $(1,1)$. 


\section{B.3 Modelos EGARCH $(p, q)$}

Os modclos GARCH(p,q) são capazes de expressar a volatilidade das sérics de retorno das ações. No entanto, os modelos GARCH levam em conta apenas o tamanho dos retomos e não o seu sinal, ou seja, ignoram o fato deles serem positivos ou negativos. Isto sugeriu que se procurasse um modelo no qual $\sigma_{\imath}^{2}$ respondesse assimetricamente ao resíduo positivo e negativo.

O traballıo de Nelson (1991) introcluziu o modelo exponencial GARCH ou EGARCH dado por

$$
\ln \left(\sigma_{t}^{2}\right)=\alpha_{t}+\sum_{k=1}^{\infty} \beta_{k} g\left(\varepsilon_{t-k}\right), \quad \beta_{1}=1,
$$

en que $\alpha_{l, t} \geq 1$ e $\beta_{k}, k \geq 1$ são seqüiencias não estocásticas reais.

Para descrever a relação entro a mulança dr: sinal do rotomo e a volatilidacle existente, a função $g\left(\varepsilon_{l}\right)$ deve ser capaz de expressar a magnitude c o sinal de $Z_{l}$.

Um excmplo importante para a função $g$ é:

$$
g\left(\varepsilon_{l}\right)-\theta \varepsilon_{l}+\gamma\left(\left|\varepsilon_{l}\right|-\mathrm{E}\left(\left|\varepsilon_{l}\right|\right)\right)
$$

Por construção $g\left(\varepsilon_{t}\right)$ é uma seciüencia i.i.d. com nédia zcro. Podemos escrever $(B .37)$ na. forma

$$
g\left(\varepsilon_{l}\right)= \begin{cases}(\theta+\gamma) \varepsilon_{t}-\gamma \mathrm{E}\left(\left|\varepsilon_{l}\right|\right), & \text { se } \varepsilon_{l} \geq 0 \\ (\theta-\gamma) \varepsilon_{t}-\gamma \mathrm{E}\left(\left|\varepsilon_{t}\right|\right), & \text { se } \varepsilon_{t}<0\end{cases}
$$

F isto nos perrrite notar na volatilidade o cliamado "efeito alavanca", ou seja, a resposta da volatilidade é mais rápida a retornos negativos do que a retornos positivos. Logo $g\left(\varepsilon_{1}\right)$ perrnite que o processo da variância condicional $\left\{\sigma_{i}^{2}\right\}$ responda assimetricamente a quedas e aumontos 110 preço dia ação, ou soja, é capaz de cxpressar o "efcito alavanca".

\section{B.4 Modelos ARCH-M}

Muitas teorias em finanças são bascadas na existência de uma relação entre risco e retorno esperado. Vale ressaltar que o risco é muitas vezes aproximada pela volatilidade. No modelo ARCH-M introduzido por Engle et al. (1987) à esperança condicional é uma função explícitia da variância condicional 


$$
\begin{gathered}
Y_{l}=X_{t-1} \beta+g\left(\sigma_{t}^{2}\right)+Z_{t} \\
Z_{\iota}=\sigma_{\iota} \varepsilon_{l} \\
\sigma_{t}^{2}=\alpha_{0}+\sum_{j=1}^{p} \alpha_{j} Z_{t-j}^{2} .
\end{gathered}
$$

Neste modelo um aumento na variância condicional será associado com um aumento ou uma diminuição na esperança condicional de $Y_{l}$ dependendo do sinal da derivada de g com relação a $\sigma_{t}^{2}$. As escolhas mais comuns para $g$ säo funçōes lineares ou logarítmicas de $\sigma_{t}^{2}$ ou $\sigma_{t}$

\section{B.5 Modelos IGARCH}

Chamamos de processo JGARCH um processo GARCII tal cuee, an invés cla equação (B.27) ser satisfeita, tenıs

$$
\sum_{i=1}^{p} \alpha_{i}+\sum_{j=1}^{l} \beta_{j}=1
$$

So $Z_{t}$ segue um processo IGARCH então a variância não condicional de $Z_{t}$ ć infinita, e nem o processo nem seu quadrado $Z_{l}^{2}$ têm covariância estacionária. No entantó ć possível que $Z_{t}$ seja originário de un processo estritanente estacionário $n$ sentide da densiltarle não condicional de $Z_{t}$ ser a mesma para todo $t$, veja Nelson (1990b). 


\section{Referências Bibliográficas}

Anclasen, T. G. (1994). Stochastic antoregressive volatility: A framework tor volatility nodeling. Malhematicul finance, 1, $75-102$.

Andersen, T. G., Bollerslev, T., Diobold, F. X. \& Labys, P. (2001). The clistribution of realized exchange rate volatility. Jounal of The Anerinan Statistical Assoriation, 96 , $12-5,5$.

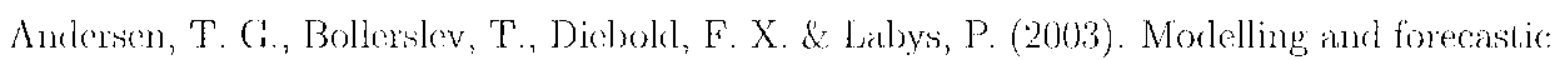
realized volatility. Eronomelnica, 2(71), 579 (i20

Baillie, R. T. \& Bollerstev, T. (1989). The message in daily exchange rates: a conditional variance tale. Joumal of Business and Fconomic Statistics, 7, $297-305$.

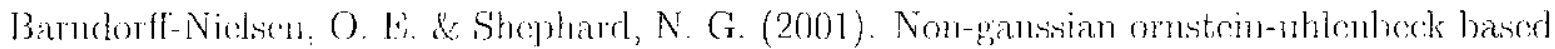
models and some of their uses in linancial econometries. Joumal of The Royal Statistical Soctety, Series B $(63), 167211$.

Blakk, F. \& Scholes, M. S. (1973). 'the pricing of options and corporate liabilitics. Journal of Political Eronomy, 7, 637-51.

Bollerslev, T. (1986). Generalized antoregressive conditional heteroskedasticity. Joumal of Econometrics: $31,307-327$. 
Bollensev, T. (1987). A conditional hoterosketastic time series monlel for speculative prices and rates of return. Reven of Economics and Statistics, 69, 512547.

Bollersilev, 'J', Chou, R. Y. \& Kroner, K. F. (1992). ARCH mosklling in linance. Jonmul os Econometries, 52, $5-59$

Bollerslev, T., Engle, R. F. \& Nelson, D. B. (1994). ARCH nodels. Mandlook of Econometries: IV, 2959 3038. dhapher 19

Caines, P. F. (1988). Lineat Stochastir Systems. Wiloy, New York.

Carnero, A., P'ena, D. \& Ruiz, E. (2004). Persistence and kurtosis in GARCH and stochastic volatility models. Jomnal of Financinl Econometnics, 2, 319-312

Cinlar, E. (1975). Introduction to stochastic processes. Prentice-Hall, London.

Davis, M. IT. A. \& Vinter, R. B. (1985). Stochastic Molelling and Control. Chapman d Itall, Lomiclon New York

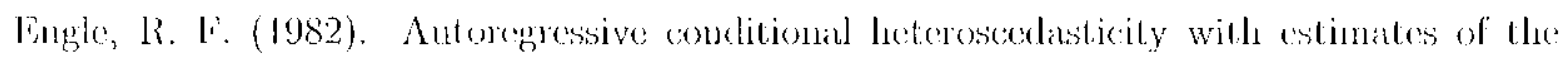
variance os United Kingdom inflation. Fconometrica, 50(1), 987-1007.

Engle, R. F. \& Bollerslow, T. (1986). Nodelling the persistence of conditional variances Fronometric Reviews, 5, 150 .

Fingle, R. R., T.ilion, D. M. \& Robins, R. P. (1987). Estinating time varying tisk premia in the term structure: the ARCH M nodel. Econometra, 55(2), 391-407.

Fania, E. F. (1965). The behavion of stock market prices. Journal of Business, 38, 34-105.

Fomnier, D. (2000). AD Model Builder Version 5.0.1. Otter Rosearch Ltal, Canada.

Gameman, D. (1997). Markov Cham Monte Carto, Stochastic Simulation for Bayestan Inference. Chapman \& Hall, Iondon

Goland, A. L. G. \& Smith, A. F. M. (1995). Sampling-based approaches to calculating marginal densitics. Joumal of the Amerikn Statistical Association, 85, 398-109 
Gelman, A. \& Bubin, D. (1992). Tuference from iterative simulation using multiple sequences. Statistical Sclicuce, $7,157-511$.

Geweke, J. (1992). Evaluating the accuracy of sampling-based approaches to the calculation of posterion moments. Bayesian Statistics, 4, 169- 193

Ghyscls, E., Harvey, A. \& Renault, E. (1995). Stochastic volatility. Handbook of Statistics, 14.

Griewank, A. \& Corliss, G. L'. (1991). Automatic Differentiation of Algorithms: Theory, Implementalion and Applation. SIAM, Philatlelphia.

Hamilton, J. D. (1994). Thme Series Analysis. Princeton University Press, Princeton.

Harvey, A. C. (1989). Forecasting Structural Time Series Models and the Kalman Filter. Camburige? Univensity Press, New York.

Ianvey, A. C. \& Shephatel, N. G. (1996). Estination of an asynmetric stochnstic volatility

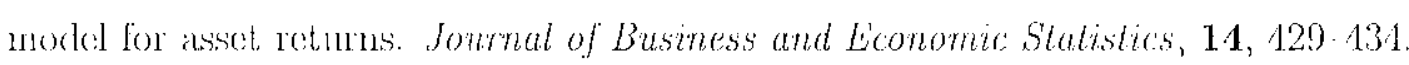

Harvey, A. C., liniz, L. \& Shephard, N. (1994). Multivariate stochastic variance models. Revew of ticonontic Studies, $61,217264$.

Hastings, W. K. (1970). Monte Carlo sampling methods using Markov chains and their application. Bionetrika, 57, 217-261.

Hsich, D. A. (1989). Morlelling leterosedasticity in claily foreing exchange rates. Joumal of Bussiness und Economic Statistics, 7, 307 317.

Hull, J. (1996). Introducho nos menados futuros e de opcós. Bolsa de Mercados \& Futnros second edition.

ITull, J. \&, White, A. (1987). 'The prineing of options on asseds with stochastic volatilities Joumal of Finance, 42, 281 300 .

Issler, J. V. (1999). Estimatiug and forecasting the volatility of brasilian finance. Revista de Econometria $19(1)$ 
Jacquice, H., Polson, N. (: \& Rossi, P. L. (2002). Bayesian analysis of stochastic volatility models with fat-tails and correleted errors. Jommal of Econometrics.

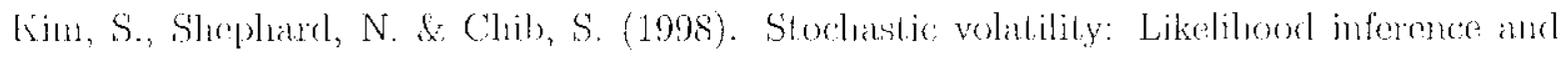
comparison will ARCH nodels. Review of Leonometrics Studies, 85, 361393.

Koopman, S. J. \& Uspenky, F. H. (2002). The stodhatic volatility in nucan model: Empirical evidence from international stock markets. Sommal of Applied. Econometrics, 17(6), 667 689.

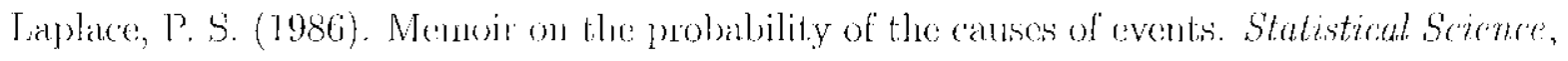
$1,364-378$.

Lee, 'T. K. Y. S Tse, Y. K. (1991). 'Tem stmeture of interest rates in singapore astan dollat market. Joumal of Applind Econometrics, 6, 113152

Metropolis, N., Rosenbluth, A. W., Tellex, A. H. \& Tellex, F. (1953). Erplations of state

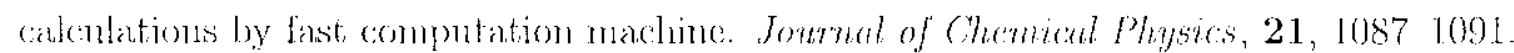

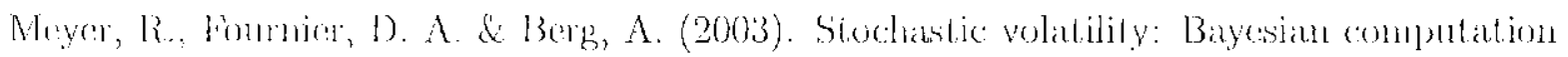
nsing antonatio differentiation and the extended Kahman filter. Ecometrics Journal, 6 , $108 \cdots 120$

Nilne, W. E. (1953), Numerion Solution of Differential Equations. Wilcy, Sow York.

Morettin, P. A. \& Toloi, C. M. C. (2001). Analise de Sertes Temporais. Projeto Fisher.

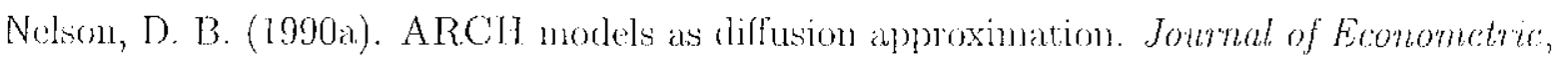
45, $7-38$

Netson, D. B. (1990h)). Stationatity and persistence in the GARCH(1,1) model. Econometrit: Thery, 6, 318331

Nobon, D. B. (1991). Conditional heteroskecedasticity in asset returns: A new approach. Liconometrica, $59(2), 317370$.

Nelsom, D. B. (1992). Fillering and forecasting with missuerifoel ARCH mudels i: Getting the right variance with the wong model. Journal of Exonometrics, 52, 61.90. 
Oksendal, B. (1998). Stochastio Differtntial Equations: An Introduction with Aplicalions. Springer, fifth edition.

Polegrín, C. 13. (2001). Fistimation de Modelos de Volatiladad Estocástica y Modelos de Conponentes Inobsenudas Condacionalmente Hetroceduslicos. Ph.D. thesis, Universidad Carlos IIr de Madrid.

Press, S. J. (1989). Bayesian Statisties: Principles, Models, and Applications. John Wiley Sons.

Ripley, B. D. (1987). Storhastic Sinulation. Wiley, New York.

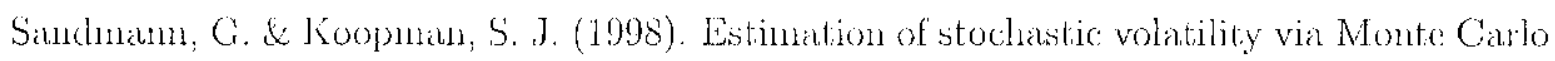
maximmun likelihood. Jommal of Eromometrics, 87, 271301.

Sen, P. K. \& Singer; J. M. (1993). Large Sample Methods in Statistics: An Introduction with Applications. Chaljmman \& Hall, New York.

'Taylor, J. B. (1982). Finducial returns modelled by the product of two stochastic processes, al stuly of daily sugar prices. Time Sertes Analysis: 'Theory and Practice, 1, 203-226.

Taylor, S. (1994). Mockelling stochastic volatility: A review and conparative study. Mathematical Finance, 4, 183-204.

Taylor, S. J. (1986). Modelmo Fincheial Time Series. Clichester.

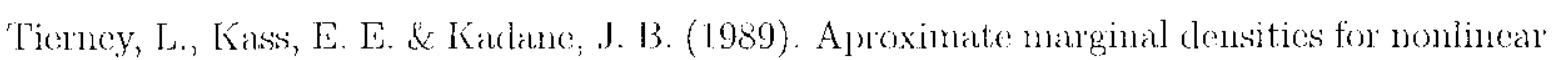
functions. Biometrika, 76,425433 .

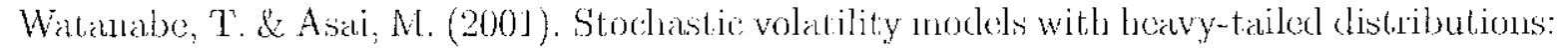

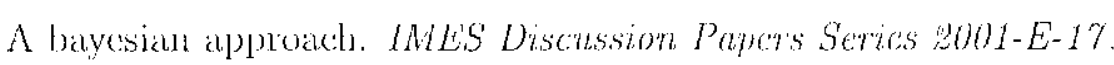

